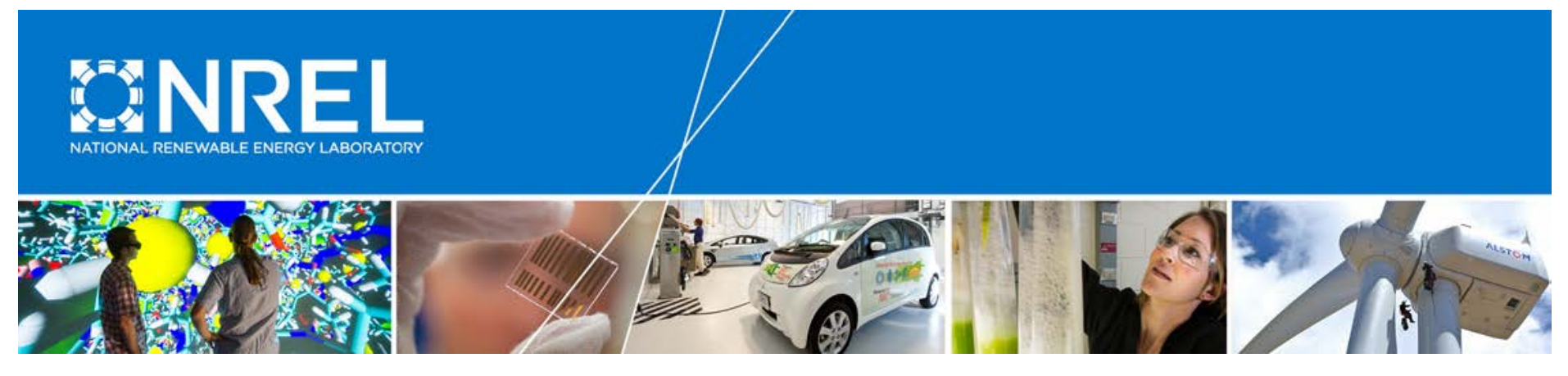

\title{
Photovoltaic Impact Assessment of Smart Inverter Volt-VAR Control on Distribution System Conservation Voltage Reduction and Power Quality
}

Fei Ding, Adarsh Nagarajan, Sudipta Chakraborty, and Murali Baggu

National Renewable Energy Laboratory

Andu Nguyen, Sarah Walinga, Michael McCarty, and Frances Bell SolarCity

NREL is a national laboratory of the U.S. Department of Energy Office of Energy Efficiency \& Renewable Energy Operated by the Alliance for Sustainable Energy, LLC

This report is available at no cost from the National Renewable Energy Laboratory (NREL) at www.nrel.gov/publications.

Technical Report

NREL/TP-5D00-67296

December 2016 


\section{Photovoltaic Impact Assessment of Smart Inverter Volt-VAR Control on Distribution System Conservation Voltage Reduction and Power Quality}

Fei Ding, Adarsh Nagarajan, Sudipta Chakraborty, and Murali Baggu

National Renewable Energy Laboratory

Andu Nguyen, Sarah Walinga, Michael McCarty, and Frances Bell SolarCity

Prepared under Task No(s). ST13IN35, WRGE1001
National Renewable Energy Laboratory 15013 Denver West Parkway Golden, CO 80401

303-275-3000 • www.nrel.gov
NREL is a national laboratory of the U.S. Department of Energy Office of Energy Efficiency \& Renewable Energy Operated by the Alliance for Sustainable Energy, LLC

This report is available at no cost from the National Renewable Energy Laboratory (NREL) at www.nrel.gov/publications.

\section{Technical Report}

NREL/TP-5D00-67296

December 2016

Contract No. DE-AC36-08GO28308 


\section{NOTICE}

This report was prepared as an account of work sponsored by an agency of the United States government. Neither the United States government nor any agency thereof, nor any of their employees, makes any warranty, express or implied, or assumes any legal liability or responsibility for the accuracy, completeness, or usefulness of any information, apparatus, product, or process disclosed, or represents that its use would not infringe privately owned rights. Reference herein to any specific commercial product, process, or service by trade name, trademark, manufacturer, or otherwise does not necessarily constitute or imply its endorsement, recommendation, or favoring by the United States government or any agency thereof. The views and opinions of authors expressed herein do not necessarily state or reflect those of the United States government or any agency thereof.

This report is available at no cost from the National Renewable Energy Laboratory (NREL) at www.nrel.gov/publications.

Available electronically at SciTech Connect http:/www.osti.gov/scitech

Available for a processing fee to U.S. Department of Energy and its contractors, in paper, from:

U.S. Department of Energy

Office of Scientific and Technical Information

P.O. Box 62

Oak Ridge, TN 37831-0062

OSTI http://www.osti.gov

Phone: 865.576.8401

Fax: 865.576.5728

Email: reports@osti.gov

Available for sale to the public, in paper, from:

U.S. Department of Commerce

National Technical Information Service

5301 Shawnee Road

Alexandria, VA 22312

NTIS http://www.ntis.gov

Phone: 800.553 .6847 or 703.605 .6000

Fax: 703.605.6900

Email: orders@ntis.gov 


\section{Acknowledgments}

This work was funded under a cooperative research and development agreement (CRD-14-563) between the National Renewable Energy Laboratory (NREL) and SolarCity. NREL thanks SolarCity and the U.S. Department of Energy Solar Energy Technologies Office for cost-sharing this work. The authors from SolarCity also thankfully acknowledge the financial and technical support they received from the Hawaiian Electric Companies (HECO).

The authors thank HECO and Pacific Gas and Electric Company (PG\&E) for providing the feeder models and data. We also appreciate the knowledgeable input and helpful feedback provided by the following reviewers, which greatly improved this report: Barry Mather (NREL), Julieta Giraldez (NREL), Ryan Hanley (SolarCity), Marc Asano (HECO), Reid Ueda (HECO), Thomas Russell (PG\&E), Andrew Hanson (Accenture). 


\section{List of Acronyms}

$\mathrm{AC}$

ANSI

COM

CVR

DC

EPRI

HECO

IEC

LTC

NRECA

NREL

PG\&E

PNNL

PQS

PV

QSTS

RVC

SAVFI

SAVUI

SAVMVI

SCDOI

SELI

SI

SRPDI

VAR

$\mathrm{VO}$

VVC

RMS
Alternative current

American National Standards Institute

Component object interface

Conservation voltage reduction

Direct current

Electric Power Research Institute

Hawaiian Electric Companies

International Electrotechnical Commission

Load tap changer

National Rural Electric Cooperative Association

National Renewable Energy Laboratory

Pacific Gas and Electric Company

Pacific Northwest National Laboratory

Power quality score

Photovoltaic

Quasi-static time series

Rapid voltage change

System average voltage fluctuation index

System average voltage unbalance index

System average voltage magnitude violation index

System control device operation index

System energy loss index

Smart inverter

System reactive power demand index

Volt ampere reactive

Voltage optimization

volt-VAR curve

Root mean square 


\section{Executive Summary}

This report presents an impact assessment study of distributed photovoltaic (PV) systems with smart inverter volt-VAR control on voltage reduction energy savings and distribution system power quality. Conservation Voltage Reduction (CVR) can enable voltage reduction energy savings through traditional CVR or Voltage Optimization (VO). Traditional CVR implementations have relied on deployment of local device control logic that ensures operation of load tap changers and voltage regulators such that customer voltages are maintained within lower target band of the acceptable delivery voltage range. The advent of voltage optimization (VO) systems providing centralized control of load tap changers, voltage regulators, and capacitor banks has improved upon the voltage reduction achievable with traditional CVR implementations. These improvements in traditional CVR are still limited by their top down approach to controlling voltage. Increased penetrations of PV on distribution systems equipped with smart inverters provide a new opportunity to control and optimize local voltage by regulating the reactive power output. These new opportunities increase the optionality of voltage optimization to potentially increase CVR energy savings. This report proposes a methodology to implement an optimized voltage reduction scheme by operating voltage regulators, capacitors, and autonomous smart inverter volt-VAR control to achieve an increased voltage reduction benefit relative to traditional CVR implementations.

Power quality is an important consideration when operating a distribution system, especially when implementing CVR. It is easy to measure the individual components that comprise power quality, but a comprehensive method to incorporate all of these values into a single score has yet to be undertaken. As a result, this report proposes a power quality scoring mechanism to measure the relative power quality of distribution systems using a single number, which is aptly called the "power quality score" (PQS).

Both the voltage reduction and PQS methodologies were applied to two distribution system models: one obtained from the Hawaiian Electric Companies (HECO) and another obtained from Pacific Gas and Electric Company (PG\&E). These two models were converted to the OpenDSS platform using previous model conversion tools that were developed by the National Renewable Energy Laboratory. Multiple scenarios including various PV penetration levels and smart inverter densities were simulated to analyze the impact of smart inverter volt-VAR support on voltage reduction energy savings and feeder power quality. To analyze the additional voltage reduction benefit and PQS, an annual simulation was conducted for each scenario.

The key findings from this report include:

- Without PV, voltage reduction energy savings of $1.51 \%$ and $3.86 \%$ were achieved for the HECO and PG\&E distribution system models, respectively. In some cases, randomly distributed PV without smart inverters still increased voltage reduction energy savings.

- Voltage reduction energy savings increased with autonomous smart inverter volt-VAR control. Compared to the no-PV base case, the HECO system saw a $1.37 \%$ increase in voltage reduction energy savings with less than $150 \%$ PV penetration $(150 \%$ of peak loads) and $100 \%$ smart inverter density. The PG\&E system saw a $0.44 \%$ increase in voltage reduction energy savings with less than $100 \%$ PV penetration and $100 \%$ smart inverter density. 
- Without VO voltage reduction measures in place, smart inverters generally had an overall positive impact on the PQS. With VO voltage reduction measures in place, because VO voltage reduction energy savings were prioritized over the PQS, the implementation of the VO scheme lowered certain power quality scoring metrics, causing an overall lower PQS.

The report concludes with a detailed discussion of takeaways and next steps for future research. All codes developed in this report are available for utilities and other parties to improve upon and perform similar studies with proper permissions. 


\section{Table of Contents}

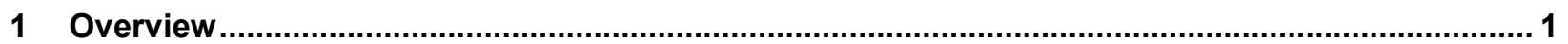

2 Conservation Voltage Reduction and Voltage Optimization ........................................................ 3

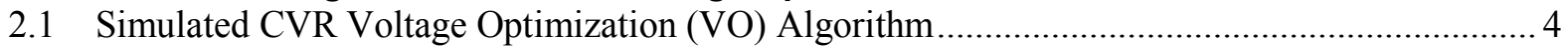

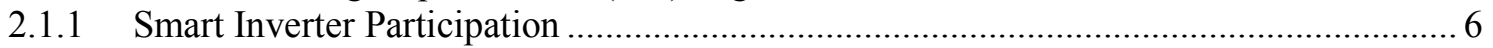

2.1.2 Demonstration of CVR Voltage Optimization Methodology ..................................... 10

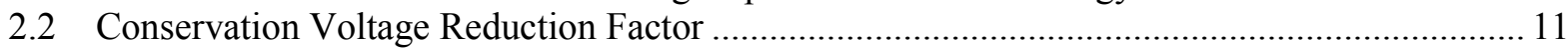

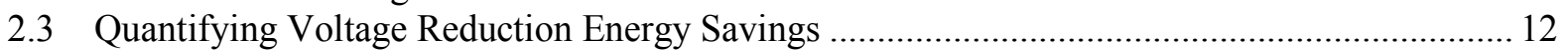

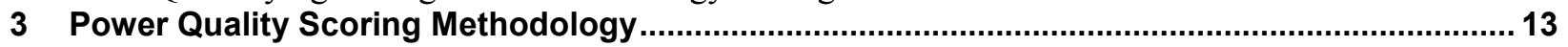

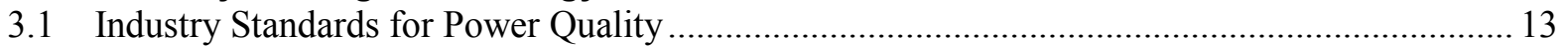

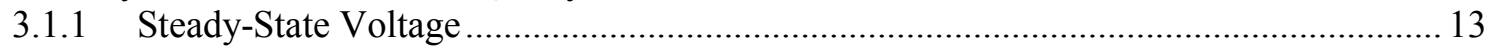

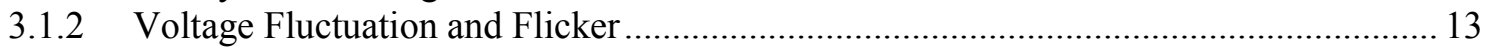

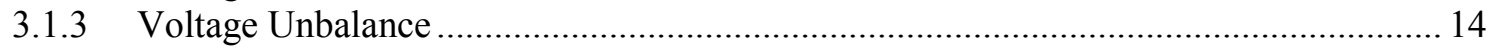

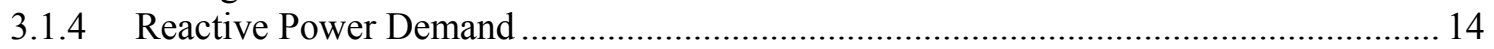

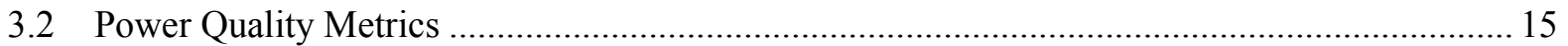

3.2.1 Metric 1: System Average Voltage Magnitude Violation Index ................................... 15

3.2.2 Metric 2: System Average Voltage Fluctuation Index .................................................. 16

3.2.3 Metric 3: System Average Voltage Unbalance Index ................................................ 17

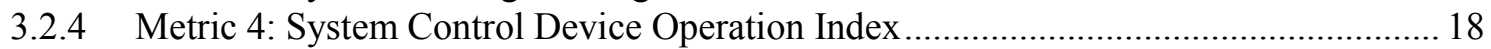

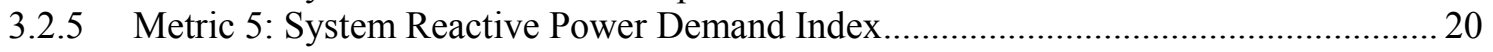

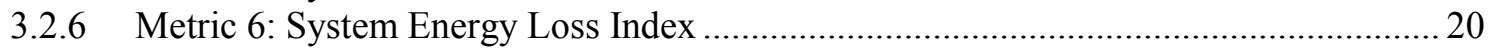

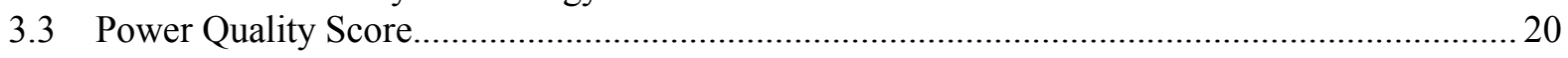

3.3.1 Step 1: Determine Individual Power Quality Metrics .............................................. 21

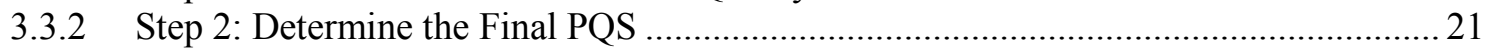

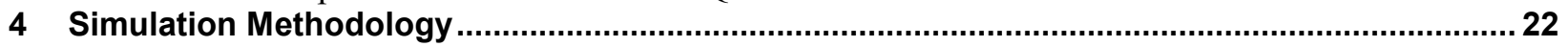

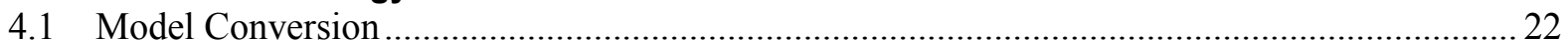

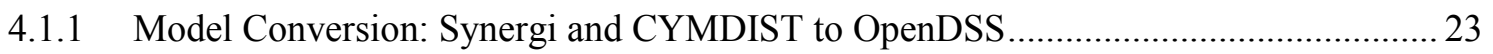

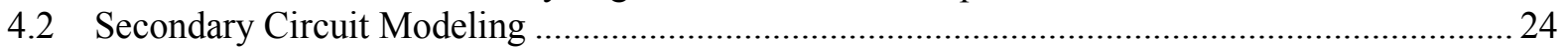

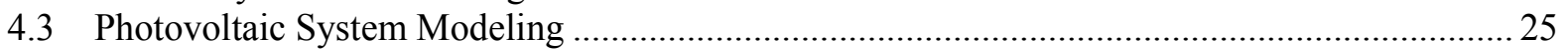

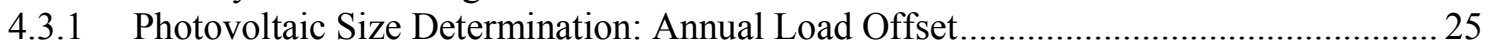

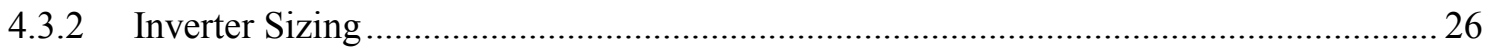

4.3.3 Photovoltaic and Smart Inverter Random Allocation ................................................ 27

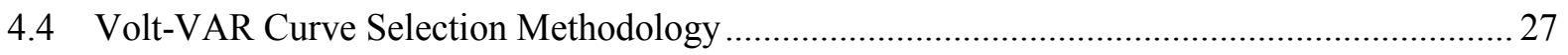

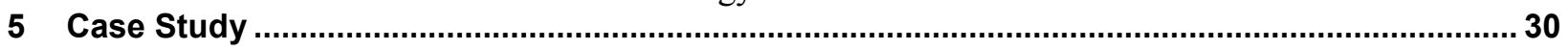

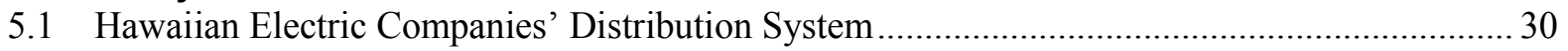

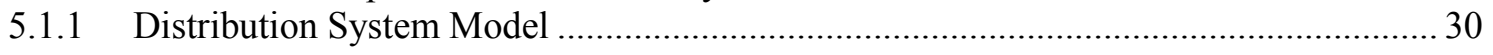

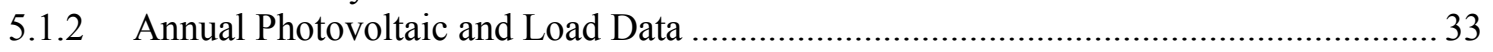

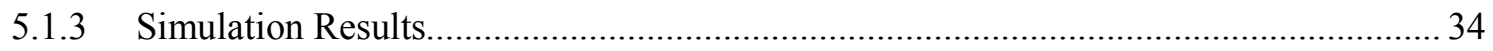

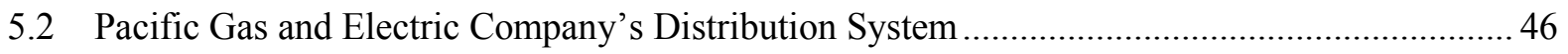

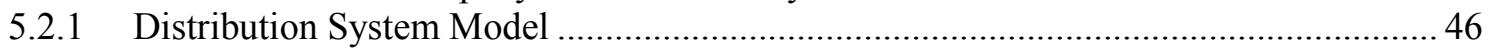

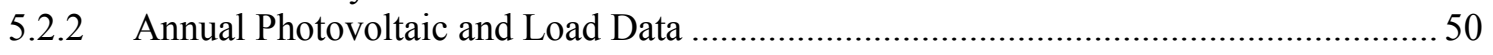

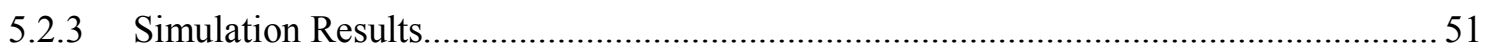

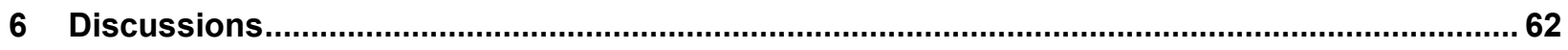

6.1 Normalizing and Weighting of Power Quality Metrics ...................................................... 62

6.2 Distribution Systems Comparison: Hawaiian Electric Companies and Pacific Gas and Electric

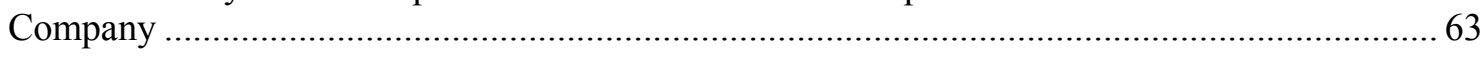

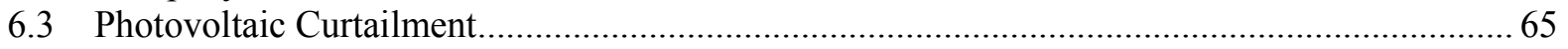

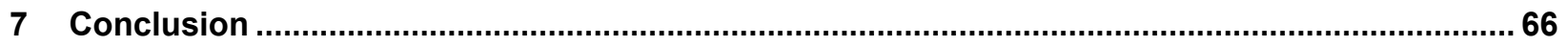

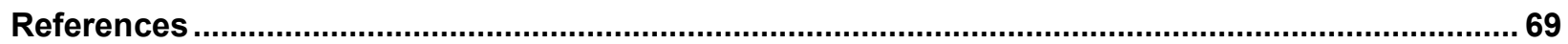




\section{List of Figures}

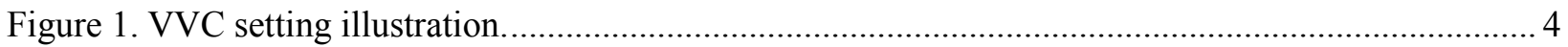

Figure 2. Optimization algorithms for capacitors and substation LTC ................................................. 5

Figure 3. Smart inverter volt-VAR control output algorithm ......................................................... 7

Figure 4. Local SI control: CVR VO algorithm with smart inverters ................................................. 9

Figure 5. Voltage profiles for the PG\&E distribution system on a winter day demonstrating the various

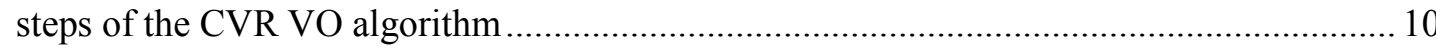

Figure 6. Voltage profiles for the PG\&E distribution system on a summer day demonstrating the various

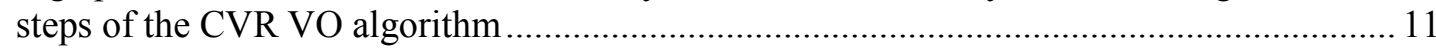

Figure 7. Illustrative QSTS result for steady-state voltage magnitude ............................................... 15

Figure 8. Linear correlation between power quality metric value and its individual score ..................... 21

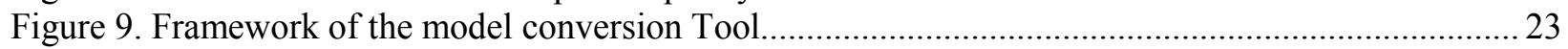

Figure 10. Diagrammatic view of Synergi to OpenDSS model conversion depicting the syntax

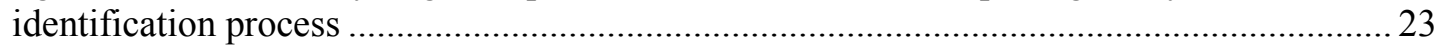

Figure 11. Diagrammatic view of Synergi to OpenDSS secondary addition depicting the syntax

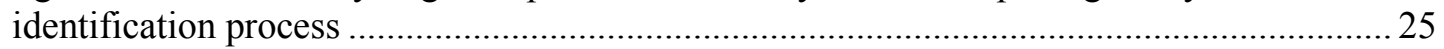

Figure 12. Diagram depicting inverter capacity with reactive power capability range ........................... 26

Figure 13. VVC settings with vvcWidth, vvcCenter, and vvcDeadbandWidth......................................28

Figure 14. Geographical topology view of the HECO distribution system in Synergi and OpenDSS ....... 31

Figure 15. Voltage profile and voltage error of the HECO distribution system ...................................... 32

Figure 16. Positive sequence impedance profile and sequence impedance error of the HECO distribution

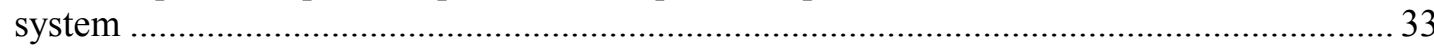

Figure 17. Example illustrating how native load was calculated............................................................ 34

Figure 18. Hourly load demand for the HECO distribution system base case......................................... 35

Figure 19. Annual maximum and minimum voltages for the HECO system base case and CVR VO

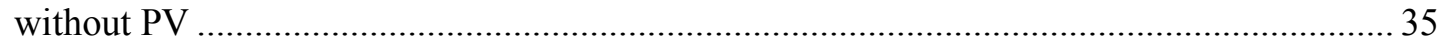

Figure 20. Voltage profiles at peak and minimum load for base case and CVR VO without PV for the

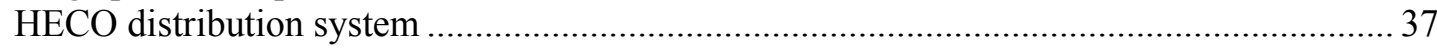

Figure 21. VVC selected to achieve the max voltage reduction energy savings on the HECO distribution

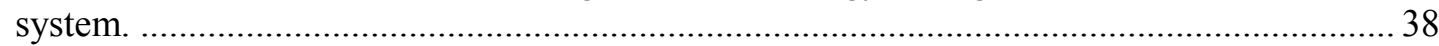

Figure 22. Voltage results for different PV penetrations and smart inverter densities for HECO distribution system.

Figure 23. Voltage reduction energy savings as a function of smart inverter density at different PV penetrations for the HECO distribution system

Figure 24. Voltage reduction energy savings as a function of PV penetration at different smart inverter

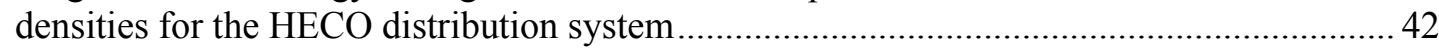

Figure 25. PQS results with different VVC centers for the HECO distribution system .......................... 45

Figure 26. Geographical topology view of the PG\&E distribution system in CYMDIST and OpenDSS.. 48

Figure 27. Current profile and current error of the PG\&E distribution system ...................................... 49

Figure 28. Positive sequence impedance profile and sequence impedance error of the PG\&E distribution

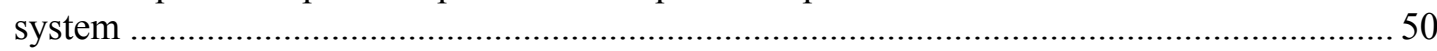

Figure 29. VVC selected to achieve the max voltage reduction energy savings on the PG\&E distribution

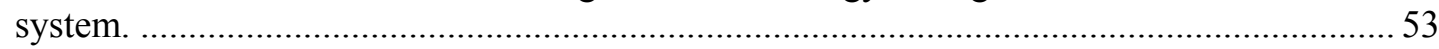

Figure 32. PQS results with different VVC centers for the PG\&E distribution system .......................... 59

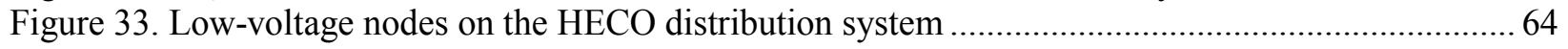




\section{List of Tables}

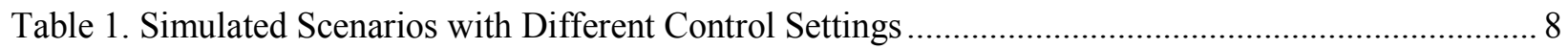

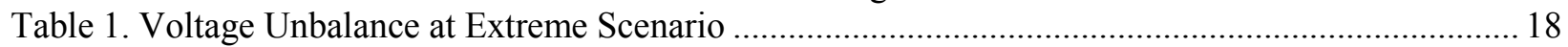

Table 2. Number of LTC Switching Operations in Various Fields of Application [16].......................... 19

Table 4. HECO Distribution System Characteristics ............................................................................. 30

Table 5. Annual PV and Load Data Summary for the HECO Distribution System ................................. 34

Table 6. Power Quality Scores for Base Case and CVR VO without PV for the HECO Distribution

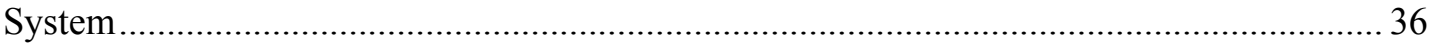

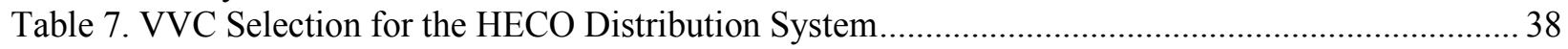

Table 8. Voltage Results by PV Penetration and Smart Inverter Density for the HECO Distribution

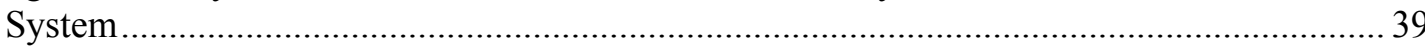

Table 9. Voltage Reduction Energy Savings by PV Penetration and Smart Inverter Density for the HECO

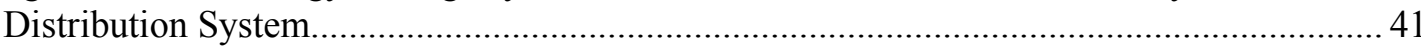

Table 10. Power Quality Score Raw Values by PV Penetration and Smart Inverter Density for the HECO

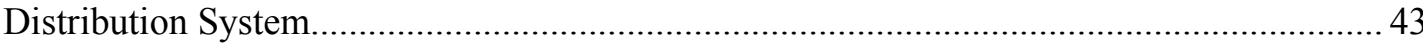

Table 11. Power Quality Score by PV Penetration and Smart Inverter Density for the HECO Distribution

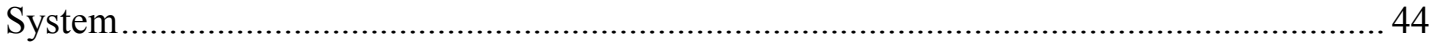

Table 12. Power Quality Score Summary for the HECO Distribution System ...................................... 44

Table 13. Power Quality Score Raw Values without CVR VO for the HECO Distribution System ......... 46

Table 14. Power Quality Score without CVR VO for the HECO Distribution System.............................46

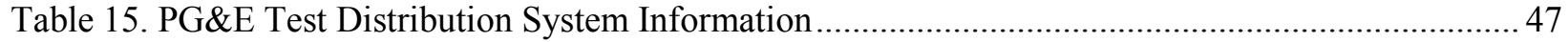

Table 16. Customer Information for PG\&E System Model ................................................................... 47

Table 17. Annual PV and Load Data Summary for the PG\&E Distribution System................................ 51

Table 18. PG\&E Power Quality Scores for Base Case and CVR VO without PV .................................55

Table 19. PG\&E Distribution System's VVC Selection..........................................................................5 52

Table 20. Voltage Results by PV Penetration and Smart Inverter Density for the PG\&E Distribution

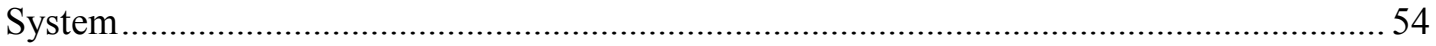

Table 21. Voltage Reduction Energy Savings by PV Penetration and Smart Inverter Density for the

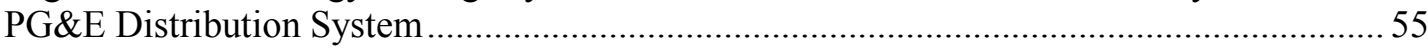

Table 22. Power Quality Score Raw Values by PV Penetration and Smart Inverter Density for the PG\&E

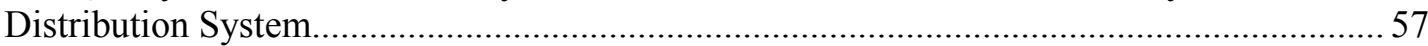

Table 23. Power Quality Score by PV Penetration and Smart Inverter Density for the PG\&E Distribution

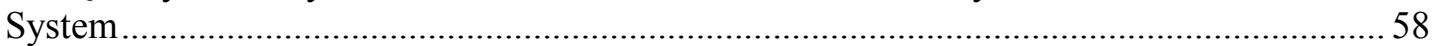

Table 24. Power Quality Score Summary for the PG\&E Distribution System....................................... 58

Table 25. Power Quality Score Raw Values without CVR VO for the PG\&E Distribution System.......... 60

Table 26. Power Quality Score without CVR VO for the PG\&E Distribution System ............................. 61

Table 27. Power Quality Score Summary without CVR VO for the PG\&E Distribution System ............. 61

Table 28. Percentage of PV Curtailment with CVR VO for the PG\&E Distribution System .................... 65

Table 29. Percentage of PV Curtailment with CVR VO for the HECO Distribution System .................... 65

Table 30. Summary of the Voltage Reduction Energy Savings from the HECO and PG\&E Case

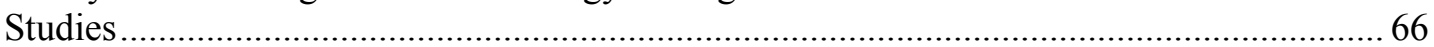

Table 31. Summary of the PQS from the HECO and PG\&E Case Studies without CVR VO .................. 66 


\section{Overview}

As distributed energy resources are becoming more prevalent on today's electric grid, there is increasing opportunity for these resources to support and improve grid operations and efficiency. Traditionally, distributed solar photovoltaics (PV) systems were installed with standard inverters that only output active power. Recently, however, $\mathrm{PV}$ is increasingly being paired with smart inverters that can also supply or absorb reactive power. With this ability to provide reactive power, distributed PV has the potential to support and actively regulate local voltage and power factor on the grid. This local smart inverter control can be done through various smart inverter modes, which include fixed power factor configuration or autonomously controlling the reactive power output based on the local voltage.

One approach that is becoming more common for utilities to improve energy efficiency savings is voltage reduction associated with traditional conservation voltage reduction (CVR) programs and/or voltage optimization (VO) programs. Traditional CVR programs utilize local system control components (load tap changers, line regulators and capacitor banks) to maintain voltage in the lower portion of the acceptable range. Newer Voltage Optimization (VO) schemes effectively flatten the voltage profile and lower voltage, often providing greater overall voltage reduction capability. VO schemes typically leverage voltage control devices that are operated based on central control schemes to optimize voltage levels on circuit. Previous research has shown that controlling local voltage at the secondary level is paramount to maximizing the benefits of voltage reduction programs [1],[2]. Because of this need to control the secondary voltage, distributed PV paired with smart inverters can be utilized to actively regulate local voltage in order to strengthen a utility CVR program. In addition to reducing energy consumption, distributed PV with smart inverters has the ability to improve grid operations and power quality [2],[4].

While it may be intuitive to a utility engineer that this local smart inverter control can improve grid operations, efficiency, and power quality, modeling the potential benefits can seem like a daunting task. This is due to the need for time series circuit models, detailed loading data, accurate PV models, and a simulation environment proficient enough to simultaneously control all of these dynamic components. While several open-source tools, like OpenDSS and GridLABD, can perform complex simulations with smart inverters or CVR [4],[5], these tools have not been designed to handle and control the dynamic interaction between distributed smart inverters and a CVR scheme. Other tools, such as PSCAD or PSLF, that are capable of these complex simulations are not available via open-source. This project aims to build on these open-source tools to provide an integral component for planning and integration of distributed PV into CVR schemes. This is done by:

1. Providing an easy-to-use tool for modeling the impact of distributed PV with smart inverters in a utility voltage reduction scheme.

2. Developing a methodology to help utilities and grid planners identify the impact of PV systems on power quality effectively and efficiently through a power quality scoring mechanism.

3. Demonstrating the methodology by implementing it on two distribution systems from two different utilities. 
As mentioned previously, in addition to measuring the impact to grid efficiency and voltage reduction programs, this report also aims to assess the power quality of a distribution system. The individual components that make up power quality, such as voltage magnitude and unbalance, can be measured in simulations or in the field. However, a comprehensive method to incorporate all of these values into a single score has not been undertaken. This report includes a methodology to quantify this power quality, referred to in this report as the power quality score (PQS). Similar to measuring voltage reduction benefits, measuring the PQS also requires extensive time-series modeling and this report and corresponding tool aim to simplify that modeling process.

Both the voltage reduction evaluation and PQS methodologies were applied to two distribution system models provided by the Hawaiian Electric Company (HECO) and Pacific Gas and Electric (PG\&E). The different utility models provide two different voltage levels and peak loads to analyze the impacts of distributed PV with smart inverters. Additionally, the use of different utility models helps to ensure that the methodology and results of the study can be utilized independent of a specific utility. With these models, an annual simulation of a voltage reduction program was run with varying levels of PV penetration and number of smart inverters. The smart inverters actively regulated voltage at their terminals through an autonomous volt-VAR curve (VVC). Voltage reduction savings and PQS for different scenarios were obtained to assess the impact of smart inverter volt-VAR support on voltage reduction and power quality in distribution feeders.

The code developed for the purpose of the analysis in this study will be available as an open source tool for other interested parties who wish to perform similar analyses on their distribution system models with proper permission. 


\section{Conservation Voltage Reduction and Voltage Optimization}

Utilities can control their distribution system voltage profiles by controlling load tap changers (LTCs), voltage regulators, and shunt capacitors within the substation and distribution system. Using this voltage regulation equipment, a traditional CVR program can be implemented to maintain voltage in the lower portion of the acceptable range reducing demand and energy use from voltage dependent loads. Similarly, voltage optimization (VO) schemes can be implemented to flatten the voltage profile, conserve energy, and reduce peak load. The range of allowable voltages that utility customers can receive is set by the American National Standards Institute (ANSI) in the C84.1 ANSI Standard [6]. This standard defines the electrical utility's responsibility to provide a service voltage (ANSI Range A) between $114 \mathrm{~V}$ and $126 \mathrm{~V}$ on a 120 $\mathrm{V}$ base; in per unit values, this equivalent to 0.95 per unit (pu) and $1.05 \mathrm{pu}$, respectively. Typically, the distribution system voltage decreases as the distance from the upstream voltage regulating equipment increases. During heavy loading, the voltage on the feeder drops the most. In practice, the lower voltages typically only occur at times near peak load and for only a small subset of hours and customers. It has been shown that a $1 \%$ reduction on distribution service voltage can drive a $0.3 \%$ to $1 \%$ load reduction [7] and, as a consequence of the practice of providing a higher than necessary voltage to all customers, there exists an opportunity to improve grid efficiency.

Traditionally, controlling and lowering the distribution voltage has been achieved by optimizing capacitor operation and lowering the output voltage of LTCs and voltage regulators. In recent years, many studies have examined voltage reduction impacts; including those by the Electric Power Research Institute (EPRI) [8] and Pacific Northwest National Laboratory (PNNL) [5]. EPRI's study quantified losses on the distribution system and found that voltage reduction "produces the majority of savings" in energy consumption. PNNL's report found that typical savings from a voltage reduction scheme range from $0.5 \%$ to $4 \%$ of energy consumption and peak reduction on individual distribution circuits.

These studies have largely not included the impact of distributed energy resources (DERs) because DERs have not previously been allowed to participate in voltage regulation. Recently however, an amendment was made to the major DER interconnection standard (IEEE 1547a [9]) in 2014 to allow inverter-based generation to also participate in distribution feeder voltage regulation. By adding distributed PV with smart inverters at various locations on distribution circuit, an opportunity exists to regulate the secondary voltages and, in aggregate, the primary voltage in a way not previously possible when only controlling primary distribution voltage control equipment. Using autonomous volt-VAR control on the smart inverters, the distribution system voltage profile can be further flattened and reduced. This is due to the smart inverter's VVC controlling reactive power output to attempt to bring its terminal voltage closer to the curve's center.

A diagram of a VVC is shown in Figure 1. Each smart inverter with this setting will read its local voltage and evaluate the amount of reactive power to inject or absorb following this curve. By injecting reactive power, the voltage will rise. By absorbing reactive power, the voltage will be lower. 


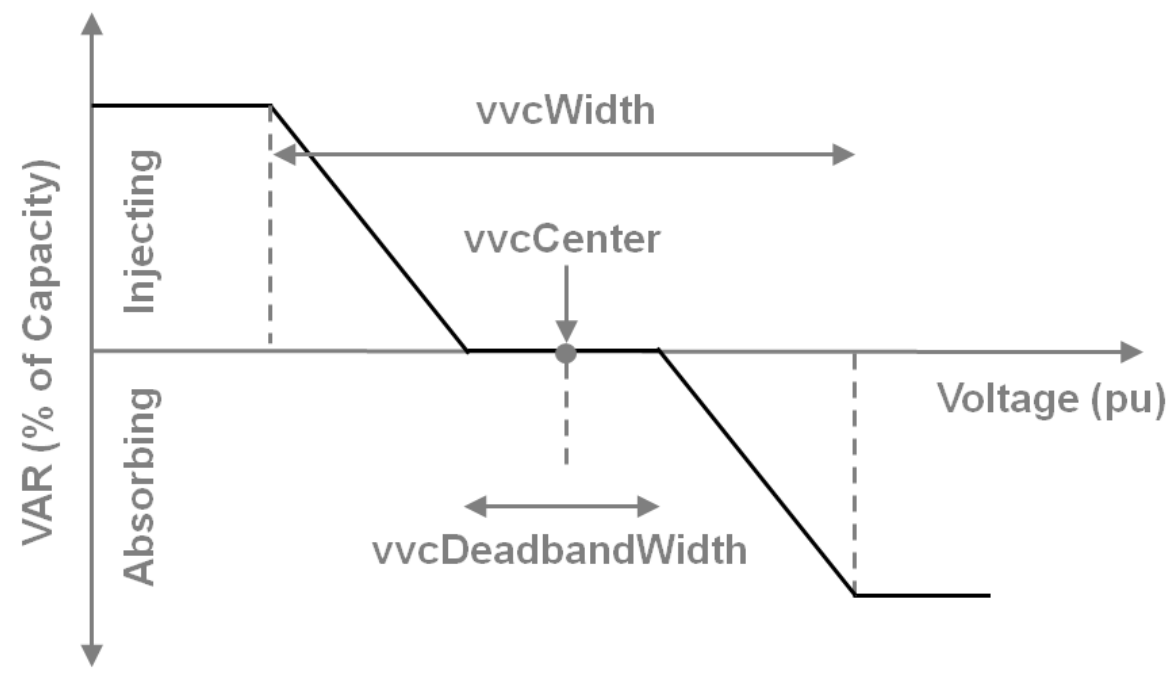

Figure 1. VVC setting illustration.

(In this study, the reactive power capability was limited to $60 \%$ of kVA nameplate.)

By setting the VVC center to a value less than 1 pu the curve will shift to the left. Previous studies have shown that feeder consumption savings can be realized with a lower curve center voltage, as the smart inverter pushes voltages toward the curve center [10].

The aim of this study was to determine the ability of distributed PV with smart inverters to provide additional voltage reduction energy savings. To measure the impact that smart inverters can have on voltage reduction schemes, this project examined if additional voltage reduction savings could be realized by adding randomly-located distributed PV with and without smart inverters. This was done at various PV penetration levels and various smart inverter densities within each penetration level. PV penetration is defined as the ratio of total PV power and peak loads in the system. For example, at a PV penetration level of $25 \%$ and smart inverter density of $50 \%$, there would be a $12.5 \%$ PV penetration with traditional inverters and a $12.5 \% \mathrm{PV}$ penetration with smart inverters. Simulations were run on the circuit models in the EPRI opensource load flow program called Open Distribution System Simulator (OpenDSS) [11] for one year at one hour intervals. Within OpenDSS, the programmed voltage reduction scheme chose the optimal LTC and capacitor positions to lower the voltage as much as possible. Details of this methodology follow in the next section of this report.

\subsection{Simulated CVR Voltage Optimization (VO) Algorithm}

A Python-based control algorithm was developed to implement a voltage optimized CVR scheme on the OpenDSS distribution system models. The python interfaced with the OpenDSS distributions model via the Windows Component Object Interface (COM). During the annual simulation, the Python code implemented the VO control scheme by adjusting the LTC and capacitors to achieve maximum energy savings. This was accomplished by finding the flattest and lowest voltage profile possible. Once, the optimum LTC tap position and capacitor bank switch positions were determined the Python simulation control code collected the simulation outputs, such as voltages and load demands. This control algorithm code was open-sourced along with this publication, to allow readers to apply the methodology to their own distribution system models. 
The VO methodology aimed to lower the voltage as much as possible with the LTC and capacitors. Voltage optimization (VO) schemes typically also optimize for reactive power which has the effect of minimizing losses and flattening the voltage profile potentially allowing greater aggregate voltage reduction on a feeder. This study focused on optimizing for the lowest voltage profile to realize maximum voltage reduction energy efficiency benefits at each customer's point of service. Future studies should look into optimizing for both energy savings at the points of service and the reactive power demand on the distribution system. This study's VO methodology was done in two steps:

1. Optimizing capacitors. Capacitor states were optimized to achieve the flattest voltage profile on the distribution system. The flattest voltage profile was defined as the voltage profile with the smallest difference between the maximum and minimum voltages. See below for a detailed diagram of the capacitor optimization algorithm.

2. Optimizing substation LTC. LTC tap position was optimized by selecting the lowest possible position without causing a voltage violation of less than $0.95 \mathrm{pu}$. As the capacitors flattened the profile in the previous step, the LTC was able to tap the voltage profile down to the lowest possible position. See Figure 2 for a flowchart of the algorithm.

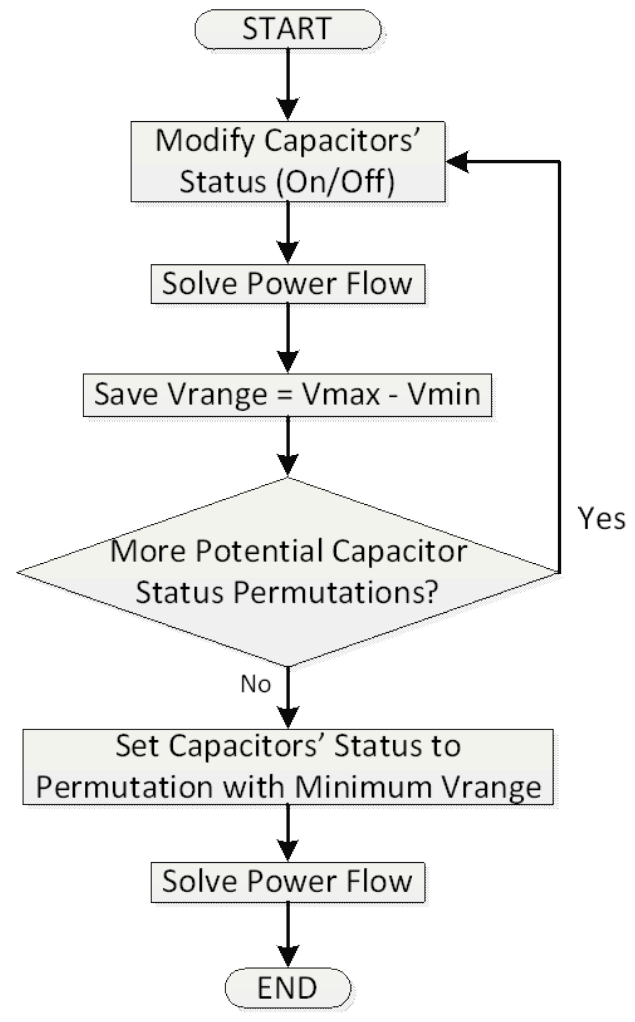

(a) Capacitor bank CVR optimization

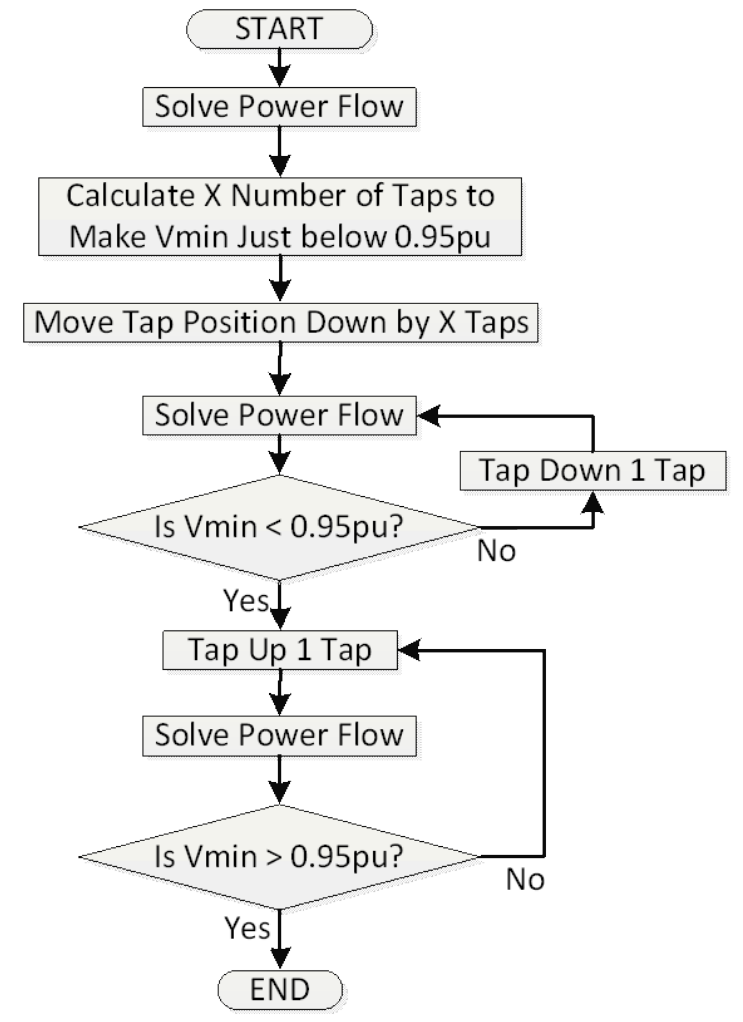

(b) Substation LTC CVR optimization

Figure 2. Optimization algorithms for capacitors and substation LTC 


\subsubsection{Smart Inverter Participation}

In addition to the LTC and capacitors, when smart inverters were included on a distribution system they provided one more way to tune the voltage profile. As previously mentioned, this study utilized the autonomous volt-VAR control available on the smart inverters to influence the distribution system voltage.

\subsubsection{Reactive Power Output Method}

When the volt-VAR control mode was enabled on a smart inverter there are two methods to produce reactive power: Watt priority and VAR priority. Under VAR priority mode, the production of reactive power was prioritized over the production of real power. This means that real power would be curtailed if there was not sufficient headroom in the inverter. For the purposes of this study, VAR-priority mode was selected to ensure that the inverters could influence the distribution system's voltage profile, even during peak solar output. Previous studies have oversized the inverters to ensure sufficient headroom for reactive power production. However, this does not necessarily reflect how inverter sizing is performed. Further discussion on inverter sizing is available in Section 4.3.2.

VAR-priority control was implemented using the Python control code because this functionality was not working as expected in OpenDSS at the time of the study. As shown in Figure 4 below, first OpenDSS solves for the inverter output using the software's built-in, properly functioning solver for Watt-priority. If reactive power was being curtailed, the Python code adjusted the real power output to allow for the full reactive power production. In this implementation, the active power curtailment was limited to $10 \%$ of the kVA rating of the inverter. A higher threshold could be used in future studies which would increase the amount of CVR energy saving and potentially increase the levels of PV curtailment amount, which was at maximum $1.3 \%$ of the annual PV output in this study (Section 6.3).

A maximum reactive power limit of $60 \%$, for a full-load power factor of 0.8 , was selected in order to represent the capability of the smart inverters currently available. When the smart inverter VVC called for reactive power that would exceed the apparent power limit of the inverter, the active power was curtailed by $10 \%$ to enable the majority of cases. Due to the curtailment simplification in the reactive power priority's implementation, active power was more likely to be over-curtailed than reactive power curtailed (i.e. the inverter was more likely to be below its kVA rating than at its kVAR rating). The smart inverter reactive power output determination is shown in Figure 3. Smart inverters were modeled to have reactive power capability at night, a capability that will soon be available. 


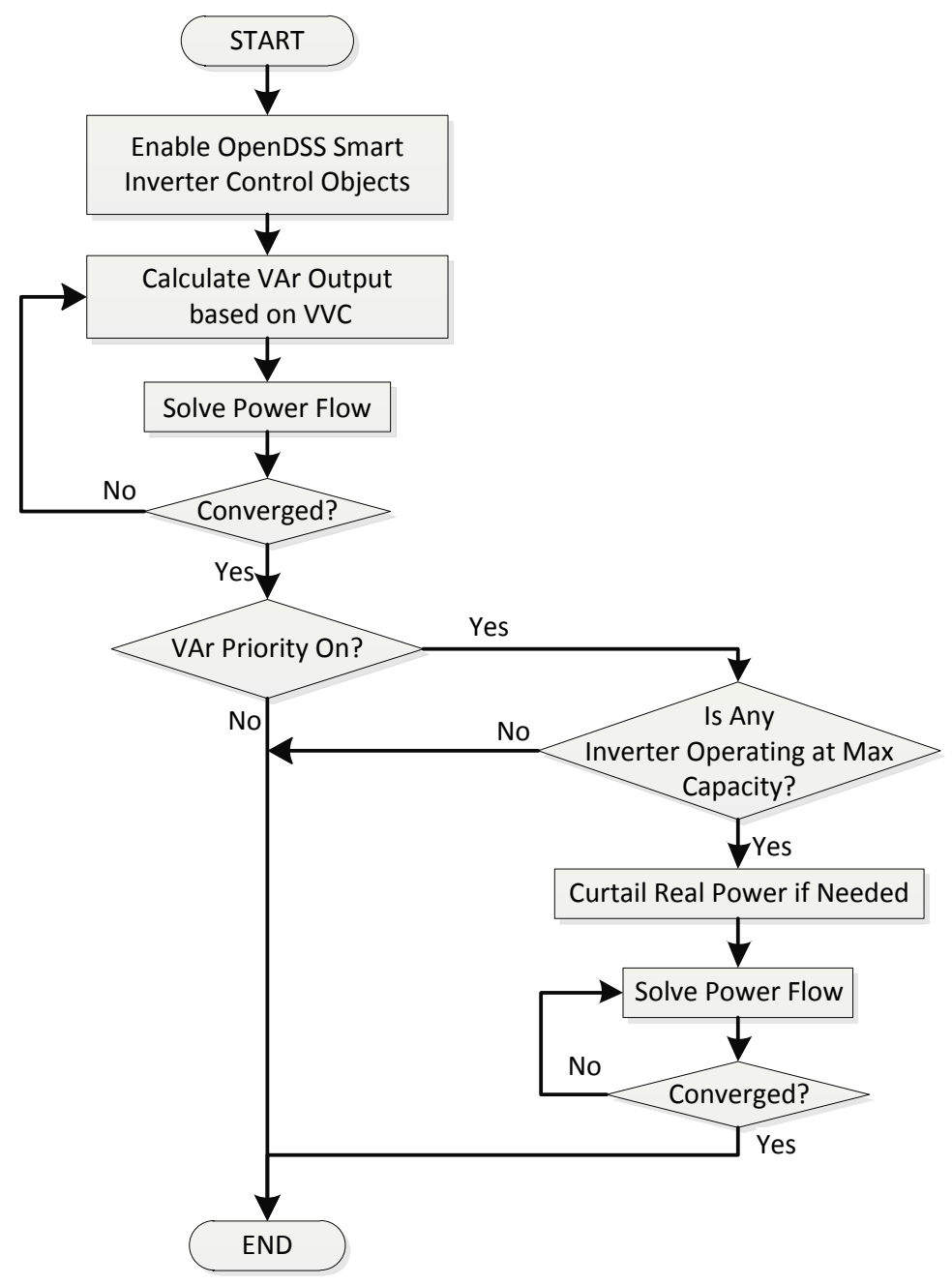

Figure 3. Smart inverter volt-VAR control output algorithm

\subsubsection{Smart Inverter Interaction with the CVR VO Scheme}

By allowing smart inverters to influence the voltage on a distribution system with a VO scheme, the intricacy of control scheme increased as the smart inverters, load tap changer, and capacitors could influence each other's states. By outputting reactive power and regulating their local voltage, smart inverters could cause the LTC to tap or the capacitor banks to switch. By tapping the LTC or changing a capacitor state, the voltage at the smart inverter would change, thus causing it to change its reactive power output. Modeling the interaction between these three voltage control devices in OpenDSS was quite complex as many possible methods were possible to converge on a solution. A number of design iterations were repeated before reaching the final implementation reported in this study. The final implementation of the VO scheme with smart inverters is shown in Figure 4. This was referred to as "local SI control" throughout the report. The sequence of the local control was:

1. Establish an initial solution without smart inverters. Execute the VO scheme by finding the capacitor and regulator state with the lowest voltage, under the condition that smart inverter volt-VAR support was disabled. 
2. Establish an initial solution with smart inverters. Enable the smart inverter's voltVAR control and adjust their reactive power output based on their VVC and local voltage. To ensure that this was a valid state, the algorithm checked that the distribution system voltages were all above 0.95 and below $1.05 \mathrm{pu}$. If a voltage violation occurred, the LTC position was tapped up or down by one position. The smart inverters then changed their output based on their new local voltage. If voltage violations still existed, this process was repeated. The first acceptable solution without violation would be the initial solution for the "local SI control."

3. Attempt to lower the voltage profile. Tap the LTC down and optimize the capacitors to determine if the voltage profile could be further flattened. The smart inverters then autonomously adjusted their output based on their VVC and local voltage. If this lowered profile was better than the initial solution with smart inverters, it was saved as the best solution. A better solution was defined as one having (1) a lower tap position, (2) a voltage profile without voltage violations, and (3) a lower system average voltage than the previous position. If a better solution was found, this step was repeated to see if the voltage profile could be lowered once more. If a better solution was not found, then the simulation advanced to the next time step.

A flowchart of this algorithm implemented in Python and OpenDSS is shown in Figure 4.

For the purposes of this paper, the energy consumption and other power quality metrics will be compared against a base case. This base case has $0 \% \mathrm{PV}$ penetration and therefore Smart Inverters off, and is simulated with CVR VO off (i.e. simulated with the existing settings of the voltage regulation equipment.) More details on the base case will be discussed in Section 2.3.

The energy savings and power quality of two scenarios will be compared in this paper: distribution system with controlled VO scheme and then that same VO scheme with smart inverters participating autonomously. This information is contained in Table 1 below.

Table 1. Simulated Scenarios with Different Control Settings

\begin{tabular}{llll}
\hline Studied Scenarios & PV Penetration & $\begin{array}{l}\text { Voltage Optimization } \\
\text { (Controlled LTC }+ \\
\text { Capacitor Banks) }\end{array}$ & $\begin{array}{l}\text { Smart Inverter } \\
\text { Participation }\end{array}$ \\
\hline Base Case & $0 \%$ & Off & Off \\
\hline $\begin{array}{l}\text { Voltage Optimization } \\
\text { Only }\end{array}$ & $0 \%-150 \%$ & On & Off \\
\hline $\begin{array}{l}\text { Local Control with } \\
\text { Smart Inverters }\end{array}$ & $0 \%-150 \%$ & On & On \\
\hline
\end{tabular}




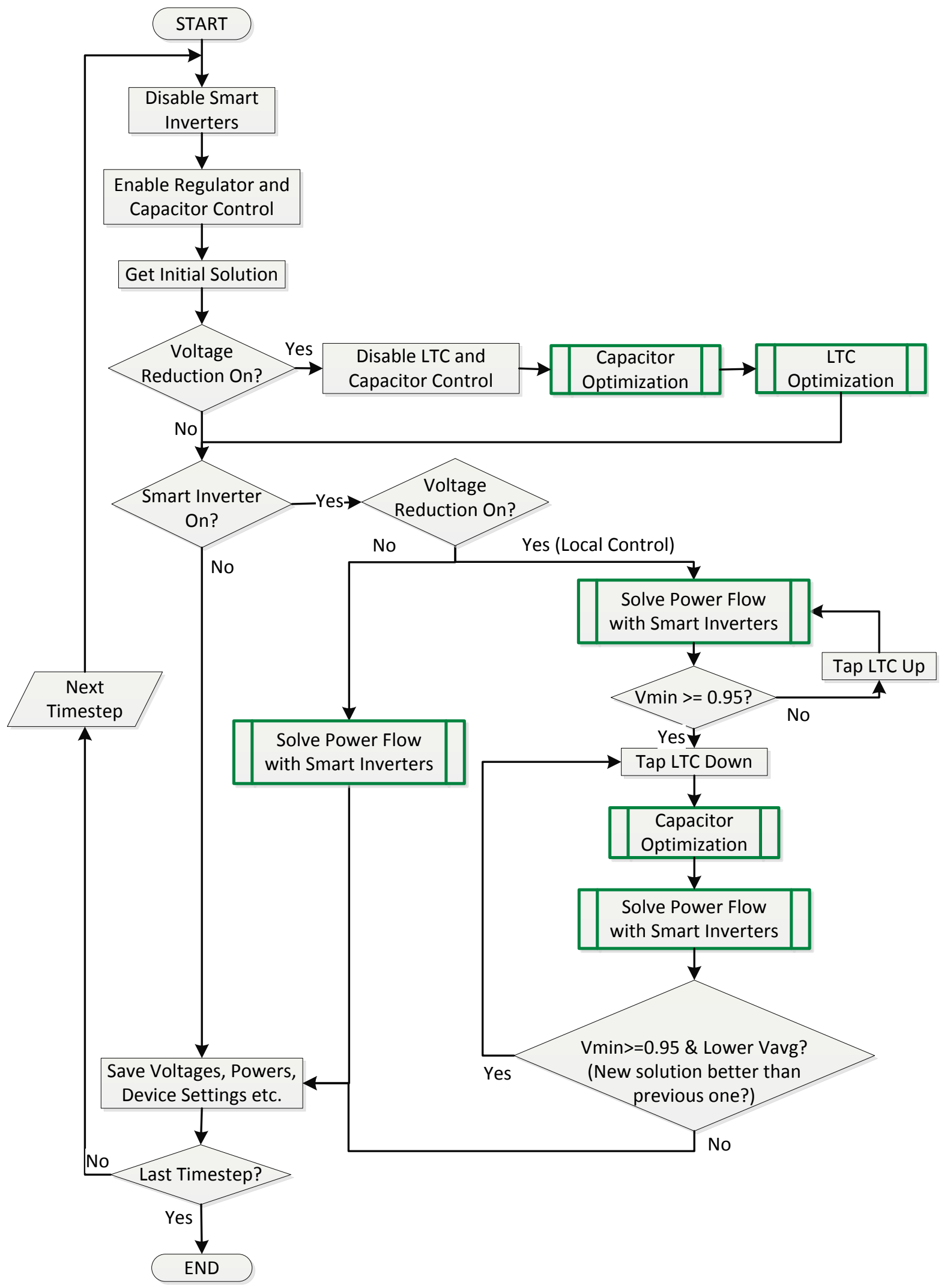

Figure 4. Local SI control: CVR VO algorithm with smart inverters 


\subsubsection{Demonstration of CVR Voltage Optimization Methodology}

Voltage snapshots are shown in Figure 5 for a winter day and Figure 6 for a summer day to demonstrate how the various functions of the VO algorithm work. In this scenario, the PV penetration was $5 \%$ and the smart inverter density was $25 \%$. As explained previously, this means there was a PV penetration with traditional inverters of $3.75 \%$ and a PV penetration with smart inverters of $1.25 \%$. VO steps are shown in figures (a) through (c). Figure (a) shows the starting voltage profiles without VO enabled. Figure (b) shows the profiles the flattened voltage profile after the capacitor optimization. Figure (c) shows the profile after the LTC optimization with the distribution system voltage as low as possible. The VO with local SI control continued from this state and is shown in Figure (d). It can be seen that even at this low PV penetration and smart inverters density ( $5 \% \mathrm{PV}$ and $25 \% \mathrm{SI})$, distributed $\mathrm{PV}$ with smart inverters were able to create enough of an impact to lower the LTC an extra step. These extra tap decrements were the main source for an increased voltage reduction energy savings as the distribution system operated at a lower voltage level. The voltage on the source side of the LTC was the source voltage to the entire distribution system and was the only voltage unchanged when the LTC tap position was modified. This was the single, higher node voltage seen in some of the figures.

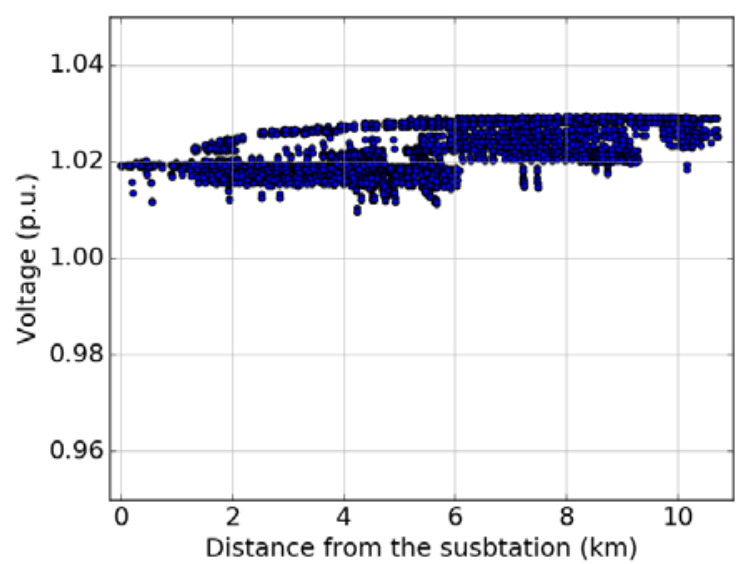

(a) Base case without CVR VO

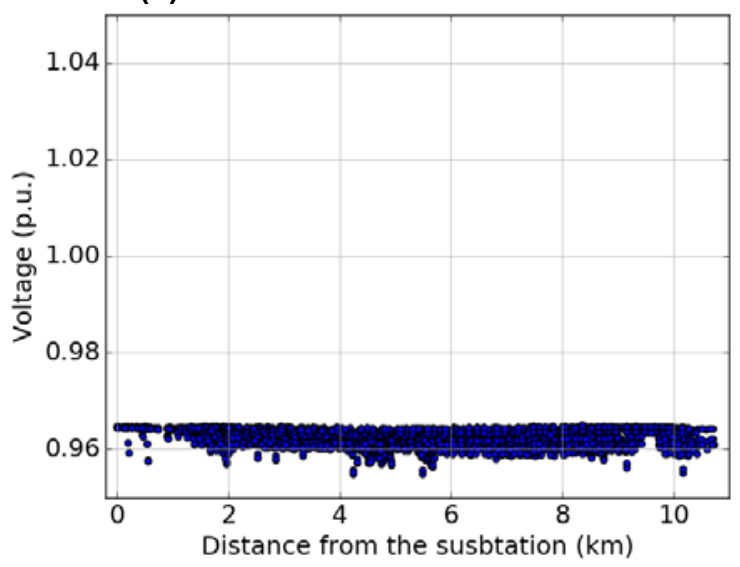

(c) CVR VO after LTC optimization

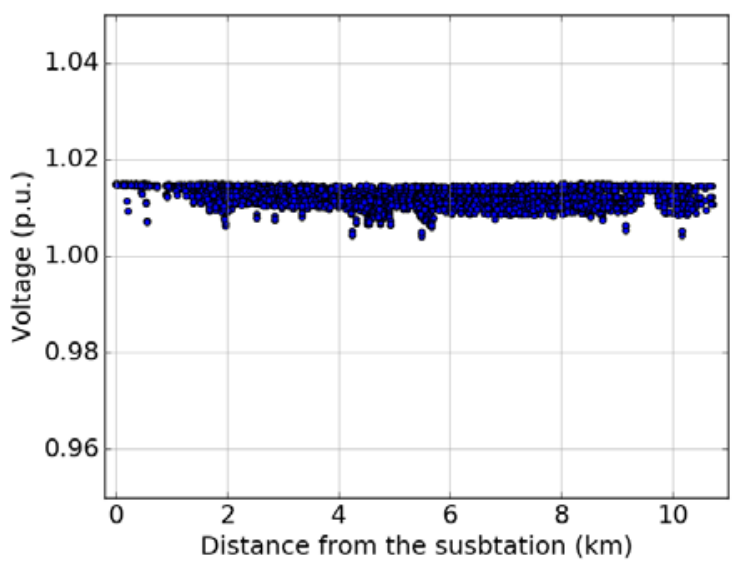

(b) CVR VO after capacitor optimization

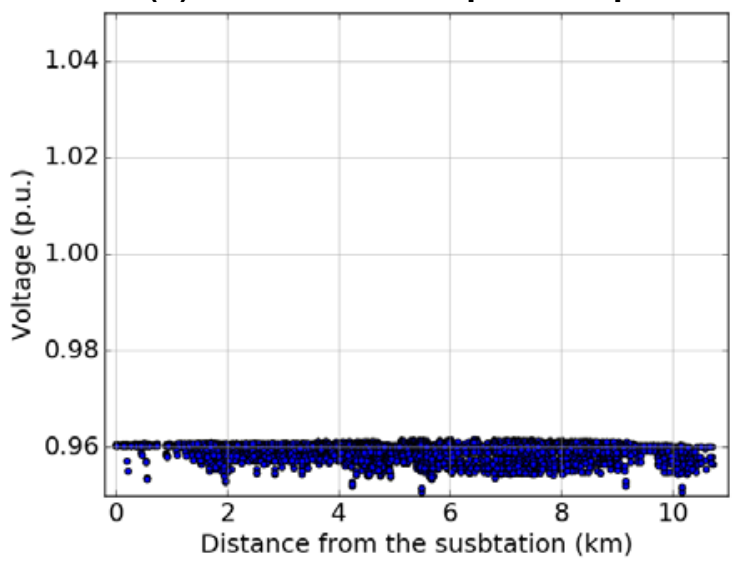

(d) CVR VO after local SI control

Figure 5. Voltage profiles for the PG\&E distribution system on a winter day demonstrating the various steps of the CVR VO algorithm 


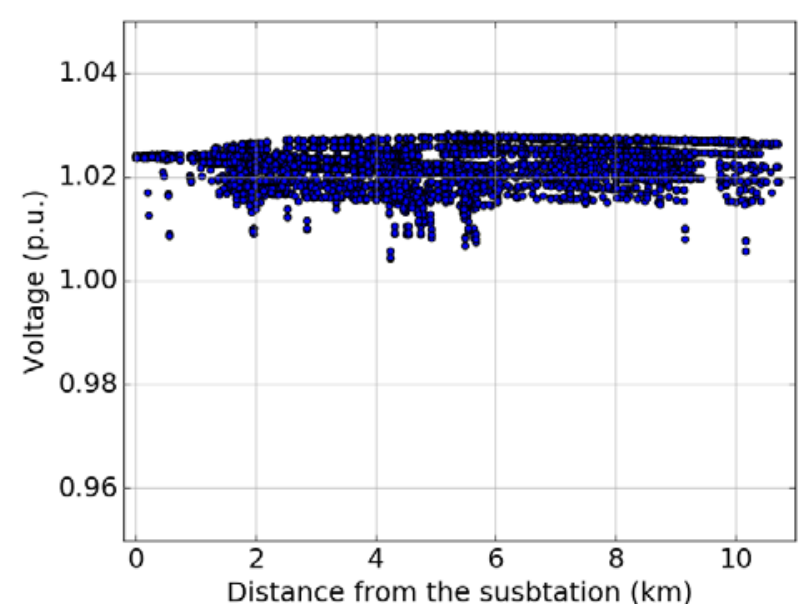

(a) Base case without CVR VO

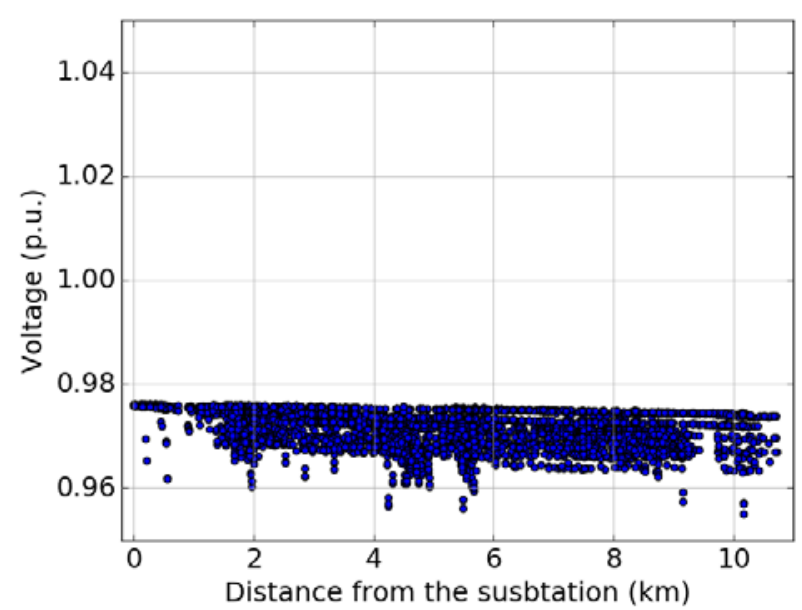

(c) CVR VO after LTC optimization

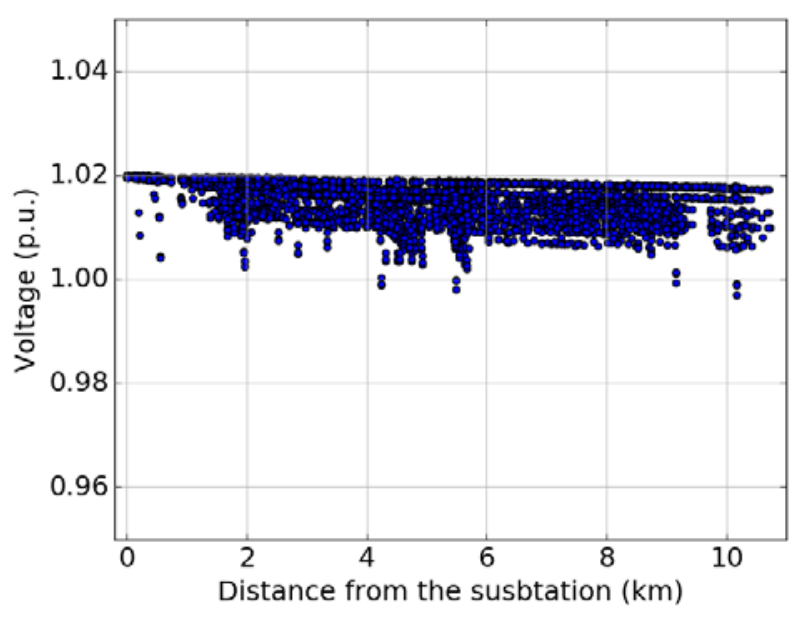

(b) CVR VO after capacitor optimization

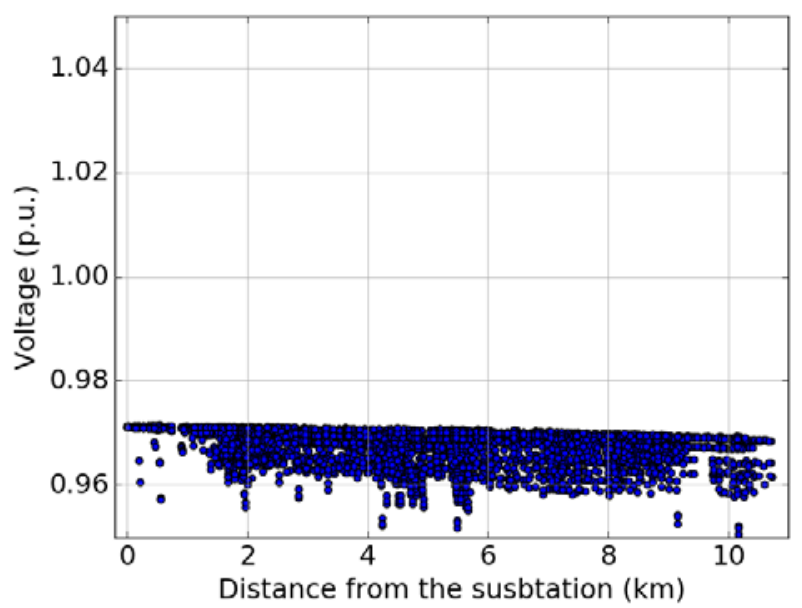

(d) CVR VO after local SI control

Figure 6. Voltage profiles for the PG\&E distribution system on a summer day demonstrating the various steps of the CVR VO algorithm

The voltage reduction energy savings from smart inverters for the whole year with 8,760 hourly steps are analyzed in detail in Section 5.

\subsection{Conservation Voltage Reduction Factor}

In the simulations, each load's energy consumption response to voltage changes was determined by the load's CVR factor. CVR factor is a metric used to describe the change in energy consumption as a function of a change in voltage. Specifically, CVR factor is the percent of change in energy consumption for a $1 \%$ reduction in distribution system voltage. When the voltage is lowered and the energy consumption is also lowered, this yields a positive CVR factor. A CVR factor value exists for both real and reactive power, though real power CVR factor is the most studied as it usually has the greatest economic implications for a utility and its customers. CVR factor for a specific distribution system is determined by its makeup of load. For real power, certain loads will reduce the amount of energy consume when the voltage is lowered, like 
heaters and light bulbs. Other loads can maintain their power consumption or in the case of plasma or liquid crystal display televisions, some loads can actually consume more energy when the voltage is lowered. The energy response of all the loads on the distribution system caused by a change in voltage determines its CVR factor.

Many studies have been completed on distribution efficiency and CVR factor. EPRI's report "Green Circuit: Distribution Efficiency Case Studies" [8] and Northwest Energy Efficiency Alliance's (NEEA) "Distribution Efficiency Initiative" [20] are commonly cited reports containing CVR factor research. In both of these reports distribution circuits from many different utilities were studied. The voltage on the feeder was lowered and the resulting energy response was recorded. In both of these studies real power CVR factor typically ranged from 0.5-0.9 and reactive power CVR factor typically ranged from 3-5. This is also supported by National Rural Electric Cooperative Association's (NRECA) "Costs and Benefits of Conservation Voltage Reduction" [22] which states that "decades of field research have found that for each 1\% reduction in distribution service voltage, mean energy consumption for residential and commercial customers is reduced by $0.8 \%$ ". Therefore for the purposes of this study, real CVR factor and reactive CVR factor were set to 0.8 and 4.0, respectively, for both utility models. In OpenDSS, these factors were implemented using two parameters: CVRwatts and CVRvars, which were the "percent reduction in active power (watts) and reactive power (VARs) respectively per $1 \%$ reduction in voltage from $100 \%$ rated." This study focused on real power CVR and therefore it should be assumed that CVR factor and CVR energy savings refer to the real power component only unless otherwise stated.

\subsection{Quantifying Voltage Reduction Energy Savings}

Voltage reduction energy savings were calculated from the reduction in load consumption at each customer. As PV impacts the energy consumption measured on the distribution system at the substation, the load demand from each customer was recorded at each simulation time step. Load demands for each customer were aggregated into a representative load demand for the distribution system. To determine the energy saved from voltage reduction, the load demand from each scenario was subtracted from the load from the base case without voltage reduction for each model. For both the HECO and PG\&E models, the base case had 0\% PV penetration with no smart inverters. Voltage reduction energy savings were calculated in terms of a value in MWh or as a percentage. The energy savings in MWhs was calculated as shown in Equation (1). For voltage reduction energy saving percentage, Equation (2) was used.

$$
\begin{gathered}
\text { EnergySaving }=\text { BaseLoad }_{0 \% P V, 0 \% S I, N o C V R}-\text { NewLoad } \\
\% \text { EnergySaving }=\frac{\text { EnergySaving }_{\text {BaseLoad }_{0 \% P V, 0 \% S I, N o C V R}}}{100 \%}
\end{gathered}
$$

where, BaseLoad ${ }_{0 \% P V, 0 \% S I, N o C V R}$ was the energy consumption obtained for the base case when there were no PV, smart inverter or voltage reduction. NewLoad was the new energy consumption obtained for other scenarios after voltage reduction energy savings. New Load is the gross load and is not inclusive of PV production. 


\section{Power Quality Scoring Methodology}

While there are many different components that make up power quality, a common methodology to incorporate these components into a single power quality score (PQS) did not exist. In addition to quantifying voltage reduction energy savings, this report documented a power quality scoring methodology that output a single score for the power quality "health" of a distribution system. Six different metrics were developed based on components that either directly impacted power quality or were often referenced in the context of power quality. The six indices that were used to compute the PQS were steady-state voltage, voltage fluctuation, voltage unbalance, control device operation, reactive power demand, and energy losses. In this version of the methodology, equal weight was given to each index. The proposed power quality scoring methodology could help assess the distribution system power quality and the impact of distributed PV with smart inverters on the system's power quality.

\subsection{Industry Standards for Power Quality}

Where possible, industry standards were used as the foundation of the power quality indices, including steady-state voltage, voltage unbalance, voltage fluctuation, and reactive power demand. In other areas, including energy loss and voltage control device operation, utility experience and available references were used. The sections below briefly describe the standards that were used for components that directly impact power quality.

\subsubsection{Steady-State Voltage}

The most commonly applied steady-state voltage standard in the United States is ANSI C84.12011 [6]. Within the standard, steady-state service voltage requirements are defined in two categories:

1. Range $\mathrm{A}$ is for normal conditions and the required regulation is $\pm 5 \%$ of nominal voltage ( 0.95 to 1.05 on a per unit base)

2. Range $B$ is for abnormal conditions and is intended to be for events that are limited in quantity and duration. The allowable range for these conditions is $-8.3 \%$ to $+5.8 \%$.

Both of these ranges were utilized in developing the Voltage Magnitude Violation Index as described in Section 3.2.1.

\subsubsection{Voltage Fluctuation and Flicker}

Voltage fluctuations sometimes give rise to noticeable illumination changes from lighting equipment. The regularity of these voltage fluctuations is much less than the 50 or $60 \mathrm{~Hz}$ supply frequency; however, they may occur with enough prevalence and magnitude to cause irritation for people observing the illumination changes. This phenomenon is referred to as flicker. Flicker is the subjective impression of fluctuating luminance caused by voltage fluctuations. Above a certain threshold, flicker becomes an irritant which can grow rapidly with the amplitude of the fluctuation. At a certain frequency of occurrence even very small amplitudes can be irritating.

Flicker severity or intensity is defined by the International Electrotechnical Commission (IEC) flickermeter, which aims to quantify an otherwise subjective phenomenon that varies from person to person. IEEE Recommended Practice 1453-2015 [12] adopted this standard, replacing the GE flicker curve in IEEE 519 and IEEE 141. It was determined that the GE flicker curve was 
not appropriate for measuring flicker from sources such as PV, as "such curves are suitable for step changes in RMS voltages, but are not suitable for predicting flicker caused by other sources...which are random in nature and have irregular wave shapes"[12].

Flicker measurements can be performed in real-time with a physical flickermeter installed at a measurement point or proactively simulated using a distribution model and high-resolution load and PV data. The PQS methodology aims to create a repeatable measurement process for annual simulations; however, the benefits of implementing the flickermeter may not outweigh the data collection and processing time necessary for its implementation. As a result, the methodology is instead focused on voltage fluctuation as described below. In the context of PV, studies have indicated that PV is not a significant source of flicker on the distribution system due to the frequency, magnitude, and ramp rate of PV power fluctuations [13],[14]. However, if there is a desire to evaluate PV's impact on flicker, screens can be performed using voltage deviation and/or the superseded flicker curve. When those screens fail, the flicker evaluation can continue on to more detailed simulations that rely on current standards and the flickermeter.

Voltage fluctuation is the difference in voltage magnitudes between time steps and is a simple concept that can be easily applied to the PQS. Unlike voltage deviation, voltage fluctuation does not add inherent bias toward a scenario without PV and, unlike the GE flicker curve, does not rely on an outdated methodology. As stated in IEEE 1250, "specific recommendations for voltage fluctuations are not provided in IEEE standards, but individual utilities usually have their own guidelines in the range of $4 \%$ to $7 \%$ " [15]. The percentage of fluctuation used to create the Fluctuation Index was more conservative than these values as described in Section 3.2.2.

\subsubsection{Voltage Unbalance}

Voltage unbalance defines the voltage difference between phases on a circuit. The voltage unbalance of a three-phase node was expressed as a percentage value and calculated as:

$$
\text { voltage unbalance }=\frac{\max \text { deviation from average } V}{\text { average } V} \cdot 100 \%
$$

where max deviation from average was the maximum difference between each of the three phases and the average of all three phases, in terms of RMS voltage.

Voltage unbalance causes increased heating in motors and can result in unbalanced currents and non-characteristic harmonics for electronic equipment like adjustable speed drives. ANSI C84.12011 [6] recommends that electric supply systems be designed and operated to limit the maximum voltage unbalance to $3 \%$ when measured at the electric-utility revenue meter under no load condition. This 3\% limit was used in developing the Voltage Unbalance Index described in Section 3.2.3.

\subsubsection{Reactive Power Demand}

Distribution system power factors that are outside of a standard operating range can have a negative effect on power quality, distribution system equipment, and can cause higher currents than would otherwise be necessary. Because PV systems with standard inverters output mostly active power, it can cause a decrease in the system power factor. PV systems with smart inverters are able to provide both real and reactive power, thus have the ability to provide reactive power 
support. In the PQS methodology, a reactive power demand was used instead of power factor. Maximum reactive power demand was based on IEEE Std. C57.12.00-2015 [17] and a power factor of 0.8 at rated transformer output ( $60 \%$ of the transformer rating). This standard was used to develop the Reactive Power Demand Index in Section 3.2.5.

\subsection{Power Quality Metrics}

The PQS was developed using the following system performance categories: steady-state voltage magnitude, voltage fluctuation, voltage unbalance, voltage control device operations, reactive power demand, and energy loss. These six categories were quantified into metrics that are described below:

- Metric 1: System Average Voltage Magnitude Violation Index (SAVMVI)

- Metric 2: System Average Voltage Fluctuation Index (SAVFI)

- Metric 3: System Average Voltage Unbalance Index (SAVUI)

- Metric 4: System Control Device Operation Index (SCDOI)

- Metric 5: System Reactive Power Demand Index (SRPDI)

- Metric 6: System Energy Loss Index (SELI).

Load demand and PV power output on distribution feeders are time-varying and thus quasi-static time-series (QSTS) analysis is necessary to measure the PQS. The sections following describe each metric in more detail.

\subsubsection{Metric 1: System Average Voltage Magnitude Violation Index}

Figure 7 shows an illustrative QSTS simulation result of steady-state voltage magnitude (RMS values) at a particular bus location, for one phase. All voltage magnitudes were normalized into per unit values. $V_{\min }(0.95 \mathrm{pu})$ and $V_{\max }(1.05 \mathrm{pu})$ were the minimum and maximum allowable values for voltage magnitude $\left(V_{\text {mag }}\right)$. The distribution system relies on an allowable range to regulate voltage and the PQS does not add inherent bias toward a particular voltage regulation practice, including voltage reduction schemes as may be implemented, so long as voltages are within the range defined by industry standards. All voltage magnitudes that lie in the allowable range, denoted as the shaded green area, satisfy power quality requirements and do not negatively impact SAVMVI. Voltage magnitudes that lie outside the allowable range violate standards and negatively impact SAVMVI.

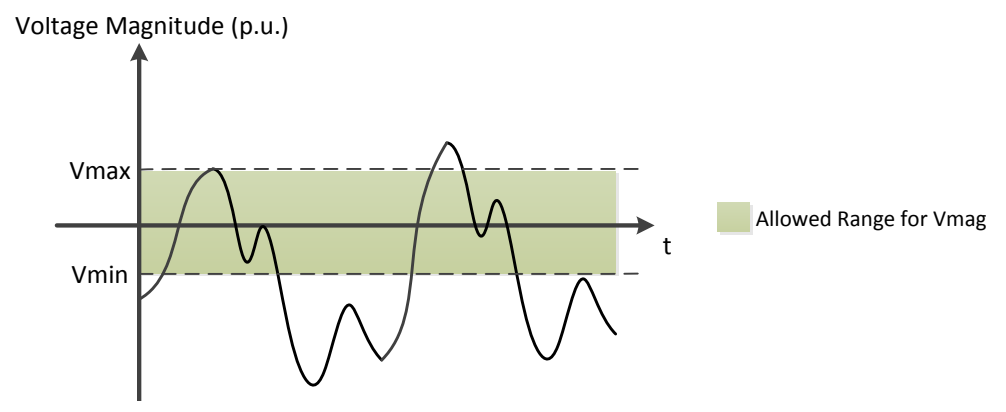

Figure 7. Illustrative QSTS result for steady-state voltage magnitude 
In order to capture the violating condition, the voltage magnitude violation, $V i o_{V_{m a g}}$, at one simulation time point was defined as:

$$
\operatorname{Vio}_{V_{\text {mag }}}(t)=\left\{\begin{array}{c}
V_{\text {max }}-V_{\text {mag }}, \text { if } V_{\text {mag }}>V_{\text {max }} \\
0, \quad \text { if } V_{\text {min }} \leq V_{\text {mag }} \leq V_{\text {max }} \\
V_{\text {mag }}-V_{\text {min }}, \text { if } V_{\text {mag }}<V_{\text {min }}
\end{array}\right.
$$

If a bus has two or three phases, the average value of all phase $\mathrm{Vio}_{V_{m a g}}$ was used as the voltage magnitude violation at this bus. All time steps were averaged for the entire time period $(T)$ and was given as $T V i o_{V_{\text {mag }}}$, the total $\mathrm{Vio}_{V_{\text {mag }}}$ in the equation below:

$$
T V i o_{V_{\text {mag }}}=\frac{\Sigma_{t=1}^{T}\left[\left(\Sigma_{\text {All phases }} \operatorname{Vio}_{V_{\text {mag }}}(t)\right) / \text { All Phases }\right]}{T}
$$

SAVMVI averaged the voltage magnitude violations for all $N$ buses.

$$
S A V M V I=\frac{1}{N} \cdot\left[\operatorname{TVio}_{V_{\text {mag }}}(\text { location } 1)+\cdots+\operatorname{TVio}_{V_{\text {mag }}}(\text { location } N)\right]
$$

The value obtained from Equation (6) was the raw value of the power quality metric SAVMVI. This raw value would be converted to a score for defining the final PQS.

\section{Minimum and Maximum Values of SAVMVI}

If SAVMVI was equal to zero, there were no voltage magnitude violations on the distribution system for the entire period evaluated. A higher SAVMVI means a poorer quality of voltage magnitude. ANSI Range-A for service voltage was used to define $V_{\min }$ and $V_{\max }$ in Equation (7). ANSI C84.1-2011 [6] specifies the maximum and minimum voltage for Range B as 127 $\mathrm{V}(1.0583 \mathrm{pu})$ and $110 \mathrm{~V}(0.9167 \mathrm{pu})$ respectively. While Range A was used to define what is considered a voltage violation, Range B was used to define the maximum possible violation as shown below.

$$
V I O_{V_{\operatorname{mag}}}^{\max }=\max \left\{1.0583-V_{\max }, V_{\min }-0.9167\right\}=0.0333 \text { p.u. }
$$

Therefore, the maximum value for SAVMVI was:

$$
\max (S A V M V I)=0.0333
$$

\subsubsection{Metric 2: System Average Voltage Fluctuation Index}

Voltage fluctuation was defined as the individual change in voltage magnitude (RMS value) before and after a time step. This is shown in Equation (10), where $V_{\text {mag }}^{i}(t)$ was the voltage magnitude in per unit of bus $i$ at time step $t$.

$$
\Delta V_{i}(t)=\left|V_{\text {mag }}^{i}(t+1)-V_{\text {mag }}^{i}(t)\right|
$$

SAVFI was defined as the average value of voltage fluctuations. 


$$
S A V F I=\frac{1}{N} \cdot\left(\frac{1}{T} \sum_{i=1}^{N} \sum_{t=1}^{T} \Delta V_{i}(t)\right)
$$

$\Delta V_{i}(t)$ was the voltage fluctuation at time step $t$ for the observed location $i$; $T$ was the entire simulation time points; $N$ was the total number of locations observed.

\section{Minimum and Maximum Values of SAVFI}

If SAVFI was equal to zero, each node voltage was constant for the entire time period. Otherwise, a higher SAVFI means a lower power quality with larger voltage fluctuations.

Voltage changes due to events such as motor starting, capacitor switching and voltage regulator switching are classified as rapid voltage changes (RVC) sustained over several cycles. IEEE 1453-2015 [12] listed the indicative planning levels for rapid voltage changes by inheriting from IEC Standard 61000-3-7. A 3\% limit is recommended for voltage fluctuations occurring 2 to 10 times per hour in medium-voltage systems. While this $\mathrm{RVC}$ recommendation does not relate specifically to fluctuation, this value was used as the maximum value of $\Delta V_{i}(t)$. Therefore, the maximum value of SAVFI was:

$$
\max (S A V F I)=0.03
$$

\subsubsection{Metric 3: System Average Voltage Unbalance Index}

SAVUI was defined as the average value of voltage unbalances for all three-phase buses. This is shown in Equation (12), where, $V_{i m b}^{i}(t)$ was the voltage unbalance at time step $t$ for the observed three-phase location $i$; $T$ was the entire simulation time points; $N$ was the total number of locations observed.

$$
S A V U I=\frac{1}{N} \cdot\left(\frac{1}{T} \sum_{i=1}^{N} \sum_{t=1}^{T} V_{i m b}^{i}(t)\right)
$$




\section{Minimum and Maximum Values of SAVUI}

If SAVUI was equal to zero, three-phase voltages throughout the feeder were perfectly balanced for the entire time period. A higher SAVUI means a lower power quality with increased voltage unbalance.

During normal operations, the three-phase voltages are within 0.95 to $1.05 \mathrm{pu}$ Therefore, the most unbalanced scenario would occur if the three-phase voltages are at the two opposite extremes. The unbalance level in this scenario is shown in Table 2 as $0.0678 \mathrm{pu}$ when two phase voltages were $0.95 \mathrm{pu}$ and the other phase voltage was $1.05 \mathrm{pu}$

Table 2. Voltage Unbalance at Extreme Scenario

\begin{tabular}{ccccc}
\hline \multicolumn{2}{c}{ Three-Phase Voltages $(\mathbf{p u})$} & Average & Voltage Unbalance \\
\hline 0.95 & 0.95 & 1.05 & 0.9833 & 0.0678 \\
0.95 & 1.05 & 1.05 & 1.0167 & 0.0656 \\
\hline
\end{tabular}

Therefore, the maximum value of SAVUI is:

$$
\max (S A V U I)=0.0678
$$

\subsubsection{Metric 4: System Control Device Operation Index}

The operation of voltage regulators and operation of capacitors were separated in order to appropriately characterize each type of voltage control device. $S C D O I_{V R}$ was defined as the Regulator Operation Index and $S C D O I_{C a p}$ was defined as Capacitor Operation Index.

\section{Regulator operation index}

Let $T O_{V R, i}$ be the total number of tap changes of the $i$-th voltage regulator for the entire studied time period. At each simulated time, a voltage regulator could have 0 tap change or multiple tap changes, with a typical maximum of 32 tap changes.

And the Regulator Operation Index, $S C D O I_{V R}$ was defined based on a daily scale, as shown in Equation (14), where, $N R$ was total number of voltage regulators and Tday was the total days studied.

$$
S C D O I_{V R}=\frac{\sum_{i=1}^{N R} T O_{V R, i}}{T d a y \cdot N R}
$$




\section{Minimum and Maximum Values of SCDOI $\mathrm{VR}$}

If $S C D O I_{V R}$ was equal to zero, it means no tap changes occured during the entire time period, which was considered as the best scenario for device operation. A larger non-zero value of $S C D O I_{V R}$ indicates more control device operations were needed to regulate voltage, potentially leading to increased mechanical wear on the device.

The extreme value of the total number of tap changes for one regulator at each time step is 32, but this rarely occurs. Electrical Power Transformer Engineering [15] provided the list of number of LTC switching operations in various fields of application shown in Table 2.

Table 3. Number of LTC Switching Operations in Various Fields of Application [16]

\begin{tabular}{ccccccc}
\hline \multirow{2}{*}{ Transformer } & \multicolumn{3}{c}{ Transformer Data } & \multicolumn{2}{c}{$\begin{array}{c}\text { Number of On-Load Tap Changer } \\
\text { Operation Per Year }\end{array}$} \\
\cline { 2 - 7 } & $\begin{array}{c}\text { Power } \\
(\mathrm{MVA})\end{array}$ & $\begin{array}{c}\text { Voltage } \\
(\mathrm{kV})\end{array}$ & Current (A) & Min. & Median & Max. \\
\hline Power Station & $100-1300$ & $110-765$ & $100-2000$ & 500 & 3000 & 10,000 \\
\hline Interconnected & $200-1500$ & $110-765$ & $300-3000$ & 300 & 5000 & 25,000 \\
\hline Network & $15-400$ & $60-525$ & $50-1600$ & 2000 & 7000 & 20,000 \\
\hline Electrolysis & $10-100$ & $20-110$ & $50-3000$ & 10,000 & 30,000 & 150,000 \\
\hline Chemistry & $1.5-80$ & $20-110$ & $50-1000$ & 1000 & 20,000 & 70,000 \\
\hline Arc Furnace & $2.5-150$ & $20-230$ & $50-1000$ & 20,000 & 50,000 & 300,000 \\
\hline
\end{tabular}

This report considered 10,000 as the maximum number of tap changes for one regulator per year. Therefore, the maximum value of $S C D O I_{V R}$ for a day was:

$$
\max \left(S C D O I_{V R}\right)=\frac{10000}{365} \approx 30
$$

\section{Capacitor operation index}

Let $T O_{C a p, j}$ be the total number of operations of the $j$-th switched capacitor bank for the entire studied time period. Similar to the Regulator Operations Index, the Capacitor Operation Index, $S C D O I_{C a p}$, was defined in Equation (16), where $N C$ was total number of switched capacitor banks in the feeder and Tday was the total days studied.

$$
S C D O I_{C a p}=\frac{\sum_{j=1}^{N C} T O_{C a p, j}}{\text { Tday } N C}
$$

\section{Minimum and Maximum Values of SCDOI Cap $_{\text {ap }}$}

If $S C D O I_{C a p}$ was equal to zero, it means no capacitor switching operations occurred during the entire time period, which was considered as the ideal scenario for device operation. A larger non-zero value of $S C D O I_{C a p}$ indicates more capacitor switching operations have occurred. The capacitor is not designed to operate as often as voltage regulators and typically each capacitor has a maximum number of operations per day, denoted as CapNday. Thus, the maximum value of $S C D O I_{C a p}$ was computed as shown below. In the case study following, a CapNday value of 4 was used.

$$
\max \left(S C D O I_{C a p}\right)=\text { CapNday }
$$




\subsubsection{Metric 5: System Reactive Power Demand Index}

The average of reactive power demands from the substation during the entire simulation time was used to define SRPDI. SRPDI is shown in Equation (18), where, $Q_{\text {sub }}^{t}$ was the substation reactive power demand at time point $\mathrm{t}$ and $T$ was the number of time points simulated.

$$
S R P D I=\frac{1}{T} \sum_{t=1}^{T} Q_{\text {sub }}^{t}
$$

\section{Minimum and Maximum Values of SRPDI}

If SRPDI was equal to zero, there was no reactive power demand from the substation at all times, with a substation power factor of exactly 1.0. As SRPDI increased, a lower power quality score was given. IEEE Std C57.12.00 ${ }^{\mathrm{TM}}-2015$ [17] states that a transformer's rating is based on a load power factor of $80 \%$ or higher. Therefore, $60 \%$ of substation transformer $\mathrm{kVA}$ rating was used as the maximum allowable reactive power demand at the substation. Therefore, the maximum value for SRPDI is shown in Equation (19), where $S_{\text {subxfmr }}$ was the MVA rating of the substation transformer

$$
\max (S R P D I)=0.6 \cdot S_{\text {subxfmr }}
$$

Note: if measuring PQS for a single circuit on a multi-circuit substation transformer, the denominator $\mathrm{S}_{\text {subxfmr }}$ was distributed based on the peak load. For instance, if an $X$ MVA substation transformer was serving circuit-A with $Y$ peak load and circuit-B with $Z$ peak load, the $\max (\mathrm{SPRDI})$ for circuit-A would be $0.6 \cdot X \cdot[Y /(Y+Z)]$.

\subsubsection{Metric 6: System Energy Loss Index}

System energy loss was included as another metric to evaluate power quality. Total energy loss during the entire simulation time was calculated and the total load demand was used as the dominator to normalize the energy loss. SELI was defined in Equation (20) where, $E_{\text {loss }}$ was total energy loss during the entire simulation time; $E_{\text {load }, i}$ was the energy demand of load- $i$ throughout entire simulation time; $L N$ was the total number of loads observed.

$$
S E L I=\frac{E_{\text {loss }}}{\sum_{i=1}^{L N} E_{\text {load }, i}}
$$

\section{Minimum and Maximum Values of SELI}

The minimum value of SELI was equal to zero, indicating an ideal system with no energy loss. A larger SELI value would lead to a lower power quality score. An approximate range from $1.5 \%$ to $9 \%$ was observed by EPRI Green Circuit projects [8], thus $9 \%$ was considered as the maximum value of SELI.

$$
\max (S E L I)=0.09
$$

\subsection{Power Quality Score}

PQS was determined based on the metric values of SAVMVI, SAVFI, SAVUI, SCDOI, SCDPI and SELI. The individual raw score for each power quality metric was determined based on the 
equations previously described in Section 3.2, before aggregating them up into a single power quality score.

\subsubsection{Step 1: Determine Individual Power Quality Metrics}

Figure 8 shows the linear correlation between the power quality metric value and the individual score. The minimum value of each metric indicated the best power quality and the score was given as 10 . The maximum value of each metric indicated the worst power quality and the score was given as 0 . Any other value of each metric was given an individual score calculated using the linear correlation as shown in Equation (22). If no minimum is listed for a particular metric in Section 3.2 above, then it was assumed to be zero. Following later in the report is a discussion about how the definition of the maximum and minimum value of each individual score can influence the overall PQS, potentially inadvertently giving more or less weight to each of the individual scores.

$$
\text { Individual Score }=10-\frac{\left.10 \cdot\left(\text { value } \text { Metric }_{i}\right)-\min \left(\text { Metric }_{i}\right)\right)}{\max \left(\text { Metric }_{i}\right)-\min \left(\text { Metric }_{i}\right)}
$$

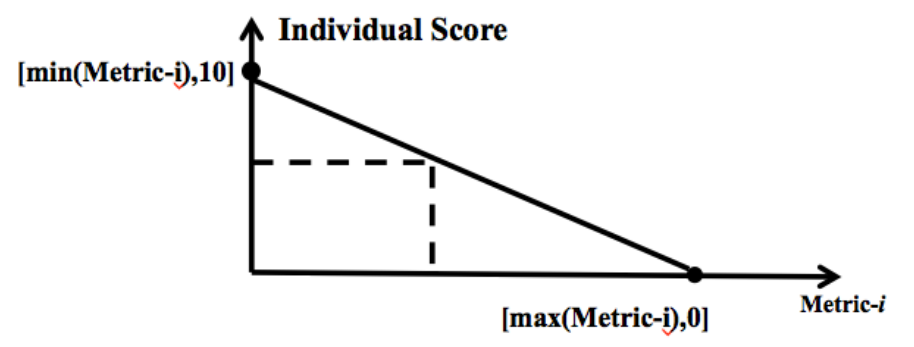

Figure 8. Linear correlation between power quality metric value and its individual score

\subsubsection{Step 2: Determine the Final PQS}

PQS was the linear combination of six individual scores, as shown in Equation (23). S(.) was the function used to represent the individual score, which was determined in Step 1 and $\alpha$ was the weight given to each index. The summation of all weights was equal to 1 . The case study that follows assumed that all six metrics were equally important. Therefore, a value of $1 / 6$ was given to each weight.

$$
\begin{aligned}
P Q S= & \alpha_{S A V M V I} \cdot S(S A V M V I)+\alpha_{S A V F I} \cdot S(S A V F I)+\alpha_{S A V U I} \cdot S(S A V U I)+ \\
& \alpha_{S C D O I} \cdot S(S C D O I)+\alpha_{S R P D I} \cdot S(S R P D I)+\alpha_{S E L I} \cdot S(S E L I)
\end{aligned}
$$




\section{Simulation Methodology}

The framework for simulation is presented in this section to prepare the model for the annual simulation. The steps included converting the utility models to OpenDSS, adding secondary circuits, modeling PV systems and smart inverters, and selecting the optimal VVC.

To simulate the voltage reduction scheme and evaluate feeder power quality, a Python simulation control code and OpenDSS solver were used to implement the CVR VO scheme. Python is an open-source high level programming language that is popular in research and academic communities. OpenDSS is a tool for performing distribution feeder modeling and power flow simulations. This simulator is an open source load flow program designed and maintained by EPRI. The tool has long been used by research entities and some utilities to model distribution feeders and perform analyses. As it is open source, it allows for results and methods to be evaluated and built upon by any interested party.

OpenDSS has many useful features that were employed by this study, including 8,760 -hour timeseries simulations and customizable advanced inverter functions, thus allowing for a quasi-static time series (QSTS) analysis to be completed.

\subsection{Model Conversion}

Synergi Electric [18] and CYMDIST [19] are two distribution system simulation software packages that are widely used by utilities. The distribution system models obtained from HECO and PG\&E were in Synergi and CYMDIST format respectively. NREL previously developed two model conversion software packages used as a part of this study that automatically convert a feeder model in Synergi or CYMDIST formats to OpenDSS [20]. These two model conversion tools are Synergi2DSS and CYME2DSS. Figure 9 describes the steps of the model conversion tools. These tools were coded using Python, which read the model files generated from Synergi and CYMDIST and then translated the information into OpenDSS.

In this project, new code was added to the existing model conversion software packages so that secondary circuit can be included in the feeder model automatically based on the load information. Additionally, the previous CYME2DSS was updated to be able to convert the models included in the most recent version of CYMDIST, version 7.2. After the conversion to OpenDSS and the addition of the secondary lines, the model contained primary and secondary feeder elements from the low side of the substation transformer down to the customer loads on the secondary side of the distribution service transformer. 


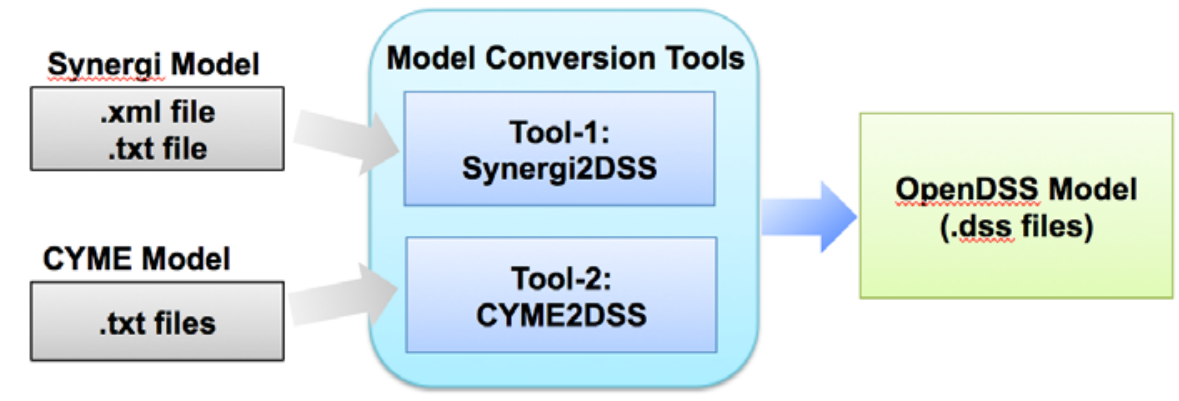

Figure 9. Framework of the model conversion Tool

\subsubsection{Model Conversion: Synergi and CYMDIST to OpenDSS}

The Synergi to OpenDSS conversion (Synergi2DSS) took network configuration data (.xml file) and line configuration data (.txt file) as inputs. Synergi2DSS read the XML feeder model file to identify, collect, and categorize all circuit "objects" and their properties. As shown in Figure 10, the objects were identified by the symbol " $<$ ", such as object "Node" and object "Feeder". The properties for objects were on indented lines below each of these, such as "NodeId", "X", "Y", "Description", "IsPadMountGear" included in the object "Node". Once the objects and properties were collected, Synergi2DSS wrote OpenDSS scripts to create the corresponding OpenDSS models. Similarly, Synergi2DSS read the text file containing the information of line configurations to define the line parameters in the converted OpenDSS model.

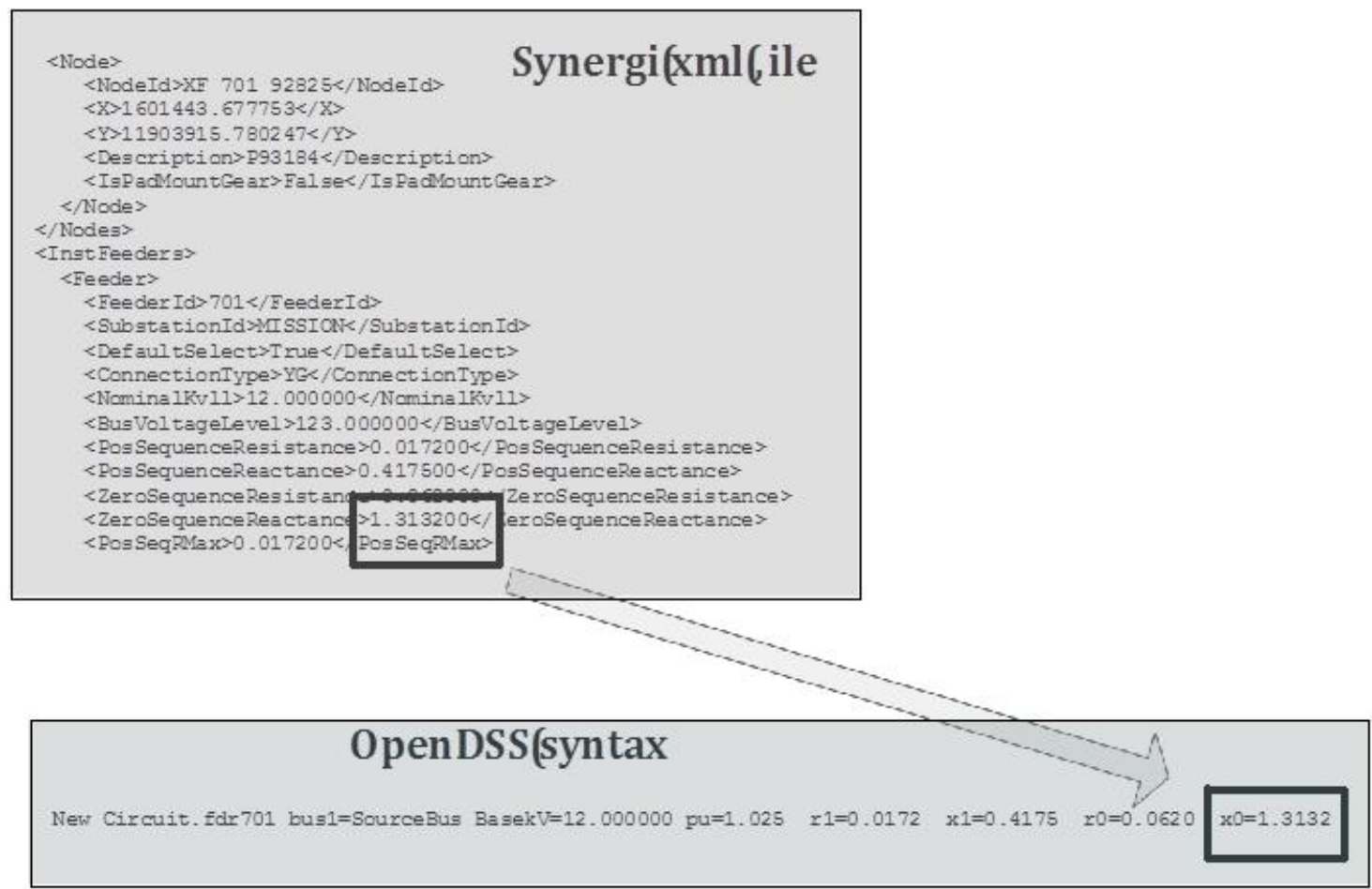

Figure 10. Diagrammatic view of Synergi to OpenDSS model conversion depicting the syntax identification process 
The process of converting objects in Synergi to the equivalent objects in the OpenDSS script was not always a direct one-to-one conversion. Object types and properties that existed in Synergi did not always exist in OpenDSS. For example, switches, reclosers, and fuses were not modelled as separate objects in OpenDSS, and instead were added as components to line objects. The conversion tool created short, low-impedance lines with appropriate capabilities to account for these components.

The converted OpenDSS model was written into a master circuit DSS file and separate DSS component files representing distribution system elements. The master circuit file initiated a new circuit with a source bus. The voltage and source impedances were specified based on data from the Synergi model. The master file also pointed to the DSS component files containing circuit details for the different object types.

The CYMDIST to OpenDSS conversion (CYME2DSS) had similar logic to the Synergi2DSS conversion. The CYMDIST distribution system model from PG\&E was first opened in CYMDIST and then exported into three ASCII text files including those for the equipment, loads, and network. All model information was contained in these text files, and the CYME2DSS conversion tool extracted the information and translated it into OpenDSS model files.

\subsection{Secondary Circuit Modeling}

After the models were converted to OpenDSS, secondary systems were added to each of the two utility distribution system models. In the original model from each utility, customers on a distribution transformer were aggregated into a single load connected on the primary, medium voltage distribution system. Customer counts and customer power factor assumptions were included in these models. Using this information, these primary spot loads were removed and replaced with representative secondary models. Each added secondary consisted of a distribution transformer, secondary conductor, and the various customer loads.

For the distribution transformers, impedances were provided by the utility and were assigned based on the transformer primary and secondary voltages and the apparent power rating of the transformer.

From the distribution service transformer, secondary lines were connected that extended out to the customer loads. Because there was limited information about a typical secondary for the PG\&E model, all customers were placed at the end of a single secondary conductor to study the worst case voltage drop across the secondary. The length of the secondary conductor used was 50 feet. For the HECO model, more information on the typical secondary configuration was available and each residential and commercial customer received their own secondary conductor. The length of the secondary conductor was either 60 or 100 feet.

The code function of adding the secondary circuits into the distribution system model first read the XML file (for Synergi) or text file (for CYMDIST) to identify the necessary information, including the number of customers on the secondary, the distribution transformer size in kVA and the customer load sizes in $\mathrm{kW}$ and $\mathrm{kVAR}$. Based on the collected information, the secondary circuit was model as: 
- Total number of loads connected at each distribution transformer secondary node was determined by the number of customers obtained from XML or text file. Each customer's load demand was computed as the total value of load $\mathrm{kW}$ and $\mathrm{kVAR}$ collected from the XML or text file divided by the total number of customers.

- A single distribution transformer delivered power to all the customers downstream on the secondary. The size of the transformer was the value of distribution transformer kVA collected from the XML or text file.

- A secondary line was created to connect a secondary transformer and a secondary load. Line length and conductor configuration were based on conversations with each utility.

Figure 11 shows an example of identifying property information and developing secondary circuit model for Synergi feeder model. The developed secondary circuit model was written into .DSS files as the converted OpenDSS model.

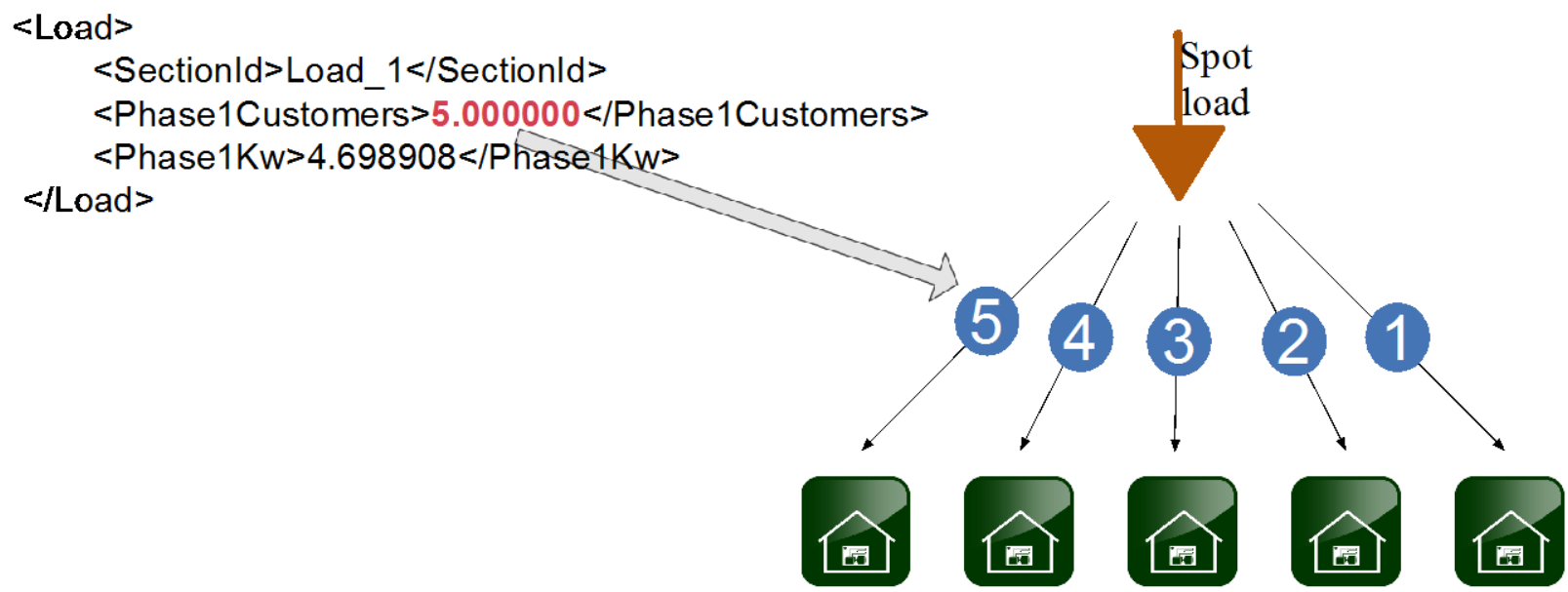

Figure 11. Diagrammatic view of Synergi to OpenDSS secondary addition depicting the syntax identification process

\subsection{Photovoltaic System Modeling}

For this study, preexisting PV systems were removed from the original models. A new PV system was added to every load, which were used to model various PV penetration levels on the feeders. The PV systems were modeled using the OpenDSS PVSystem object and smart inverters were implemented using the OpenDSS InvControl object. The size and allocation of the added PV systems and smart inverters were determined using the methodologies detailed in the following section. Once the PV was sized and allocated, a smart inverter was added to each PV system. Different PV and smart inverter penetrations levels were achieved by turning on certain distributed PV and smart inverters based on a randomly allocated order. More detail is included in the following sections.

\subsubsection{Photovoltaic Size Determination: Annual Load Offset}

PV systems were sized based on an $80 \%$ offset of the annual load consumption. For example, if a load consumes $10,000 \mathrm{kWh}$ a year. An $80 \%$ load offset for PV means that over that simulated year, total PV generation output is $8,000 \mathrm{kWh}$. 
The load consumption was calculated by multiplying the annual load consumption per $\mathrm{kW}$ with the peak $\mathrm{kW}$ rating of the load. The $\mathrm{kW}$ size of the PV system was determined by dividing the load consumption by the annual PV production per kW PV rating given from the normalized PV profile (Equation 24).

$$
P V\left[k W_{D C}\right]=\frac{\text { Normalized Annual Load Consumption }\left[\frac{k W h}{k W}\right] * \text { Load } k W \text { Rating }[k W]}{\text { Normalized PV Annual Output }\left[\frac{k W h}{k W_{D C}}\right]}
$$

$P V\left[k W_{D C}\right]$ is the size of the PV system in $\mathrm{kW}_{\mathrm{DC}}$ to be added to the according load. Normalized Annual Load Consumption is the annual energy consumption (kWh) of the load profile provided for the load per kW of load. Normalized PV Annual Output is the annual energy output $(\mathrm{kWh})$ of the PV system based on the provided PV profile per $\mathrm{kW} \mathrm{DC}_{\mathrm{D}}$ of PV.

\subsubsection{Inverter Sizing}

The apparent power rating for each inverter was determined by the applying the ratio of the inverter (AC) and the PV panels (DC). PV systems produce energy at nameplate $\mathrm{kW}$ for a subset of daylight hours in a year and the cost of an inverter is not immaterial. Therefore, for economic reasons, solar developers often size inverters smaller than the PV panel nameplate $\mathrm{kW}$. The DC to $\mathrm{AC}$ ratio used in this study was 1.25 .

Reactive power capability of smart inverters followed current device ratings, which was limiting the amount of VAR to $0.6 \mathrm{pu}$ or $60 \%$ of the inverter capacity for volt-VAR control. That is to say, the minimum power factor limit was 0.8 when the inverter operated at its $\mathrm{kVA}$ rating. When PV output was less than $80 \%$ of the nameplate kVA rating, no curtailment was needed in VARpriority mode, as could be seen in Figure 12. As described in Section 2.1.1.1, reactive power was provided at night and active power was curtailed by $10 \%$ when additional reactive power was needed.

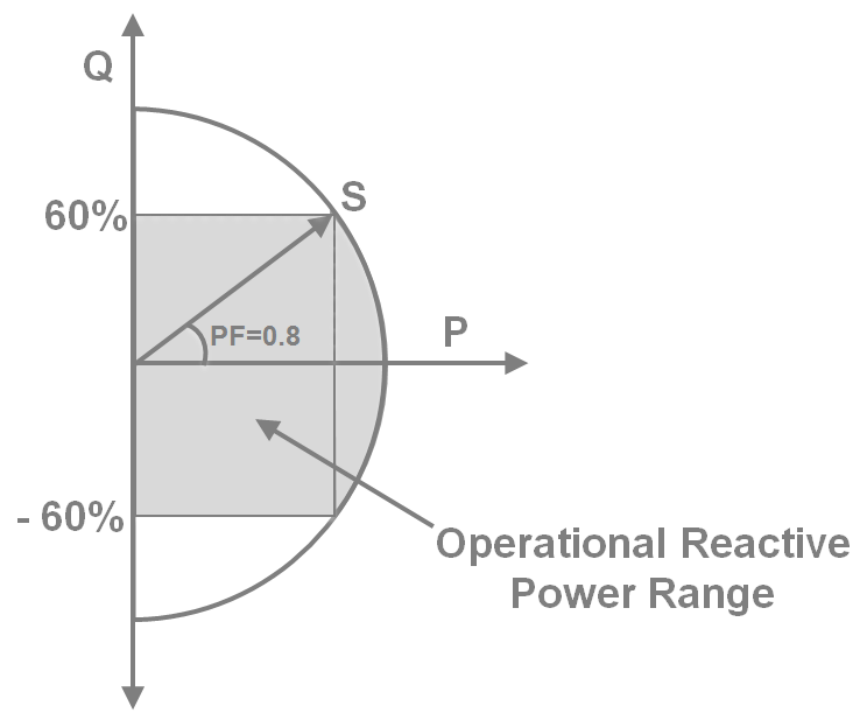

Figure 12. Diagram depicting inverter capacity with reactive power capability range 


\subsubsection{Photovoltaic and Smart Inverter Random Allocation}

Prior to running the annual simulations, the PV penetration and smart inverter density were set by the Python control code. The algorithm ensured that the location of PV and smart inverters in the small penetrations of the study were subsets of the system locations in larger penetrations (e.g. the PV system size and locations included at the $25 \%$ penetration level would also be included as a subset of the PV systems at the $50 \%$ penetration level). This functionality was implemented to ensure that results are consistent across all penetration levels.

To randomly allocate PV systems and smart inverters, each load with a PV system was first given a number in a consecutive series. Then from that set of numbers, the numbers were then moved to a randomized order. An example of the randomized ordering of PV systems is provided in the example below.

- Numbered PV systems: $\{1,2,3,4,5,6,7,8\}$

- $\quad$ Randomized PV systems: $\{2,6,3,7,4,1,5,8\}$

The PV systems to enable were determined by the penetration level and the randomly ordered series of PV systems. For example, for the $25 \%$ PV penetration scenarios, PV systems with indices starting from the first one in the random number series would be enabled until their total ratings reached the $25 \%$ penetration level; or using the example above, if all PV systems are of the same size, systems 2 and 6 would be activated. For 50\% penetration, systems 2, 6, 3, and 7 would be enabled. To assure that the allocation of PVs and smart inverters are consistent across different simulations from multiple threads or computers, this random series was only generated once per distribution system and uploaded to the code repository. It was reused in subsequent simulations across different instances of the code and different computers. The same process using the same random series number was applied to enable smart inverters based on the smart inverter density from the enabled PV systems.

\subsection{Volt-VAR Curve Selection Methodology}

The smart inverter reactive power output follows the VVC. The smart inverter VVC function was selected as it can autonomously respond to the voltage at the inverter terminal. Additionally, the VVC implementation marries well with the goals of a voltage reduction scheme, to flatten and lower the distribution system voltage profile. It is important to note that the smart inverters were operating autonomously, as their output was only dictated by their preset VVC and their local voltage in the model. The InvControl object allowed OpenDSS to determine the outputs of the smart inverters automatically and solved the power flow equations iteratively while updating the smart inverters output to arrive at a converged solution. 
Depending on the purpose of the study, the characteristics of the distribution system, and the operational objectives of the utility, the VVC shape could vary. This study defined the VVC by

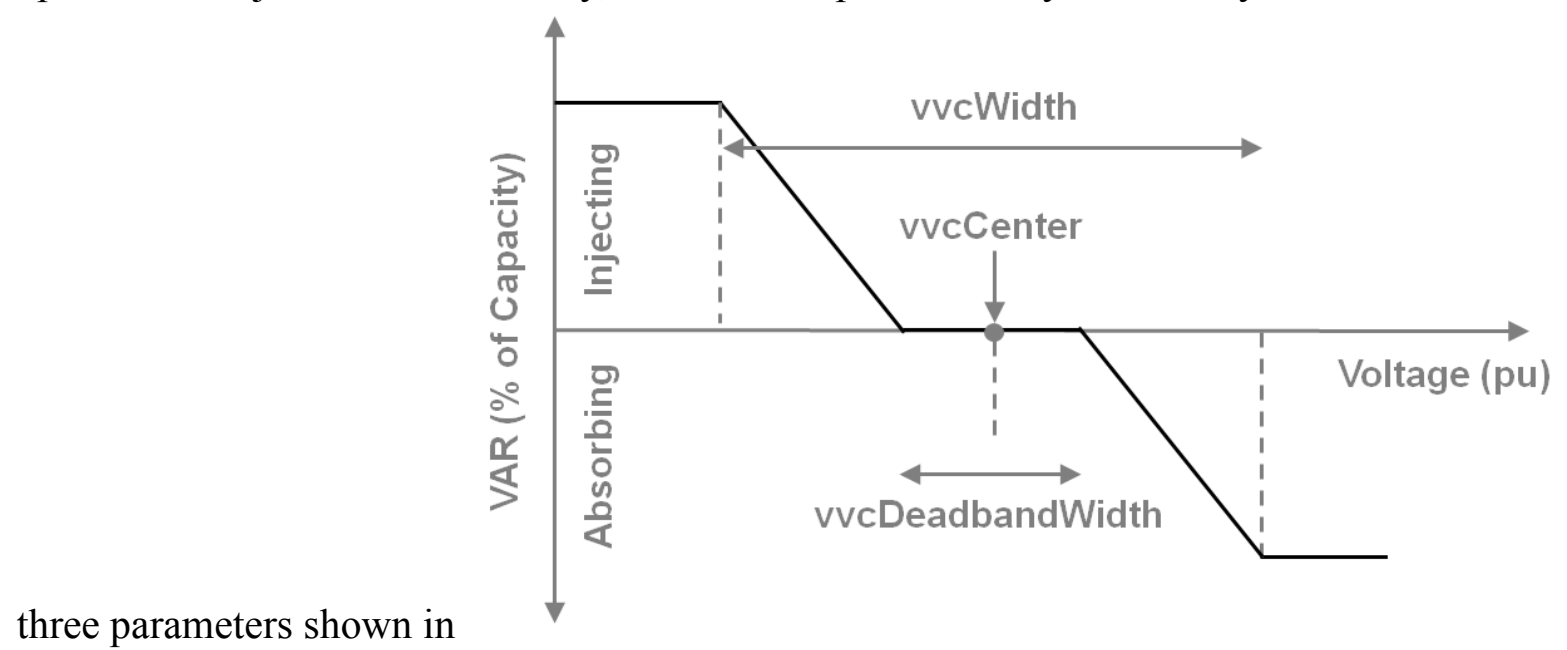

Figure 13, including the VVC center, the VVC voltage width, and the VVC voltage dead band.

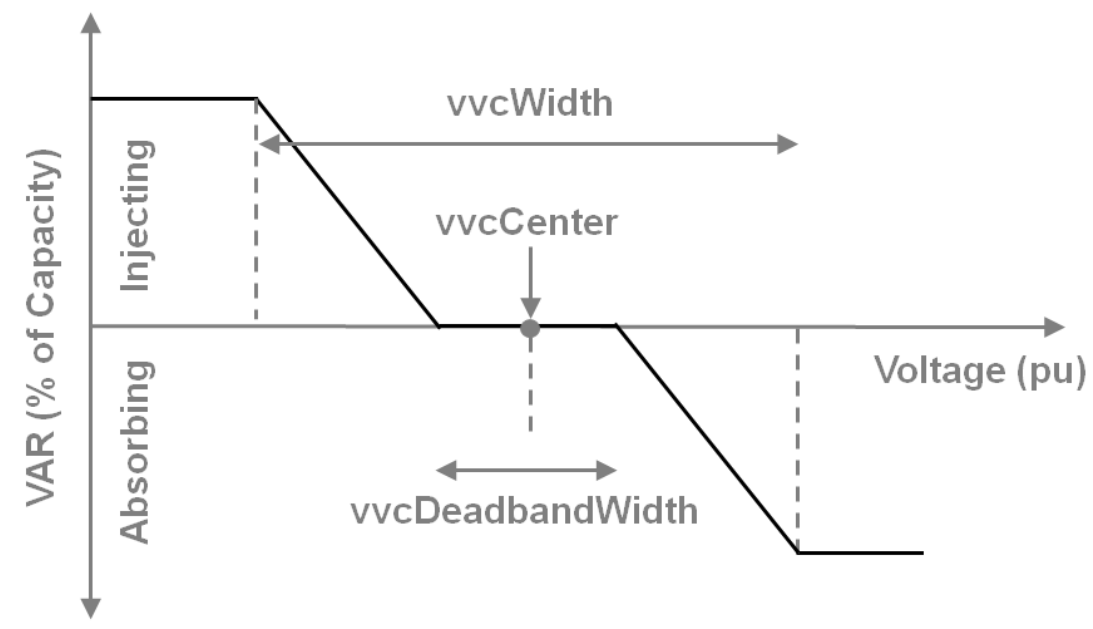

Figure 13. VVC settings with vvcWidth, vvcCenter, and vvcDeadbandWidth. (In this study, the reactive power capability was limited to $60 \%$ of kVA nameplate.)

For this study, the desired voltage of the smart inverters was defined by the VVC center. When the smart inverter voltage was at the VVC center, no VAR support is needed. The VVC width determined the slope of the $\mathrm{VVC}$ and how much reactive power the inverter provided to move the terminal voltage toward the VVC center. The smaller the VVC width, the steeper the slope would be. The dead band defined the voltage range around the VVC center in which the inverter would not provide any reactive power.

The user input the VVC using these parameters and the control code assigned this specified curve to all of the smart inverters that were enabled. The steps for selecting the best VVC among all studied curves were: 
1. Determined the voltage range $\left(V_{\text {range }}\right)$ of the feeder at peak load either via simulation or voltage measurement in the field. The best VVC center would be in the range of 0.95 pu to $\left(0.95+V_{\text {range }}\right)$ pu.

2. Ran simulation for base cases for comparison. The penetration level of the base case was $0 \% \mathrm{PV}$. The time periods ran to determine the best VVC included four weeks representing four different seasons.

3. Determined the best center for the VVC by running simulations for the 4 same weeks with different VVC centers for a particular PV penetration level and SI density. For example, if $V_{\text {range }}=0.05 p u$, a sample set of VVC centers to use could be $0.96 \mathrm{pu}$ to $0.99 \mathrm{pu}$ with $0.01 \mathrm{pu}$ increment. The increment amount could be smaller if more precision is desired. After these simulations, the best VVC center was identified as the one that gives the best output (e.g. CVR energy savings, PQS, etc.) compared to the base case in step 2.

4. Determined the best VVC voltage width using the best VVC center from step 3. A sample starting set of $\mathrm{VVC}$ width could range from $0 \mathrm{pu}$ to $0.08 \mathrm{pu}$ with a $0.01 \mathrm{pu}$ increment. The best VVC width was similarly determined as the VVC that yields the highest score.

5. Determined the best VVC dead band with the best VVC center and width of the previous steps. The VVC dead bands must be less than the VVC voltage width. A sample starting set of dead bands could be 0 pu to 0.01 pu with a 0.001 pu increment. The best VVC dead band was similarly determined as the VVC that yields the best result compared to the base case in step 2.

In this study, two different VVCs were determined for each of the distribution systems studied. One of them was with VO activated and with the objective to increase overall voltage reduction energy savings. The other was without VO activated and with the objective to yield the best PQS. The selected VVCs for HECO and PG\&E feeders are shown in Section 5. 


\section{Case Study}

The CVR VO methodology was applied to two different distribution systems, one from PG\&E and one from HECO. For each system, multiple PV penetrations and smart inverter densities were studied to quantify the impact of distributed PV with smart inverters on voltage reduction energy savings and the PQS.

\subsection{Hawailian Electric Companies' Distribution System}

The HECO distribution system identified for this case study was selected by HECO because it represents an area of high PV penetration, a mix of overhead and underground conductors, and partial commercial loads with residential loads.

The sections below describe the distribution system model and PV and load data, before diving deeper into the annual simulations results.

\subsubsection{Distribution System Model}

Table 4 lists the system characteristics of the HECO distribution system. The peak power of the existing PV penetration is $10.5 \mathrm{MW}$, which is in excess of $300 \%$ of gross day-time minimum load and nearly $200 \%$ of peak load. Voltage is regulated by the LTC on the substation transformer. At the end of the line, the voltage for less than $1 \%$ of the load is regulated by a line regulator. To simplify the analysis, the line regulator was removed from the model with all downstream load aggregated at the primary voltage node upstream of the line regulator. Given that the line regulator only serves $1 \%$ of the load, this simplification should not have a material impact on the results. There are no capacitors connected at the substation or on the distribution system.

Table 4. HECO Distribution System Characteristics

\begin{tabular}{l|c}
\hline Model Property & Value \\
\hline Substation Bank Size & $10 \mathrm{MVA}$ \\
\hline Circuit Primary Voltage & $12 \mathrm{kV}$ \\
\hline Number of Circuits & 2 \\
\hline Peak Bank Load & $6.3 \mathrm{MW}$ \\
\hline Max Circuit Distance & $14.5 \mathrm{~km}$ \\
\hline LTC Settings & 0 \\
\hline Capacitor Banks & Base $=120 \mathrm{~V}, \mathrm{R}=7, \mathrm{X}=7$, CTPrim $=600$ \\
\hline
\end{tabular}

HECO provided the Synergi model of the distribution system that was converted to an OpenDSS model for a detailed analysis. The converted model was first validated by comparing it to the original Synergi model. The verification of the converted OpenDSS model was performed based on the following categories.

1. Topology. Visually inspect and compare the converted model with the original Synergi model.

2. Voltage. After performing a load flow, compare the node voltage between the converted model and the original Synergi model and ensure the voltage difference is less than $1 \%$. 
3. Impedance. After performing a fault study, compare the positive sequence impedances between the converted model and the original Synergi model and ensure the impedance difference is less than $3 \%$.

Figure 14 shows the distribution system topology in Synergi (left) and OpenDSS (right). The line distances and coordinates are appropriately converted and pass visual topology inspection.
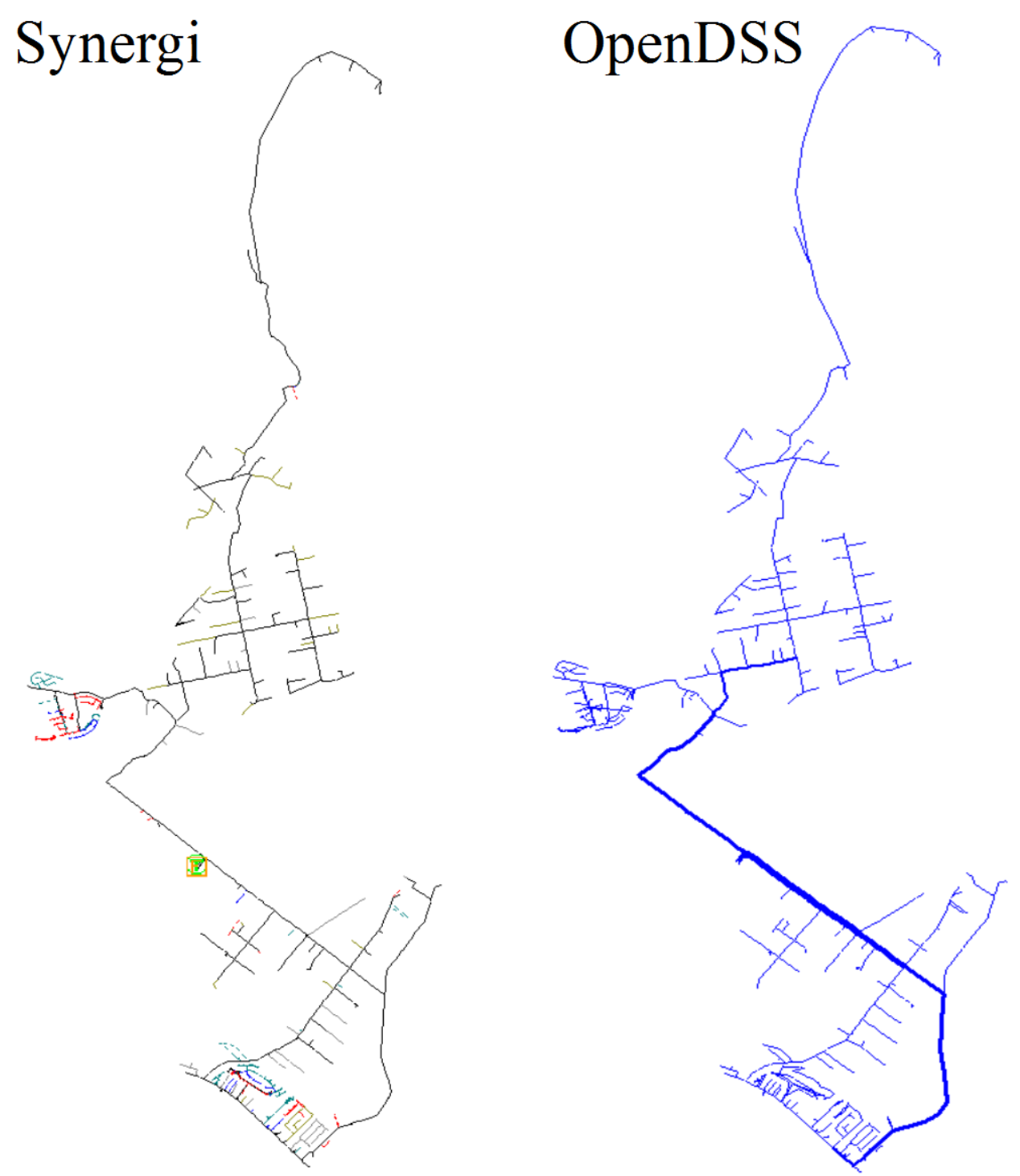

Figure 14. Geographical topology view of the HECO distribution system in Synergi and OpenDSS

Figure 15 shows the voltage profile of the distribution system as well as the voltage error (obtained at full load) as a function of distance. Voltage error distribution is also shown as a histogram. All voltage errors were less than $0.6 \%$. Voltage errors increased toward the end of the feeder. This is typical as errors at the end of the distribution system also include the small errors that occurred closer to the substation. 

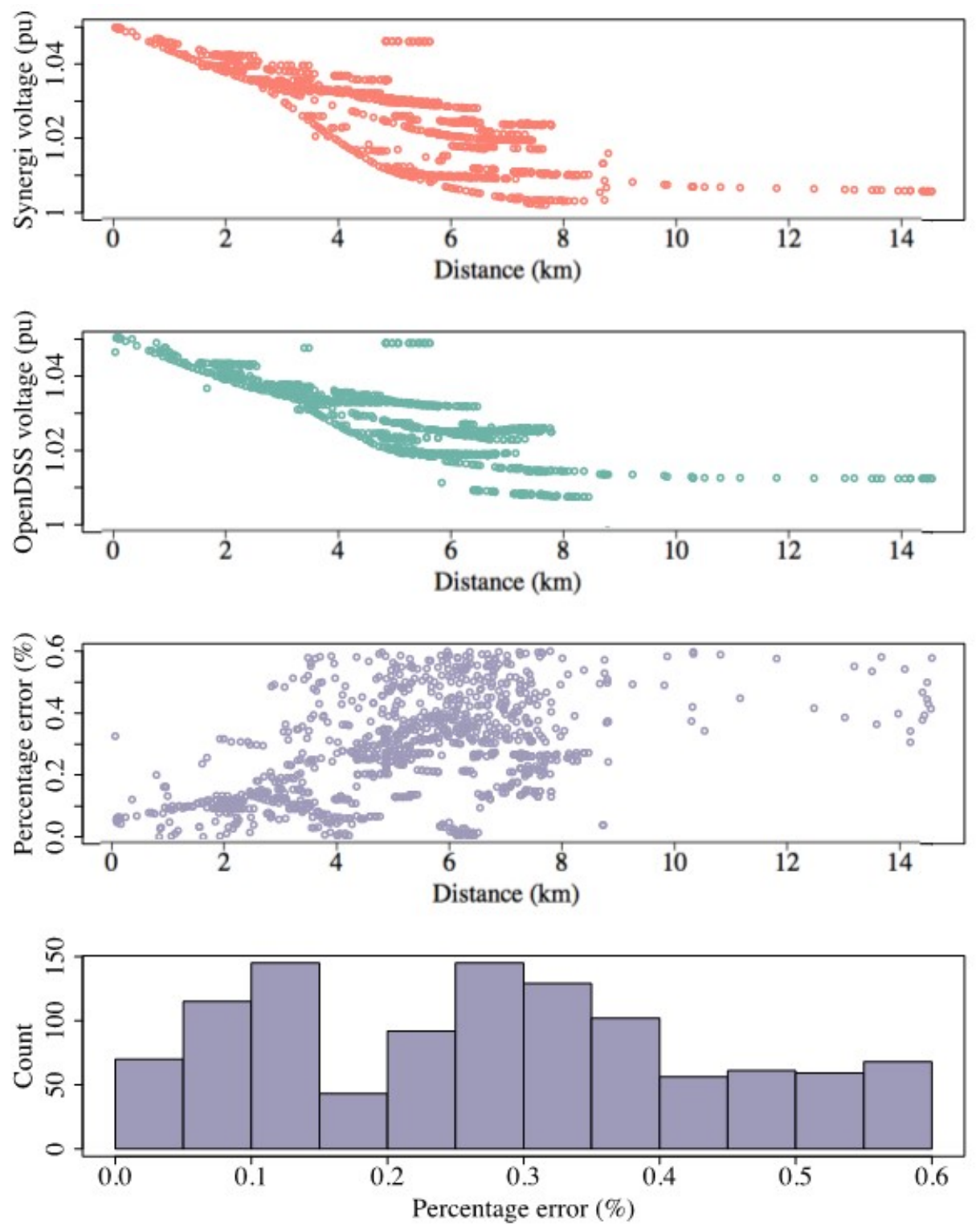

Figure 15. Voltage profile and voltage error of the HECO distribution system

Figure 16 shows the positive sequence impedance profile of the distribution system as well as impedance error as a function of distance. Sequence impedance errors distribution is also shown as a histogram. The maximum sequence impedance errors generally ranged from $0.5 \%$ to $2.5 \%$ with all errors less than $3 \%$.

Based on Figure 14 through Figure 16, it was concluded that the model was accurately converted to OpenDSS. 

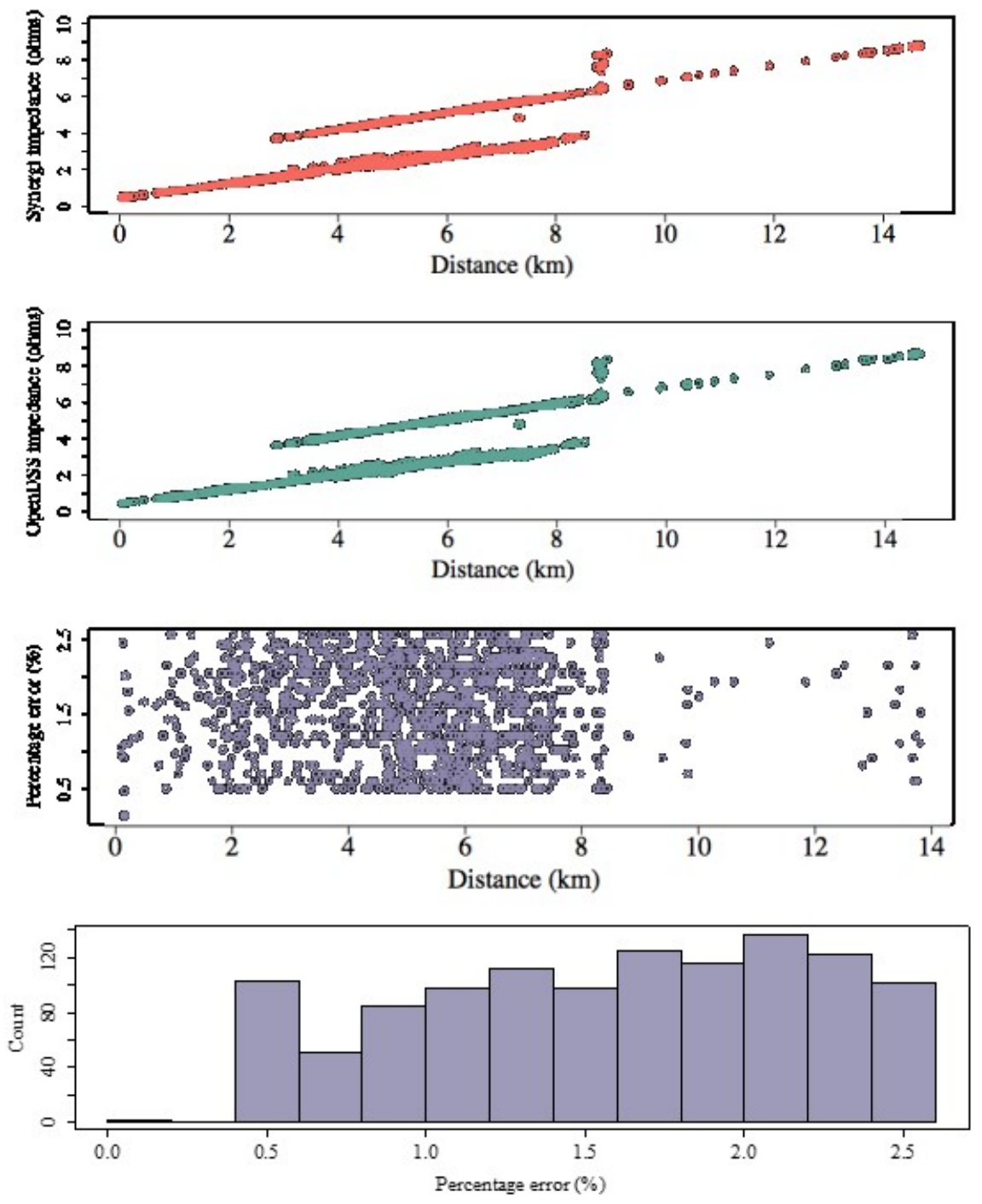

Figure 16. Positive sequence impedance profile and sequence impedance error of the HECO distribution system

The model provided by HECO did not include any secondary, low voltage circuits. These were necessary for the analysis and were added into the validated OpenDSS model. Based on historical load data, HECO provided the distribution of residential and commercial loads at $89 \%$ and $11 \%$ respectively. The customer type was used to determine secondary conductor ratings and lengths. Based on conversations with HECO, the residential loads were assigned a $1 / 0 \mathrm{Al}$ conductor with a secondary spanning $100 \mathrm{ft}$; ; whereas commercial loads were assigned a 4/0 Al conductor with a secondary length of $60 \mathrm{ft}$.

\subsubsection{Annual Photovoltaic and Load Data}

Table 5 summarizes the PV and load data measurements for HECO distribution system, taken from HECO's SCADA system. To capture the accurate PV plant variability on the system and to generate accurate system statistics, a complete 1-minute data set (i.e., 525,600 measurements) for 2015 was used. Specifically, the 1-minute 6 A.M. to 9 P.M. SCADA data was merged with the 
24-hour hourly data, which was linearly interpolated to 1 minute. All missing or out-of-range SCADA values were replaced with a 30-minute running average value before and after the missing data sample.

Table 5. Annual PV and Load Data Summary for the HECO Distribution System

\begin{tabular}{lll}
\hline Measurement Name & Source & Data Resolution \\
\hline Substation Active Power & SCADA Telemetry & $3 \mathrm{sec}-30 \mathrm{sec}$ \\
\hline PV Irradiation & Irradiation Measurement Unit & $1 \mathrm{sec}-60 \mathrm{sec}$ \\
\hline
\end{tabular}

To create "native" substation load (i.e. the true load without PV production's offset), PV production was added to the substation power at each time step. A sample day demonstrating this process is shown in Figure 17. The green line is the measured value with PV production offsetting net load. The red line is the calculated native value with the PV production removed. This data filling, interpolation, and smoothing process created a complete 15-minute data set of the 2015 substation PV and load data that was used as a time-series input for the modeling and analysis. The load and PV profile were applied to each load and PV object in order to perform QSTS simulation.

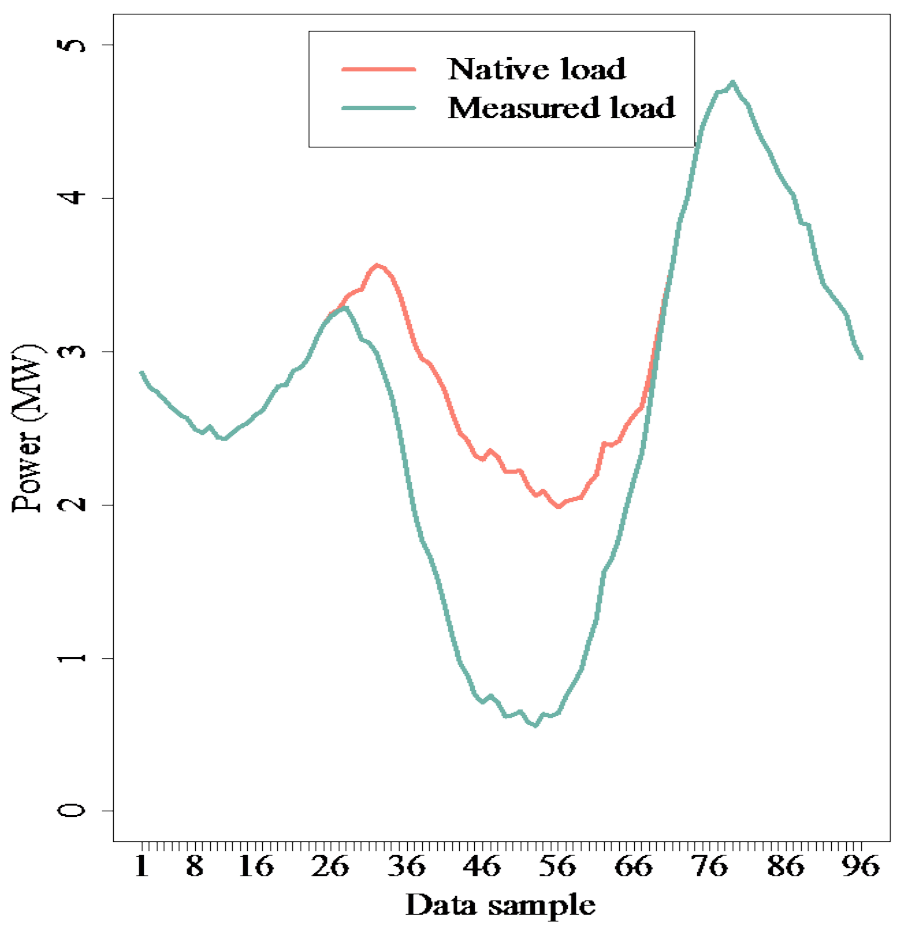

Figure 17. Example illustrating how native load was calculated

\subsubsection{Simulation Results}

This section presents simulation results for the HECO distribution system. Scenarios without PV were first studied to obtain base case results. Following this, multiple scenarios with various PV penetrations and smart inverter densities were studied to evaluate their impact on voltage reduction energy savings and power quality. 


\subsubsection{Base Case}

All PV systems currently existing on the HECO distribution system were first removed from the model before two different scenarios were studied - base case VO without PV. The QSTS simulation was conducted for one year with 8,760 1-hour time steps.

Figure 18 shows the total substation load demand at each time step, with an annual energy consumption of 30,707 MWh. With VO implemented, the annual energy consumption was reduced to $30,245 \mathrm{MWh}$, a $1.505 \%$ reduction in energy.

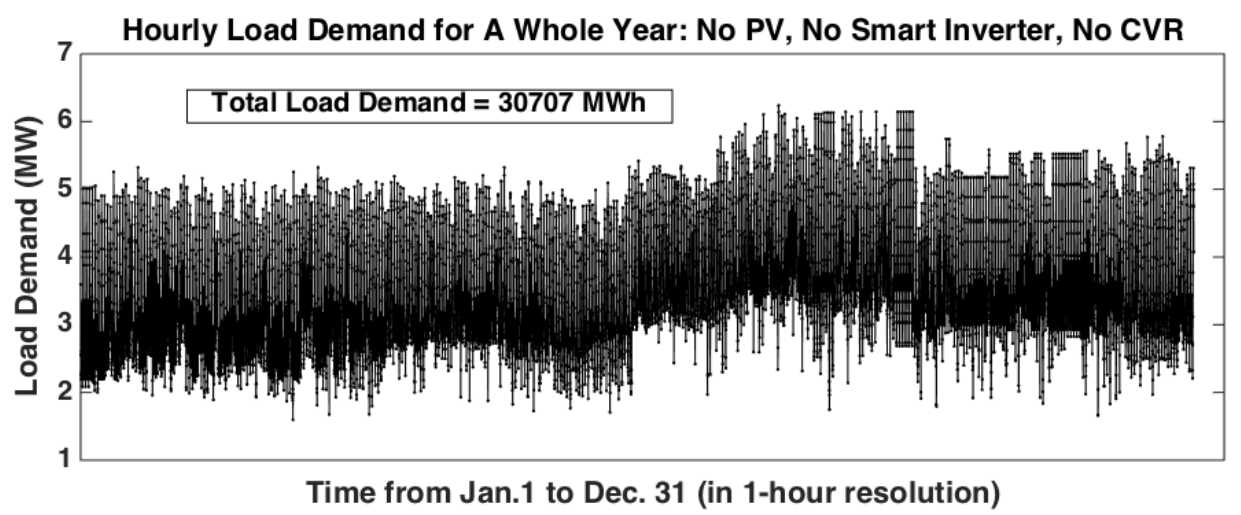

Figure 18. Hourly load demand for the HECO distribution system base case

Figure 19 shows the maximum and minimum system voltages for each time steps with and without VO. There was only a small difference between maximum voltages; however, after implementing VO, the range of minimum voltage is greatly reduced. Before, it ranged widely from 0.949 pu to $0.993 \mathrm{pu}$, but after, the minimum voltage of the system was reduced, staying around $0.955 \mathrm{pu}$ for the entire year. The minimum voltages became flatter after implementing voltage reduction. This is a result of the voltage reduction scheme attempting to lower the voltage as much as possible at each time step in order to make the minimum voltage as close to $0.95 \mathrm{pu}$ as possible without going under.
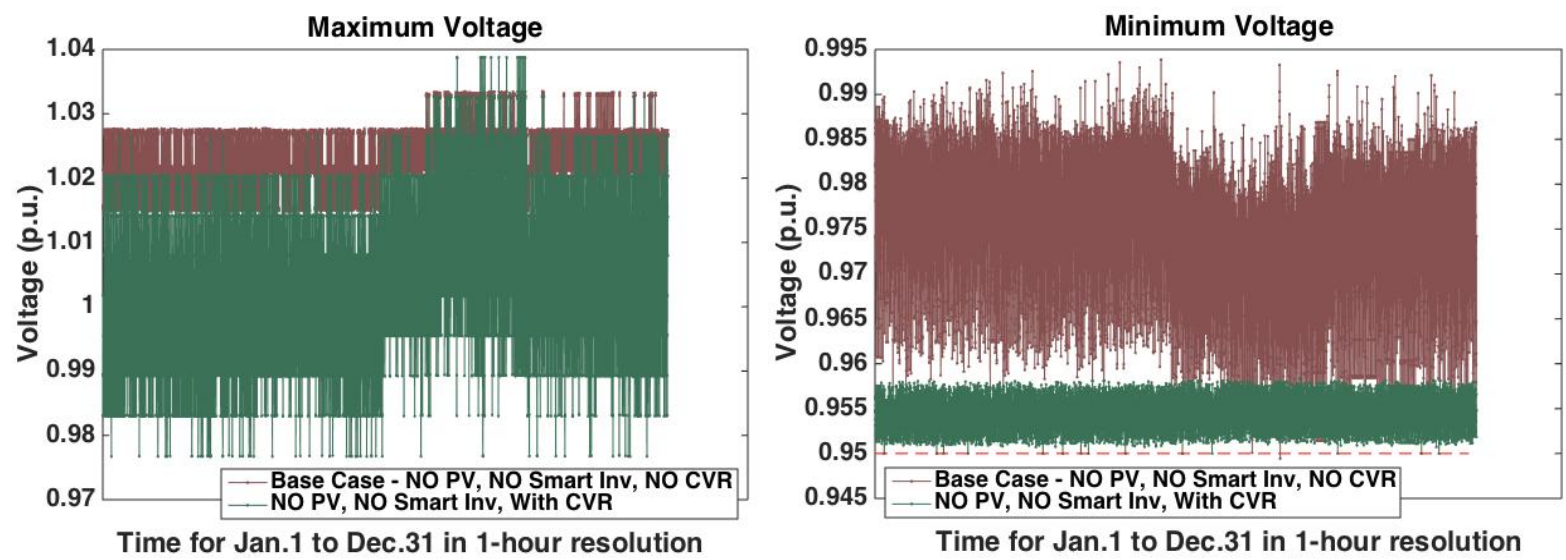

Figure 19. Annual maximum and minimum voltages for the HECO system base case and CVR VO without PV 
Table 6 compares the power quality scores obtained for base case with and without VO implemented. With VO implemented, the PQS was reduced from 9.0516 to 8.2409. For both cases, system voltage magnitudes were always within ANSI limits. Therefore, SAVMVI was always 10 . Power quality scores are presented in two forms - the raw value and the score value after interpolation. A value of 10 represents an ideal case. VO implementation caused the raw value of SAVFI to moderately increase and the raw values of SAVUI and SELI to slightly increase. This led to a lower individual score for SAVFI, SAVUI and SELI. VO implementation caused the LTC to change taps more frequently and increase the raw value of SCDOI, reducing the score of SCDOI by almost half. VO implementation helped reduce the reactive power demand from the substation, so the score of SRPDI increased.

Table 6. Power Quality Scores for Base Case and CVR VO without PV for the HECO Distribution System

\begin{tabular}{|c|c|c|c|c|c|c|c|c|c|}
\hline Sce & ario & & SAVMVI & SAVFI & SAVUI & SRPDI & SELI & SCDOI & $P Q S$ \\
\hline \multirow{4}{*}{$\begin{array}{l}\text { व } \\
\text { O } \\
z\end{array}$} & \multirow{2}{*}{$\begin{array}{l}\text { Base } \\
\text { Case }\end{array}$} & $\begin{array}{c}\text { Raw } \\
\text { Value }\end{array}$ & 0 & 0.0019 & 0.0046 & 0.8183 & 0.0155 & 0.1622 & \multirow[t]{2}{*}{9.0516} \\
\hline & & Score & 10 & 9.3833 & 9.3059 & 8.6362 & 8.2824 & 8.7021 & \\
\hline & \multirow{2}{*}{$\begin{array}{c}\text { CVR } \\
\text { VO }\end{array}$} & $\begin{array}{c}\text { Raw } \\
\text { Value }\end{array}$ & 0 & 0.0035 & 0.0047 & 0.7657 & 0.0157 & 0.7096 & \multirow{2}{*}{8.2409} \\
\hline & & Score & 10 & 8.8498 & 9.2971 & 8.7238 & 8.2512 & 4.3232 & \\
\hline
\end{tabular}

Taking the peak load (August 17) and minimum load (March 30) as examples, Figure 20 shows four voltage profiles for these times with and without VO. VO implementation lowered the voltage profiles as expected. The maximum system voltage was reduced from $1.010 \mathrm{pu}$ to 0.982 $\mathrm{pu}$ at minimum load and was reduced from $1.024 \mathrm{pu}$ to $1.012 \mathrm{pu}$ at peak. The minimum voltages after implementing $\mathrm{VO}$ were near the 0.95 pu control limit during both peak and minimum load days. 


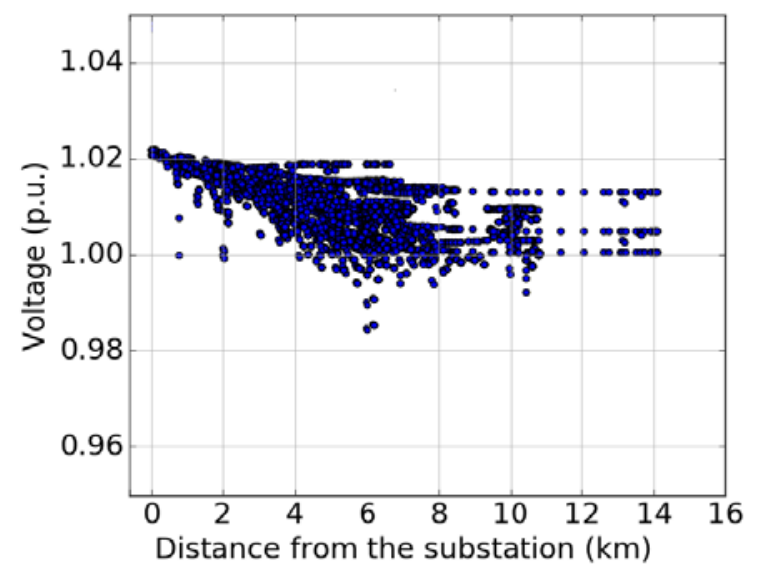

(a) Minimum load without CVR VO

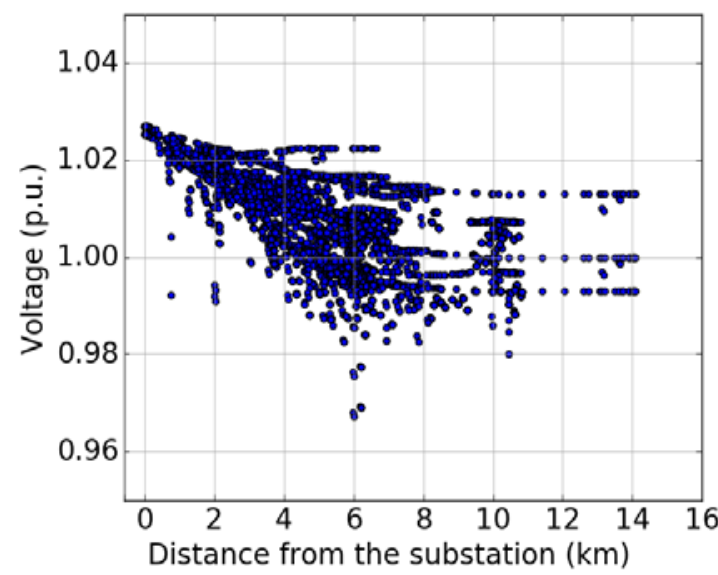

(c) Peak load without CVR VO

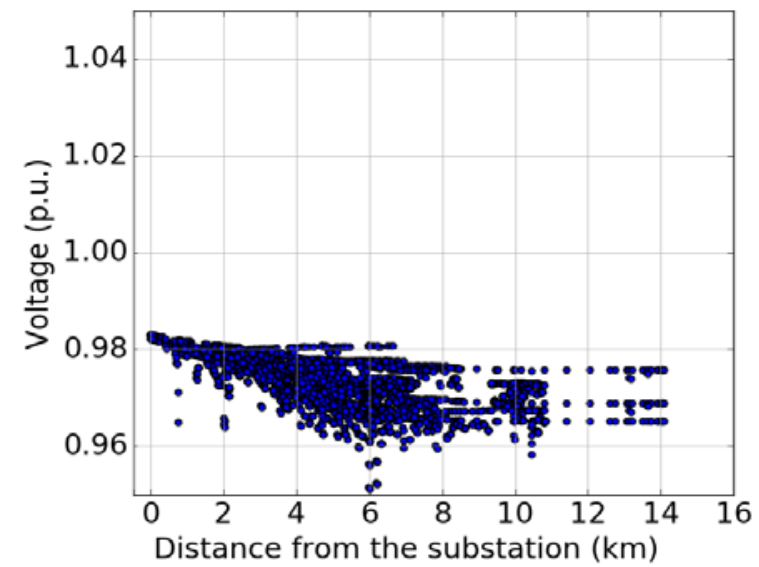

(b) Minimum load with CVR VO

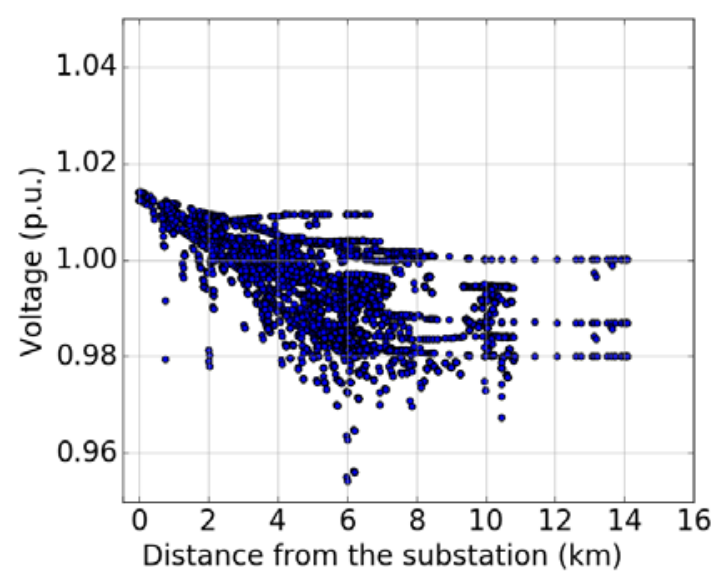

(d) Peak load with CVR Vo

Figure 20. Voltage profiles at peak and minimum load for base case and CVR VO without PV for the HECO distribution system

\subsubsection{Voltage Reduction Energy Savings and Power Quality Score with Photovoltaic and Smart Inverters}

Various scenarios of different PV penetrations and smart inverter densities were studied. PV penetration scenarios included $50 \%, 75 \%, 100 \%, 125 \%$ and $150 \%$ and smart inverter densities included $0 \%, 25 \%, 50 \%, 75 \%$ and $100 \%$. The locations of the PV systems as well as the allocation of smart inverters were randomly selected following the approach given in Section 4.3. Twenty-five (25) different scenarios of PV penetration and smart inverter density were studied with CVR VO implemented.

\subsection{Volt-VAR Curve Selection}

The best VVC for voltage reduction was selected using the approach outlined in Section 4.4. Four weeks, including the first week of January, April, July and October, were studied under $50 \%$ PV penetration and 50\% smart inverter penetration. Table 7 lists all the VVC studied and 
the best VVC parameters selected. The best VVC to achieve the maximum voltage reduction energy savings is shown in Figure 21.

Table 7. VVC Selection for the HECO Distribution System

\begin{tabular}{c|c|c}
\hline Assumptions & Scenarios Studied for VVC Selection & Selected \\
\hline $\begin{array}{c}\text { VVC Width }=0.03 \mathrm{pu} \\
\text { Dead Band Width }=0.008 \mathrm{pu}\end{array}$ & VVC Center $=0.96,0.97,0.98 \mathrm{pu}$ & $0.96 \mathrm{pu}$ \\
\hline $\begin{array}{c}\text { VVC Center }=0.96 \mathrm{pu} \\
\text { Dead Band Width }=0.008 \mathrm{pu}\end{array}$ & VVC Width $=0.01,0.03,0.05,0.07 \mathrm{pu}$ & $0.01 \mathrm{pu}$ \\
\hline $\begin{array}{c}\text { VVC Center }=0.96 \mathrm{pu} \\
\text { VVC Width }=0.01 \mathrm{pu}\end{array}$ & $\begin{array}{c}\text { Dead Band Width }=0,0.002,0.004,0.008,0.01 \\
\mathrm{pu}\end{array}$ & $0.002 \mathrm{pu}$ \\
\hline
\end{tabular}

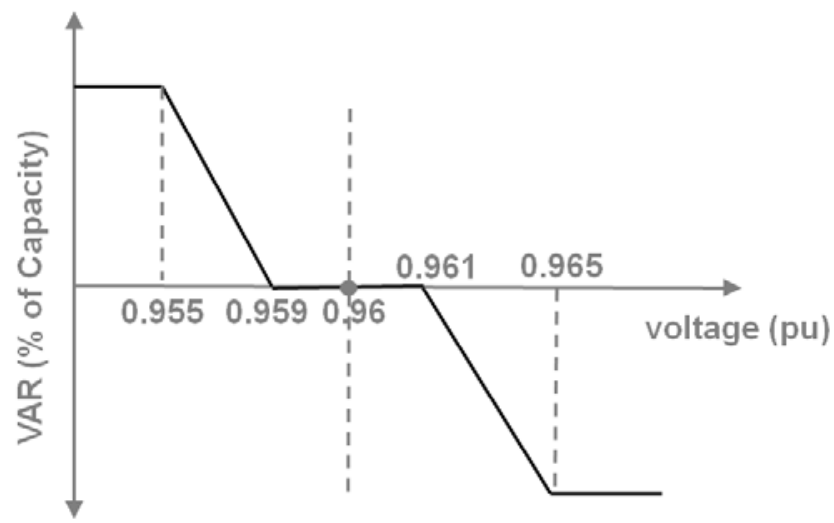

Figure 21. VVC selected to achieve the max voltage reduction energy savings on the HECO distribution system.

(In this study, the reactive power capability was limited to $60 \%$ of kVA nameplate.)

\subsection{Voltage Profile}

Table 8 shows the voltage data summary for all 25 scenarios, including the maximum voltage, minimum voltage, and average voltage of the HECO distribution system, and Figure 22 shows the bar-chart plotting the voltage data. The maximum and minimum voltages were always around 1.0 pu and 0.95 to 0.955 pu respectively. When PV penetration was $50 \%$ or $75 \%$, the greatest voltage reduction effect occurs when smart inverter density was $25 \%$. When PV penetration was $100 \%, 125 \%$ or $150 \%$, the greatest voltage reduction effect occurred when smart inverter density was $100 \%$. Looking at the average voltage results, under the same PV penetration a higher smart inverter density generally helped reduce the voltage profile, and under the same smart inverter density a higher PV penetration also helped reduce the voltage profile in most scenarios. 
Table 8. Voltage Results by PV Penetration and Smart Inverter Density for the HECO Distribution System

\begin{tabular}{c|c|ccc}
\hline \multicolumn{2}{c}{ Scenario } & \multicolumn{3}{c}{ Voltage Result } \\
\hline PV & $\begin{array}{c}\text { Smart } \\
\text { Inverter }\end{array}$ & Maximum & Minimum & Average \\
\hline \multirow{5}{*}{$50 \%$} & 0 & 1.0082 & 0.9543 & 0.9827 \\
& $25 \%$ & 1.0062 & 0.9528 & 0.9798 \\
& $50 \%$ & 1.0096 & 0.9534 & 0.9810 \\
& $75 \%$ & 1.0078 & 0.9533 & 0.9795 \\
& $100 \%$ & 1.0072 & 0.9531 & 0.9787 \\
\hline \multirow{5}{*}{$75 \%$} & 0 & 1.0072 & 0.9543 & 0.9825 \\
& $25 \%$ & 1.0068 & 0.9529 & 0.9792 \\
& $50 \%$ & 1.0089 & 0.9532 & 0.9789 \\
& $75 \%$ & 1.0097 & 0.9531 & 0.9785 \\
& $100 \%$ & 1.0063 & 0.9530 & 0.9771 \\
\hline \multirow{5}{*}{$100 \%$} & 0 & 1.0039 & 0.9543 & 0.9803 \\
& $25 \%$ & 1.0047 & 0.9529 & 0.9773 \\
& $50 \%$ & 1.0043 & 0.9531 & 0.9755 \\
& $75 \%$ & 1.0024 & 0.9532 & 0.9745 \\
& $100 \%$ & 0.9986 & 0.9531 & 0.9713 \\
\hline \multirow{5}{*}{$125 \%$} & 0 & 1.0039 & 0.9542 & 0.9806 \\
& $25 \%$ & 1.0038 & 0.9528 & 0.9761 \\
& $50 \%$ & 1.0055 & 0.9532 & 0.9758 \\
& $75 \%$ & 0.9979 & 0.9531 & 0.9715 \\
& $100 \%$ & 0.9987 & 0.9526 & 0.9709 \\
\hline \multirow{5}{*}{$150 \%$} & 0 & 1.0024 & 0.9541 & 0.9789 \\
& $25 \%$ & 1.0043 & 0.9528 & 0.9753 \\
& $50 \%$ & 1.0039 & 0.9531 & 0.9741 \\
& $75 \%$ & 1.0012 & 0.9529 & 0.9709 \\
& $100 \%$ & 0.9976 & 0.9516 & 0.9689 \\
\hline
\end{tabular}



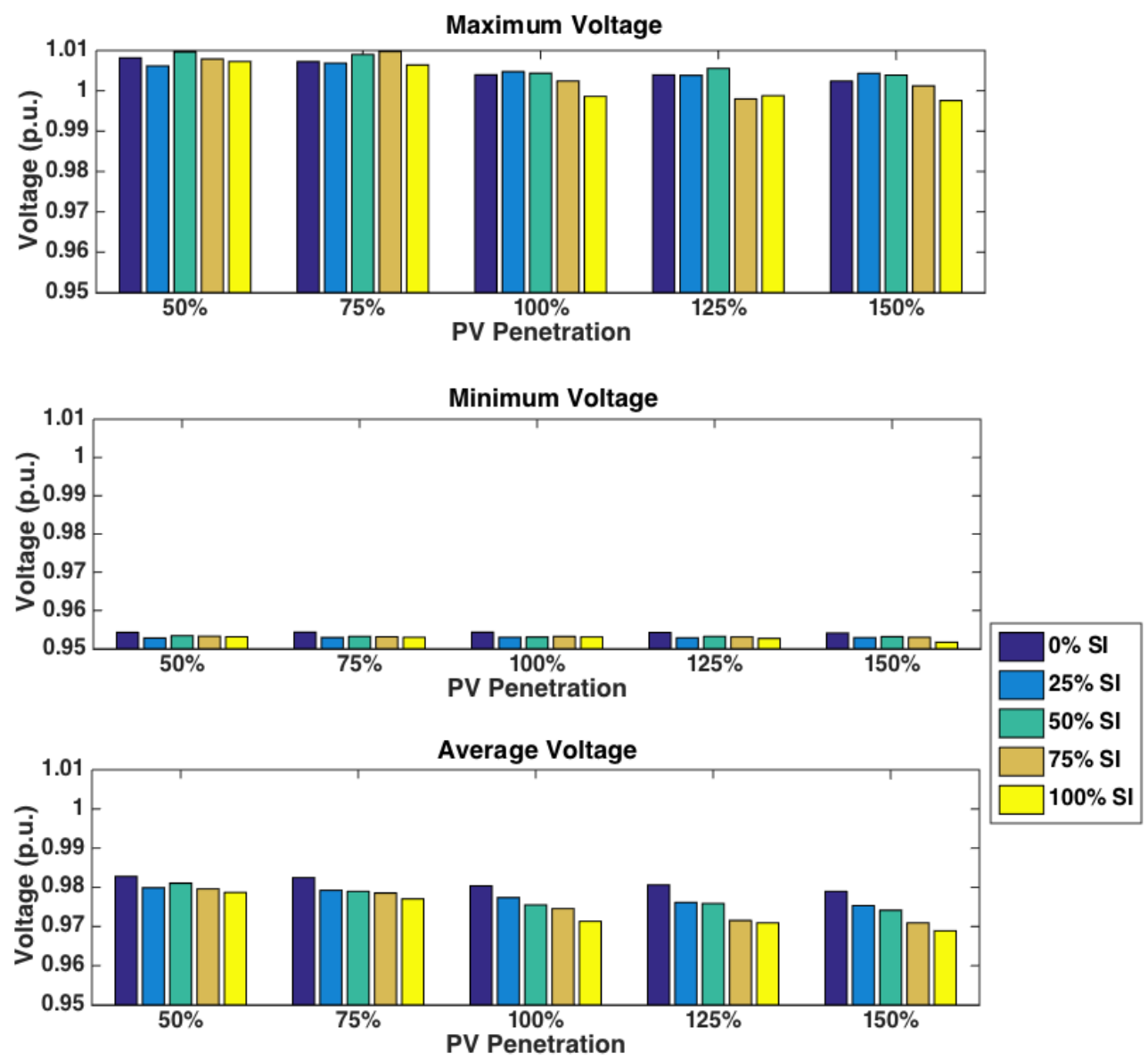

Figure 22. Voltage results for different PV penetrations and smart inverter densities for HECO distribution system

\subsection{Voltage Reduction Benefit}

Table 9 shows voltage reduction energy savings for all 25 scenarios. With only one exception (50\% PV and 50\% SI), a higher smart inverter density with PV penetration remaining constant led to larger energy savings, with the highest voltage reduction savings at $100 \%$ smart inverter penetration (Figure 23). With two exceptions, a higher PV penetration with same smart inverter density led to larger energy savings (Figure 24). 
Table 9. Voltage Reduction Energy Savings by PV Penetration and Smart Inverter Density for the HECO Distribution System ${ }^{1}$

\begin{tabular}{c|c|c|c|c|c|c|}
\hline \multicolumn{2}{|c|}{$\begin{array}{c}\text { Voltage Reduction } \\
\text { Energy Savings (\%) }\end{array}$} & \multicolumn{5}{|c|}{ Smart Inverter Density } \\
\cline { 3 - 7 } & $\mathbf{0 0 \%}$ & 1.59 & 1.89 & 1.81 & 1.95 & 2.05 \\
\hline \multirow{4}{*}{$\begin{array}{c}\text { PV } \\
\text { Penetration }\end{array}$} & $\mathbf{7 5 \%}$ & 1.59 & 1.92 & 1.97 & 2.03 & 2.23 \\
\cline { 3 - 7 } & $\mathbf{1 0 0 \%}$ & 1.74 & 2.04 & 2.23 & 2.38 & 2.70 \\
\cline { 3 - 7 } & $\mathbf{1 2 5 \%}$ & 1.71 & 2.15 & 2.21 & 2.68 & 2.73 \\
\cline { 3 - 7 } & $\mathbf{1 5 0 \%}$ & 1.82 & 2.18 & 2.38 & 2.69 & 2.88 \\
\hline
\end{tabular}

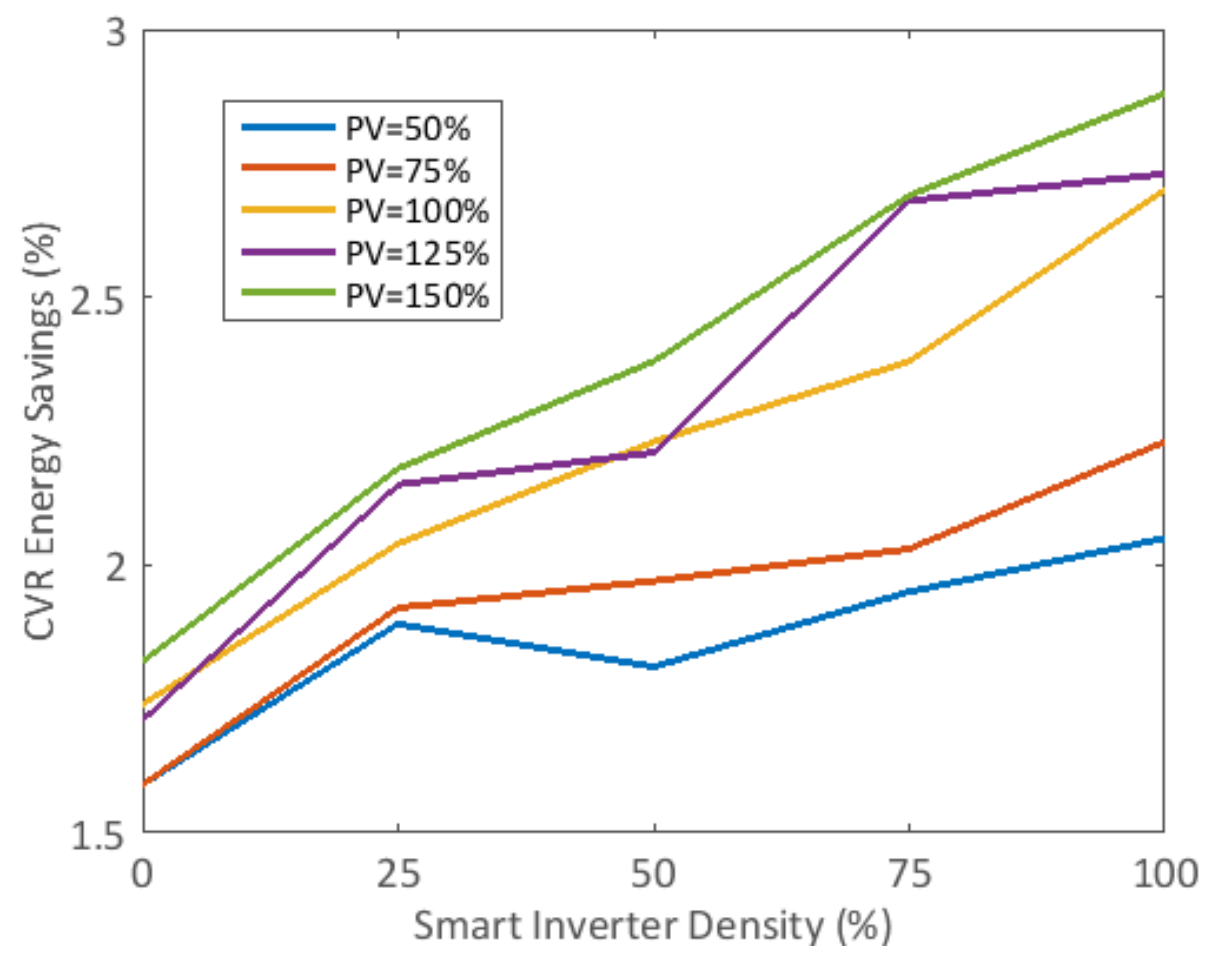

Figure 23. Voltage reduction energy savings as a function of smart inverter density at different PV penetrations for the HECO distribution system

\footnotetext{
${ }^{1}$ These savings were based on modeling the loads with a CVR factor of 0.8 . If the CVR factor for the load type studied is known to be higher or lower, an approximately linear increase or decrease in savings is expected.
} 


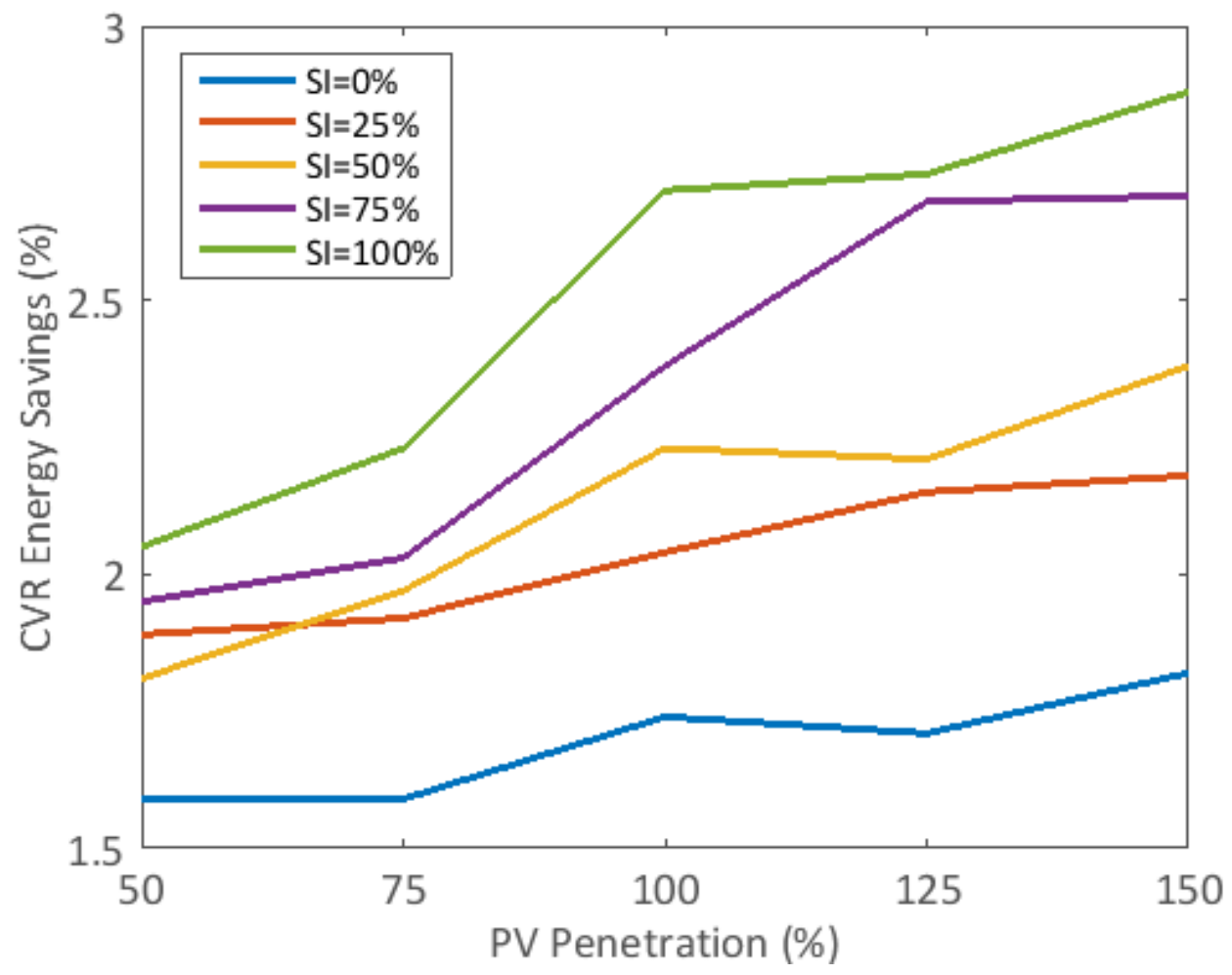

Figure 24. Voltage reduction energy savings as a function of PV penetration at different smart inverter densities for the HECO distribution system

\subsection{Power Quality Score}

Table 10 and Table 11 show the results of the PQS for all 25 scenarios, with the raw values and interpolated scores respectively. Table 12 shows a summary of the combined PQS. Compared with the result in Table 6, the combination of VO being active and smart inverter volt-VAR control reduced the score for SCDOI and SRPDI in order to increase energy savings at the load nodes. This indicates more regulator tap changes and increased reactive power demand. The increased reactive power demand occurred because the volt-VAR control of the smart inverter absorbs VAR to lower voltage, leading to a lower SRPDI score. With this voltage reduction, more regulator tap changes have to occur to lower the voltage. These lower scores were a result of this iteration of the voltage reduction implementation and different results would most likely occur with a more sophisticated voltage reduction control approach that optimizes for reactive power demand and voltage reduction. Further, with VO being active, it was difficult to differentiate PV penetration's and smart inverter density's impact vs. the impact of voltage reduction on the power quality score. 
Table 10. Power Quality Score Raw Values by PV Penetration and Smart Inverter Density for the HECO Distribution System

\begin{tabular}{c|c|cccccc}
\hline \multicolumn{2}{c|}{ Scenario } & \multicolumn{5}{c}{ Power Quality Metric Value } \\
\hline PV & $\begin{array}{c}\text { Smart } \\
\text { Inverter }\end{array}$ & SAVMVI & SAVFI & SAVUI & SRPDI & SELI & SCDOI \\
\hline \multirow{5}{*}{$50 \%$} & 0 & 0 & 0.003563 & 0.004316 & 0.74660 & 0.013216 & 0.75441 \\
& $25 \%$ & 0 & 0.003357 & 0.005013 & 1.23789 & 0.014858 & 0.76163 \\
& $50 \%$ & 0 & 0.003550 & 0.006446 & 1.55127 & 0.015962 & 0.81067 \\
& $75 \%$ & 0 & 0.003211 & 0.006399 & 1.84827 & 0.017280 & 0.82615 \\
& $100 \%$ & 0 & 0.002987 & 0.005260 & 2.08587 & 0.018327 & 0.83595 \\
\hline \multirow{5}{*}{$75 \%$} & 0 & 0 & 0.003637 & 0.004034 & 0.74306 & 0.012609 & 0.81055 \\
& $25 \%$ & 0 & 0.003748 & 0.005847 & 1.43152 & 0.014938 & 0.86584 \\
& $50 \%$ & 0 & 0.003220 & 0.006403 & 1.88596 & 0.016863 & 0.87832 \\
& $75 \%$ & 0 & 0.003149 & 0.005468 & 2.28489 & 0.018818 & 0.93934 \\
& $100 \%$ & 0 & 0.002962 & 0.002559 & 2.68218 & 0.020968 & 0.94860 \\
\hline \multirow{5}{*}{$100 \%$} & 0 & 0 & 0.003967 & 0.003989 & 0.73866 & 0.012629 & 0.84793 \\
& $25 \%$ & 0 & 0.004041 & 0.005781 & 1.46985 & 0.015085 & 0.93988 \\
& $50 \%$ & 0 & 0.003587 & 0.004696 & 1.94569 & 0.017148 & 0.97198 \\
& $75 \%$ & 0 & 0.003398 & 0.002383 & 2.48669 & 0.020058 & 1.03044 \\
& $100 \%$ & 0 & 0.004180 & 0.002451 & 2.60239 & 0.020573 & 1.04674 \\
\hline \multirow{5}{*}{$125 \%$} & 0 & 0 & 0.004129 & 0.003753 & 0.74062 & 0.012803 & 0.85410 \\
& $25 \%$ & 0 & 0.004005 & 0.005111 & 1.61397 & 0.015893 & 0.93300 \\
& $50 \%$ & 0 & 0.003616 & 0.004634 & 2.17136 & 0.018517 & 1.01333 \\
& $75 \%$ & 0 & 0.004197 & 0.002387 & 2.60819 & 0.020740 & 1.04097 \\
& $100 \%$ & 0 & 0.004658 & 0.002926 & 2.91063 & 0.022479 & 1.17817 \\
\hline \multirow{5}{*}{$150 \%$} & 0 & 0 & 0.004240 & 0.003784 & 0.74205 & 0.01361 & 0.98820 \\
& $25 \%$ & 0 & 0.003907 & 0.005064 & 1.67162 & 0.01701 & 1.09688 \\
& $50 \%$ & 0 & 0.003385 & 0.002351 & 2.35426 & 0.02058 & 1.16262 \\
& $75 \%$ & 0 & 0.004379 & 0.002701 & 2.57812 & 0.02163 & 1.22365 \\
& $100 \%$ & 0 & 0.004735 & 0.003846 & 3.09897 & 0.02516 & 1.46314 \\
\hline
\end{tabular}


Table 11. Power Quality Score by PV Penetration and Smart Inverter Density for the HECO Distribution System

\begin{tabular}{c|c|ccccccc}
\hline \multicolumn{7}{c|}{ Scenario } & \multicolumn{7}{c}{ Individual Score for Each Power Quality Metric } & \multirow{2}{*}{ PQS } \\
\cline { 1 - 7 } PV & $\begin{array}{c}\text { Smart } \\
\text { Inverter }\end{array}$ & SAVMVI & SAVFI & SAVUI & SRPDI & SELI & SCDOI & \\
\hline \multirow{3}{*}{$50 \%$} & 0 & 10 & 8.8121 & 9.3525 & 8.7557 & 8.5316 & 3.9647 & 8.2361 \\
& $25 \%$ & 10 & 8.8809 & 9.2481 & 7.9368 & 8.3491 & 3.9069 & 8.0536 \\
& $50 \%$ & 10 & 8.8164 & 9.0331 & 7.4145 & 8.2264 & 3.5146 & 7.8342 \\
& $75 \%$ & 10 & 8.9296 & 9.0401 & 6.9195 & 8.0800 & 3.3908 & 7.7267 \\
& $100 \%$ & 10 & 9.0043 & 9.2110 & 6.5235 & 7.9637 & 3.3124 & 7.6691 \\
\hline \multirow{5}{*}{$75 \%$} & 0 & 10 & 8.7877 & 9.3949 & 8.7616 & 8.5990 & 3.5156 & 8.1764 \\
& $25 \%$ & 10 & 8.7505 & 9.1230 & 7.6141 & 8.3402 & 3.0732 & 7.8168 \\
& $50 \%$ & 10 & 8.9265 & 9.0396 & 6.8567 & 8.1263 & 2.9735 & 7.6538 \\
& $75 \%$ & 10 & 8.9504 & 9.1797 & 6.1918 & 7.9090 & 2.4853 & 7.4527 \\
& $100 \%$ & 10 & 9.0126 & 9.6162 & 5.5297 & 7.6701 & 2.4112 & 7.3733 \\
\hline $100 \%$ & 0 & 10 & 8.6774 & 9.4016 & 8.7689 & 8.5967 & 3.2165 & 8.1101 \\
& $25 \%$ & 10 & 8.6530 & 9.1328 & 7.5503 & 8.3239 & 2.4809 & 7.6901 \\
& $50 \%$ & 10 & 8.8041 & 9.2955 & 6.7572 & 8.0946 & 2.2241 & 7.5292 \\
& $75 \%$ & 10 & 8.8672 & 9.6425 & 5.8555 & 7.7713 & 1.7565 & 7.3155 \\
& $100 \%$ & 10 & 8.6063 & 9.6323 & 5.6627 & 7.7141 & 1.6260 & 7.2069 \\
\hline $125 \%$ & 0 & 10 & 8.6234 & 9.4369 & 8.7656 & 8.5774 & 3.1672 & 8.0951 \\
& $25 \%$ & 10 & 8.6648 & 9.2333 & 7.3100 & 8.2340 & 2.5360 & 7.6630 \\
& $50 \%$ & 10 & 8.7944 & 9.3048 & 6.3811 & 7.9425 & 1.8933 & 7.3860 \\
& $75 \%$ & 10 & 8.6007 & 9.6418 & 5.6530 & 7.6955 & 1.6722 & 7.2105 \\
& $100 \%$ & 10 & 8.4472 & 9.5611 & 5.1489 & 7.5023 & 0.5746 & 6.8724 \\
\hline $150 \%$ & 0 & 10 & 8.5864 & 9.4325 & 8.7632 & 8.4877 & 2.094 & 7.8940 \\
& $25 \%$ & 10 & 8.6974 & 9.2404 & 7.2140 & 8.1102 & 1.2249 & 7.4145 \\
& $50 \%$ & 10 & 8.8713 & 9.6473 & 6.0762 & 7.7133 & 0.6990 & 7.1679 \\
& $75 \%$ & 10 & 8.5403 & 9.5949 & 5.7031 & 7.5970 & 0.2731 & 6.9514 \\
& $100 \%$ & 10 & 8.4215 & 9.4230 & 4.8351 & 7.2039 & 0 & 6.6473 \\
\hline
\end{tabular}

Table 12. Power Quality Score Summary for the HECO Distribution System

\begin{tabular}{|c|c|c|c|c|c|c|}
\hline \multirow{2}{*}{\multicolumn{2}{|c|}{$P Q S$}} & \multicolumn{5}{|c|}{ Smart Inverter Density } \\
\hline & & $0 \%$ & $25 \%$ & $50 \%$ & $75 \%$ & $100 \%$ \\
\hline \multirow{5}{*}{$\begin{array}{c}\text { PV } \\
\text { Penetration }\end{array}$} & $50 \%$ & 8.24 & 8.18 & 8.11 & 8.10 & 7.89 \\
\hline & $75 \%$ & 8.05 & 7.82 & 7.69 & 7.66 & 7.41 \\
\hline & $100 \%$ & 7.83 & 7.65 & 7.53 & 7.39 & 7.17 \\
\hline & $125 \%$ & 7.73 & 7.45 & 7.32 & 7.21 & 6.95 \\
\hline & $150 \%$ & 7.67 & 7.37 & 7.21 & 6.87 & 6.65 \\
\hline
\end{tabular}

\subsubsection{Power Quality Score without CVR VO}

The previous simulation results showed that VO can help save energy, but may come at the expense of power quality, depending on the VO implementation. In order to assess PV penetration's and smart inverter density's impact on PQS, absent VO, more simulation scenarios were studied. 
The purpose of this additional study was to investigate how smart inverters can improve distribution system power quality. The previous VVC was selected to achieve maximum voltage reduction energy savings only. In this additional study, various VVC centers, including 0.99 , $1.00,1.01$ and $1.02 \mathrm{pu}$, were analyzed to find a new VVC that could achieve the highest power quality score. The result is given in Figure 25. Compared with the no PV base case, the scenarios of $1.00,1.01$ and $1.02 \mathrm{pu}$ all had higher power quality scores, with $1.01 \mathrm{pu}$ at the highest. This new curve center of 1.01 pu was used for all new scenarios.

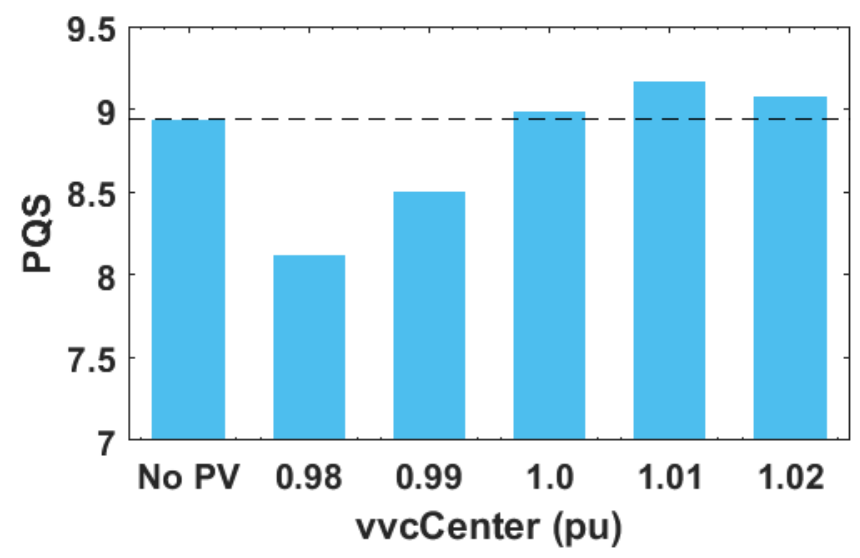

Figure 25. PQS results with different VVC centers for the HECO distribution system

With the changes to the VVC, Table 13 shows the power quality score raw values and Table 14 shows the power quality scores for three PV penetrations, each of which has two different smart inverter penetrations. Without smart inverters, a higher PV penetration caused more voltage fluctuation and voltage regulator tap changes when compared to the base case in Table 6 and the individual scores of SAVFI and SCDOI decreased. However, voltage unbalance, reactive power demand, and system losses improved with increasing PV penetration and the individual scores of SAVUI, SRPDI, and SELI increased. SAVMVI remained at a perfect 10 across all scenarios, signifying no steady-state overvoltage or undervoltage issues. With smart inverters, all metrics except SCDOI generally showed a power quality improvement over the base case in Table 6, indicating that distributed PV with smart inverter can improve power quality.

The decrease in the metric for SCDOI could possibly be improved under different LTC settings. The current settings were not made for all of the PV penetration and smart inverter scenarios studied. The time left in the project did not allow running a sensitivity analysis on the LTC settings, but there was potential to demonstrate a power quality improvement over base case across all PV penetration scenarios studied. 
Table 13. Power Quality Score Raw Values without CVR VO for the HECO Distribution System

\begin{tabular}{c|c|cccccc}
\hline \multicolumn{2}{c|}{ Scenario } & \multicolumn{5}{c}{ Power Quality Metric Value } \\
\hline \multirow{2}{*}{ PV } & $\begin{array}{c}\text { Smart } \\
\text { Inverter }\end{array}$ & SAVMVI & SAVFI & SAVUI & SRPDI & SELI & \multirow{2}{*}{ SCDOI } \\
\hline \multirow{2}{*}{$50 \%$} & 0 & 0 & 0.002090 & 0.004271 & 0.802955 & 0.012999 & 0.248728 \\
& $100 \%$ & 0 & 0.001000 & 0.001386 & 0.349806 & 0.013024 & 0.250100 \\
\hline \multirow{2}{*}{$100 \%$} & 0 & 0 & 0.002236 & 0.003950 & 0.797999 & 0.012365 & 0.385396 \\
& $100 \%$ & 0 & 0.001489 & 0.001654 & 0.991759 & 0.015882 & 0.446885 \\
\hline \multirow{2}{*}{$150 \%$} & 0 & 0 & 0.002512 & 0.003723 & 0.801740 & 0.013174 & 0.532559 \\
& $100 \%$ & 0 & 0.002813 & 0.002668 & 0.438742 & 0.013681 & 0.532559 \\
\hline
\end{tabular}

Table 14. Power Quality Score without CVR VO for the HECO Distribution System

\begin{tabular}{c|c|ccccccc}
\hline \multicolumn{6}{c|}{ Scenario } & \multicolumn{7}{c}{ Individual Score for Each Power Quality Metric } & \multirow{2}{*}{ PQS } \\
\cline { 1 - 7 } PV & $\begin{array}{c}\text { Smart } \\
\text { Inverter }\end{array}$ & SAVMVI & SAVFI & SAVUI & SRPDI & SELI & SCDOI & \\
\hline \multirow{2}{*}{$50 \%$} & 0 & 10 & 9.3030 & 9.3594 & 8.6617 & 8.5556 & 8.0102 & 8.9817 \\
& $100 \%$ & 10 & 9.6658 & 9.7922 & 9.4170 & 8.5529 & 7.9992 & 9.2378 \\
\hline \multirow{2}{*}{$100 \%$} & 0 & 10 & 9.2548 & 9.4074 & 8.6700 & 8.6262 & 6.9168 & 8.8125 \\
& $100 \%$ & 10 & 9.5037 & 9.7519 & 8.3471 & 8.2353 & 6.4249 & 8.7105 \\
\hline \multirow{2}{*}{$150 \%$} & 0 & 10 & 9.1628 & 9.4415 & 8.6638 & 8.5362 & 5.7385 & 8.5906 \\
& $100 \%$ & 10 & 9.0622 & 9.5999 & 9.2688 & 8.4799 & 5.7395 & 8.6917 \\
\hline
\end{tabular}

\subsection{Pacific Gas and Electric Company's Distribution System}

The PG\&E distribution system used for this case study was selected by PG\&E. The sections below describe the distribution system model and PV and load data, before diving deeper into the annual simulation results.

\subsubsection{Distribution System Model}

Table 15 lists system information of the PG\&E distribution system. The PG\&E distribution system model began at the secondary-side of the 45 MVA substation transformer feeding three $21 \mathrm{kV}$ circuits. The topology of the three $21 \mathrm{kV}$ circuits is shown in Figure 26. One LTC was modeled with line-drop compensation regulating the voltage for all three circuits. The three circuits extended through to the end of the low voltage secondary system. The low voltage secondary system was added to the model for the purposes of this case study. 
Table 15. PG\&E Test Distribution System Information

\begin{tabular}{l|c}
\hline Model Property & Value \\
\hline Substation Bank Size & $45 \mathrm{MVA}$ \\
\hline Circuit Primary Voltage & $21 \mathrm{kV}$ \\
\hline Number of Circuits & 3 \\
\hline Peak Bank Load & $37.09 \mathrm{MW}$ \\
\hline Max Circuit Distance & $6.63 \mathrm{miles}$ \\
\hline Total Primary Circuit Miles & $85.65 \mathrm{miles}$ \\
\hline LTC Settings & Base $=122 \mathrm{~V}, \mathrm{R}=4, \mathrm{X}=0$, CTPrim $=1500$ \\
\hline Capacitor Banks & $7(12 \mathrm{MVAR}$ total $)$ \\
\hline
\end{tabular}

The distribution system included various customer types with a mix of urban and rural loads. The circuit was mostly residential at $93.6 \%$ of the customer count and $63.7 \%$ of the peak load. Information on the other customer types can be found in Table 16.

Table 16. Customer Information for PG\&E System Model

\begin{tabular}{c|c|c}
\hline Customer Type & Count & \% Peak Load \\
\hline Residential & 5981 & $63.70 \%$ \\
\hline Commercial & 286 & $13.48 \%$ \\
\hline Industrial & 86 & $18.97 \%$ \\
\hline Agricultural & 13 & $3.04 \%$ \\
\hline Other & 22 & $0.81 \%$ \\
\hline Total & $\mathbf{6 3 8 8}$ & \\
\hline
\end{tabular}

PG\&E provided the CYMDIST model of the PG\&E distribution system that was converted to an OpenDSS model for a detailed analysis. The converted model was first validated by comparing it to the original CYMDIST model. The verification of the converted OpenDSS model was performed based on the following categories.

1. Topology. Visually inspect and compare the converted model with the original CYMDIST model.

2. Current. After performing a load flow, compare the node currents between the converted model and the original CYMDIST model and ensure the current difference is less than $3 \%$.

3. Impedance. After performing a fault study, compare the positive sequence impedances between the converted model and the original CYMDIST model and ensure the impedance difference is less than $3 \%$.

Figure 26 shows the distribution system topology in CYMDIST (left) and OpenDSS (right). The line distances and coordinates were appropriately converted and passed visual topology inspection. 

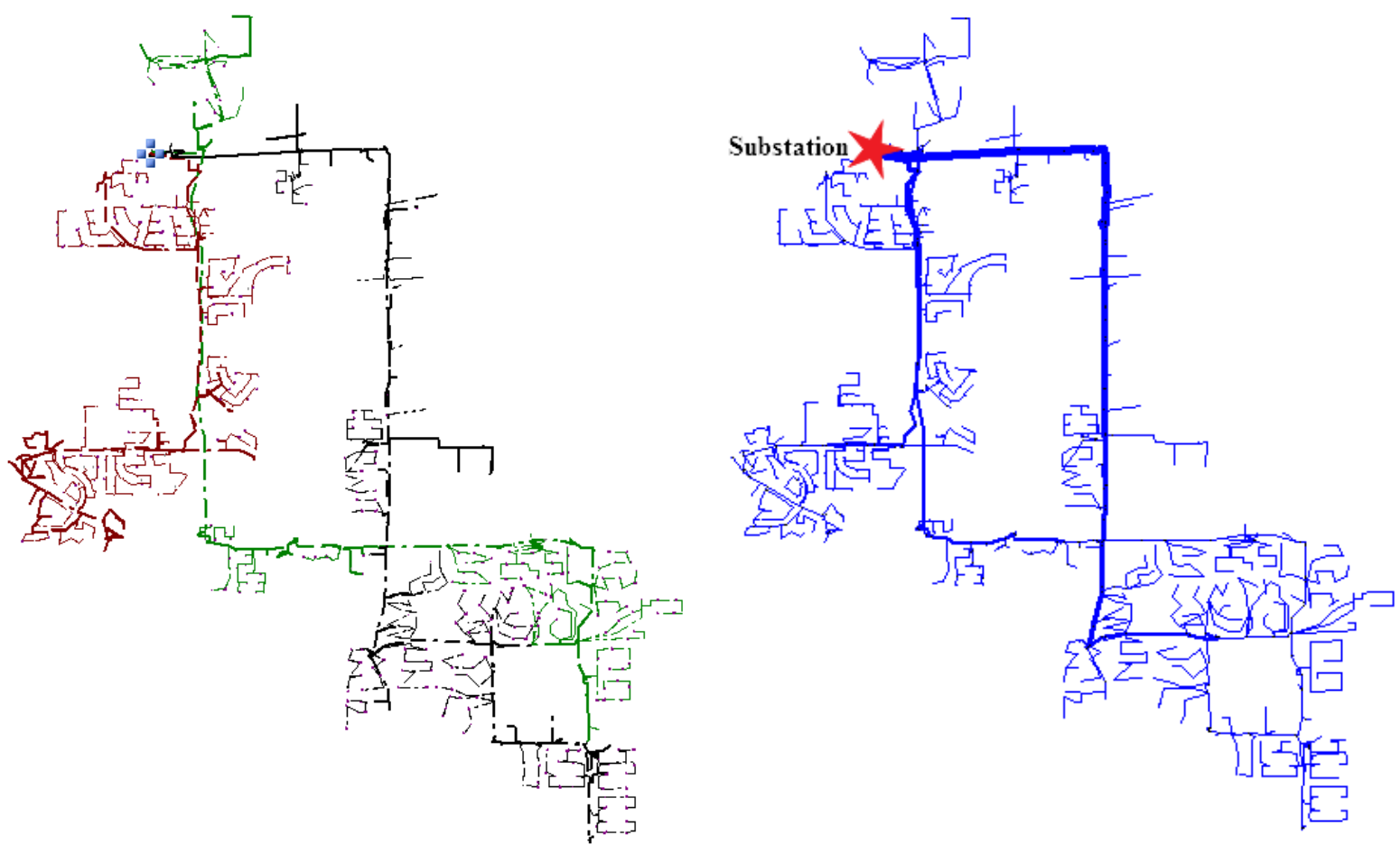

Figure 26. Geographical topology view of the PG\&E distribution system in CYMDIST and OpenDSS

An imporant note is that PG\&E has implemented a traditional CVR program for many years, in which local control settings are such that end of line customer voltages are maintained in the lower portion of the acceptable range under peak loading conditions. More recently, PG\&E has piloted centralized VVO schemes to evaluate potential for improved monitoring and control of circuit voltages and increased savings available from such centralized VVO control. For the purposes of this study, voltage reduction refers to the incremental voltage reduction achieved in addition to the traditional voltage reduction savings PG\&E already benefits from today.

Figure 27 shows the current profile of the distribution system as well as the error (obtained at full load) between the CYMDIST and OpenDSS models as a function of distance. All current errors were less than $2.5 \%$. 

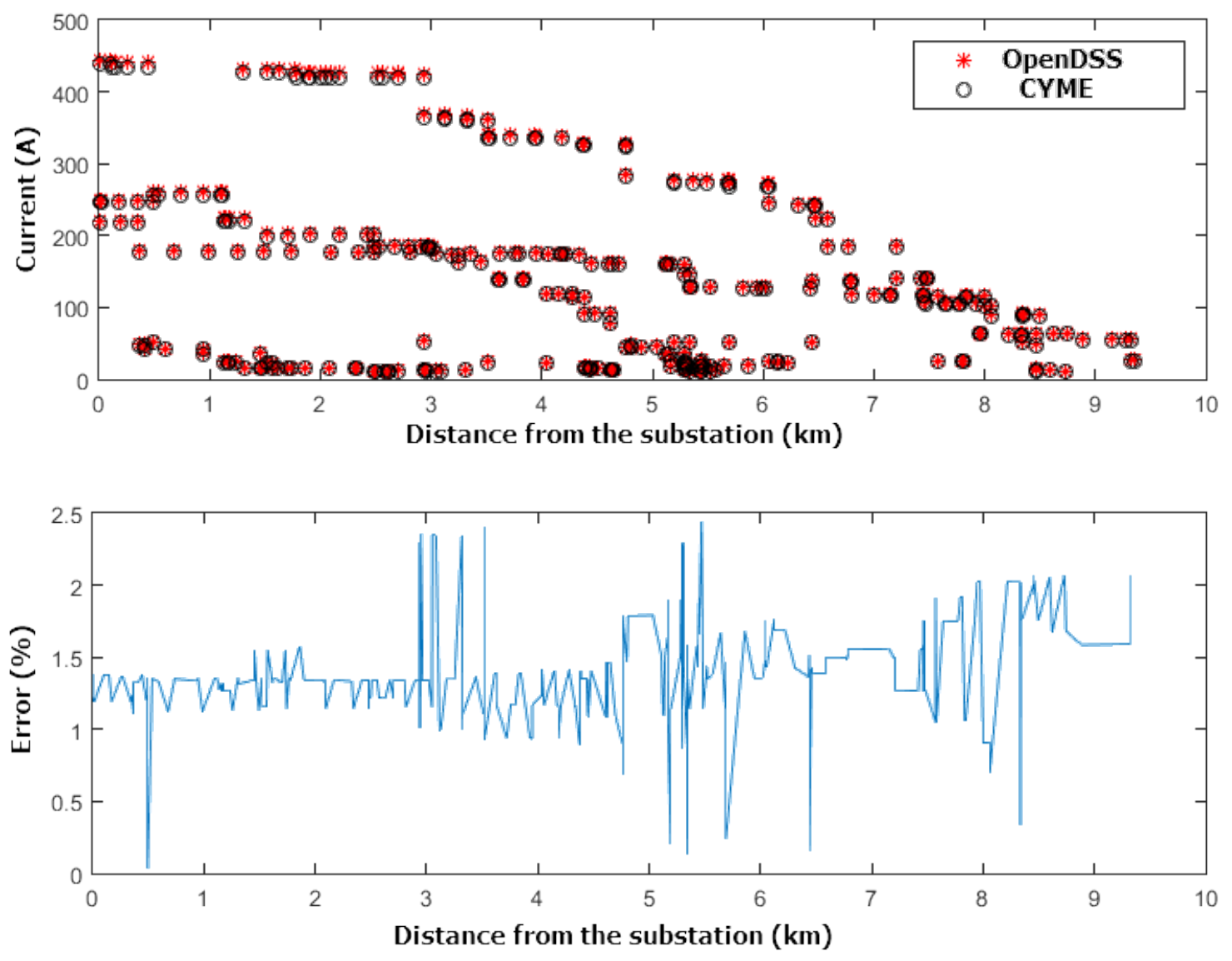

Figure 27. Current profile and current error of the PG\&E distribution system

Figure 28 shows the positive sequence impedance profile of the distribution system. All impedance errors were less than $3.15 \%$. Similar to the HECO distribution system, impedance errors increased toward the end of the lines, as is typical.

Based on Figure 26 through Figure 28, it was concluded that the model was accurately converted to OpenDSS. 

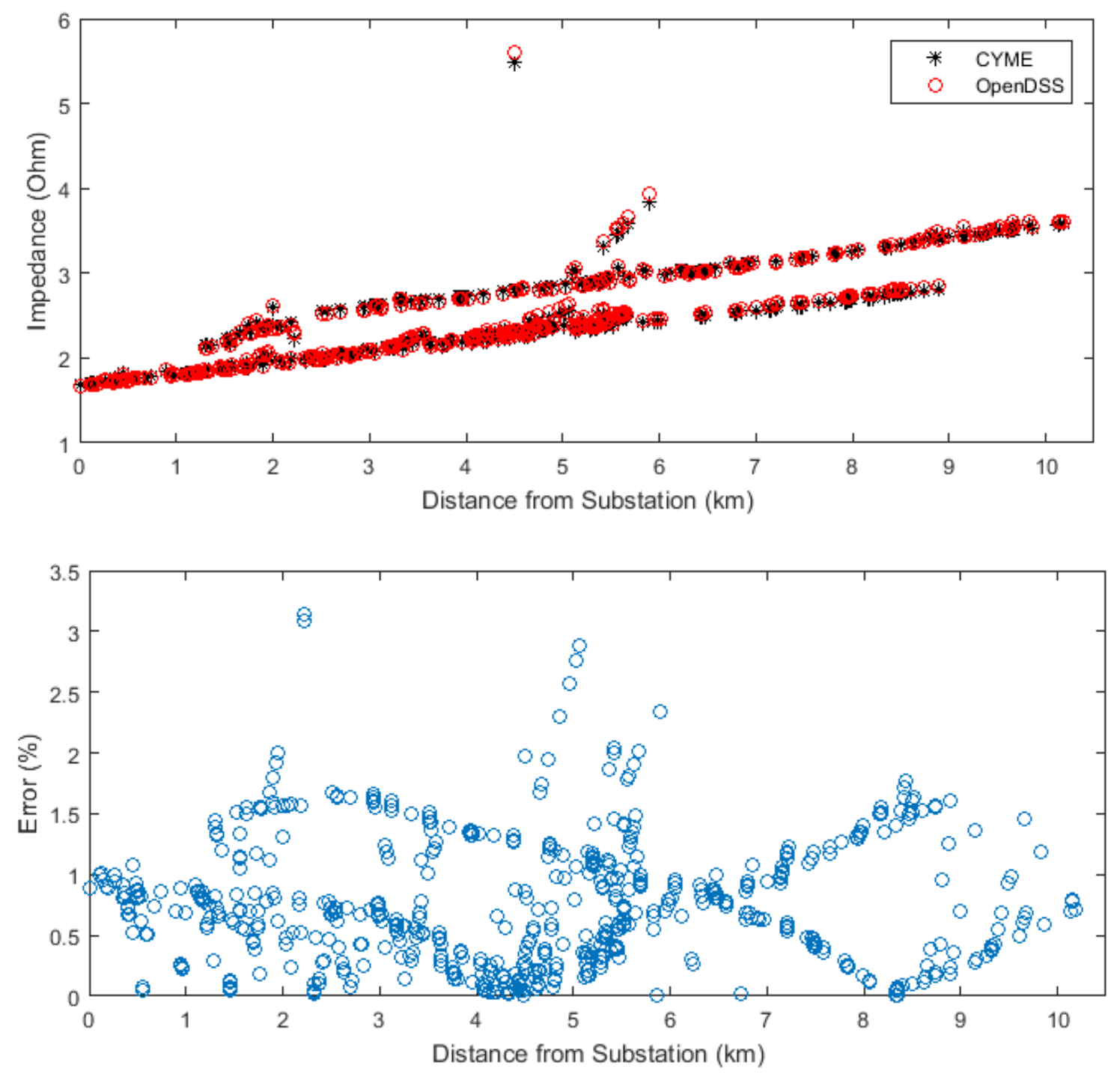

Figure 28. Positive sequence impedance profile and sequence impedance error of the PG\&E distribution system

The model provided by PG\&E did not include any secondary, low voltage circuits. These were necessary for the analysis and were added into the validated OpenDSS model. As there was limited information about a typical secondary model, all customers were placed at the end of a secondary line of 50 feet. Different conductor types were selected based on the size of the transformers and load.

\subsubsection{Annual Photovoltaic and Load Data}

Table 17 summarizes the PV and load data measurements for PG\&E distribution system, taken from PG\&E’s SCADA system and SolarCity's California PV output database. 
Table 17. Annual PV and Load Data Summary for the PG\&E Distribution System

\begin{tabular}{lll}
\hline Measurement Name & Source & Data Resolution \\
\hline Substation Active Power & SCADA Telemetry & $15 \mathrm{~min}-1 \mathrm{hour}$ \\
\hline PV Irradiation & PV/Inverter Output & $15 \mathrm{sec}-60 \mathrm{sec}$ \\
\hline
\end{tabular}

PG\&E provided 15-minute SCADA data for the substation transformer bank as well as the individual circuits. This data set included phase current data for each of the three circuits fed by the transformer bank. Nine single-phase loadshapes were created from the phase current SCADA data. Each of the profiles was normalized based on the maximum value from all nine profiles. In addition, one three-phase loadshape was created per circuit by averaging the three single-phase current values. These loadshapes were assigned to each load based on their respective feeder and phase. For example, if a load was on phase A of circuit A, it would be assigned the loadshape for circuit A phase A. Note that loadshapes in OpenDSS are considered to be three-phase, singlephase currents were multiplied by the square root of three to ensure proper loading. These values were validated in the simulation to ensure the sum of the three circuits load in the simulation closely matched the SCADA load data at the substation bank.

A similar process was performed for the PV profiles using data provided by SolarCity, including inverter output data for California PV systems. The load and PV profiles were applied to the appropriate load and PV objects in order to perform QSTS simulation.

\subsubsection{Simulation Results}

This section presents simulation results for the PG\&E distribution system. Scenarios without PV were first studied to obtain base case results. Following this, multiple scenarios with various PV penetrations and smart inverter densities were studied to evaluate their impact on voltage reduction energy savings and power quality. Finally, multiple scenarios without VO were studied to evaluate PV and smart inverter impact on power quality.

\subsubsection{Base Case}

All PV systems currently existing on the PG\&E distribution system were first removed from the model before two different scenarios were studied - base case and VO without PV. The QSTS simulation was conducted for one year with 8,760 1-hour time steps.

As shown later in Table 21, there were base case voltage reduction energy savings of 3.86\% without PV. Table 18 compares the power quality scores obtained for base case and VO without PV. With VO implemented, the PQS was reduced from 9.2482 to 8.6729 . For both cases, system voltage magnitudes were always within ANSI limits. Therefore, SAVMVI was always 10. Power quality scores are presented in two forms - the raw value and the score after interpolation. A value of 10 represents an ideal case. VO implementation caused the raw value of SAVFI to moderately increase and the raw value of SAVUI to very slightly decrease. This led to a lower individual score for SAVFI and SAVUI. The raw values of SRPDI and SELI decreased leading to higher individual scores. VO implementation caused the LTC to change taps more frequently and increase the raw value of SCDOI, reducing the score of SCDOI by almost half. This contributed the most to the lower overall PQS. 
Table 18. PG\&E Power Quality Scores for Base Case and CVR VO without PV

\begin{tabular}{|c|c|c|c|c|c|c|c|c|c|}
\hline \multicolumn{2}{|c|}{ Scenario } & & SAVMV & SAVFI & SAVUI & SRPDI & SELI & SCDOI & $P Q S$ \\
\hline \multirow{4}{*}{$\begin{array}{l}\text { D } \\
\text { O } \\
Z\end{array}$} & \multirow{2}{*}{$\begin{array}{l}\text { Base } \\
\text { Case }\end{array}$} & $\begin{array}{c}\text { Raw } \\
\text { Value }\end{array}$ & 0 & 0.0014 & 0.0009 & 6.2279 & 0.0123 & 0.0289 & \multirow[t]{2}{*}{9.2482} \\
\hline & & Score & 10 & 9.5296 & 9.8648 & 7.6934 & 8.6325 & 9.7687 & \\
\hline & \multirow{2}{*}{$\begin{array}{c}\text { CVR } \\
\text { VO }\end{array}$} & $\begin{array}{c}\text { Raw } \\
\text { Value }\end{array}$ & 0 & 0.03 & 0.0009 & 2.19 & 0.0112 & 0.3391 & \multirow{2}{*}{8.6729} \\
\hline & & Score & 10 & 8.9847 & 9.8639 & 9.1889 & 8.7575 & 5.2421 & \\
\hline
\end{tabular}

\subsubsection{Voltage Reduction Energy Savings and Power Quality Score with Photovoltaic and Smart Inverters}

Various scenarios of different PV penetrations and smart inverter densities were studied. PV penetration scenarios included $5 \%, 10 \%, 20 \%, 30 \%, 50 \%$ and $100 \%$ and smart inverter densities included $0 \%, 25 \%, 50 \%$ and $100 \%$. The locations of the PV systems as well as the allocation of smart inverters were randomly selected following the approach given in Section 4.3. This random allocation of $\mathrm{PV}$ resulted in a large system being installed when the PV penetration scenario was reaching $25 \%$, preventing $25 \%$ from being studied. For this reason, PV penetration scenarios of $20 \%$ and $30 \%$ were studied rather than $25 \%$. Twenty-four (24) different scenarios of $\mathrm{PV}$ penetration and smart inverter density were studied with VO implemented.

\subsection{Volt-VAR Curve Selection}

The best VVC for voltage reduction was selected using the approach outlined in 4.4. Four weeks, including the first week of January, April, July and October, were studied under 5\% PV penetration and $25 \%$ smart inverter penetration. Table 7 lists all the VVC studied and the best VVC parameters selected. The best VVC to achieve the maximum voltage reduction energy savings is shown in Figure 29.

Table 19. PG\&E Distribution System's VVC Selection

\begin{tabular}{c|c|c}
\hline Assumptions & Scenarios Studied for VVC Selection & Selected \\
\hline $\begin{array}{c}\text { VVC Width }=0.01 \mathrm{pu} \\
\text { Dead Band Width }=0.002 \mathrm{pu}\end{array}$ & VVC Center $=0.955,0.96,0.965,0.97,0.98 \mathrm{pu}$ & $0.96 \mathrm{pu}$ \\
\hline $\begin{array}{c}\text { VVC Center }=0.96 \mathrm{pu} \\
\text { Dead Band Width }=0.002 \mathrm{pu}\end{array}$ & VVC Width $=0.01,0.02,0.03,0.05,0.08 \mathrm{pu}$ & $0.01 \mathrm{pu}$ \\
\hline VVC Center $=0.96 \mathrm{pu}$ & $\begin{array}{c}\text { Dead Band Width }=0.001,0.002,0.003,0.005 \\
0.008,0.1 \mathrm{pu}\end{array}$ & $0.001 \mathrm{pu}$ \\
VVC Width $=0.01 \mathrm{pu}$ & \multicolumn{2}{c}{}
\end{tabular}




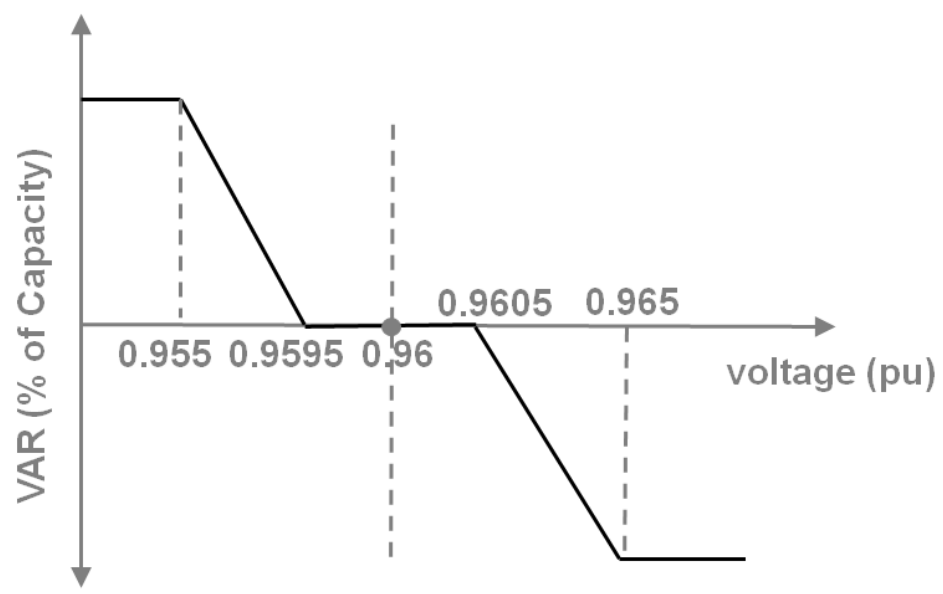

Figure 29. VVC selected to achieve the max voltage reduction energy savings on the PG\&E distribution system.

(In this study, the reactive power capability was limited to $60 \%$ of kVA nameplate.)

\subsection{Voltage Profile}

Table 8 shows the voltage data summary for all 24 scenarios, including the maximum voltage, minimum voltage, and average voltage of the PG\&E distribution system. The maximum and minimum voltages were always around 0.97 to $0.979 \mathrm{pu}$ and 0.95 to $0.9557 \mathrm{pu}$ respectively. When PV penetration was 5\%, the greatest voltage reduction effect occurred when smart inverter density was $25 \%$. When PV penetration was $50 \%$, the greatest voltage reduction effect occurs when smart inverter density was $50 \%$. When PV penetration was $10 \%, 20 \%, 30 \%$ or $100 \%$, the greatest voltage reduction effect occurred when smart inverter density was $100 \%$.

Under the same PV penetration, a higher smart inverter penetration helped reduce the voltage profile in most scenarios. With the same amount of smart inverters on the system, a higher PV penetration did not necessarily cause voltage to increase. Instead, the higher PV penetration caused the average voltage to decrease in most scenarios. Under a higher PV penetration, but the same smart inverter penetration, the total amount of smart inverters became greater, so the voltage reduction effect was more significant after implementing VO. 
Table 20. Voltage Results by PV Penetration and Smart Inverter Density for the PG\&E Distribution System

\begin{tabular}{c|c|ccc}
\hline \multicolumn{2}{c|}{ Scenario } & \multicolumn{3}{c}{ Voltage Result } \\
\hline $\boldsymbol{P V}$ & $\begin{array}{c}\text { Smart } \\
\text { Inverter }\end{array}$ & Maximum & Minimum & Average \\
\hline \multirow{5}{*}{$5 \%$} & 0 & 0.9787 & 0.9557 & 0.9723 \\
& $25 \%$ & 0.9772 & 0.9542 & 0.9709 \\
& $50 \%$ & 0.9774 & 0.9544 & 0.9711 \\
& $100 \%$ & 0.9757 & 0.9543 & 0.9709 \\
\hline \multirow{5}{*}{$10 \%$} & 0 & 0.9784 & 0.9555 & 0.9716 \\
& $25 \%$ & 0.9773 & 0.9544 & 0.9706 \\
& $50 \%$ & 0.9769 & 0.9544 & 0.9704 \\
$20 \%$ & $100 \%$ & 0.9753 & 0.9542 & 0.9700 \\
\hline \multirow{5}{*}{$5 \%$} & 0 & 0.9790 & 0.9557 & 0.9721 \\
& $25 \%$ & 0.9774 & 0.9545 & 0.9707 \\
& $50 \%$ & 0.9772 & 0.9544 & 0.9704 \\
& $100 \%$ & 0.9753 & 0.9542 & 0.9700 \\
\hline \multirow{5}{*}{$50 \%$} & 0 & 0.9788 & 0.9557 & 0.9720 \\
& $25 \%$ & 0.9772 & 0.9544 & 0.9705 \\
& $50 \%$ & 0.9767 & 0.9542 & 0.9700 \\
& $100 \%$ & 0.9752 & 0.9533 & 0.9699 \\
\hline \multirow{5}{*}{$50 \%$} & 0 & 0.9783 & 0.9555 & 0.9717 \\
& $25 \%$ & 0.9763 & 0.9540 & 0.9698 \\
& $50 \%$ & 0.9760 & 0.9532 & 0.9696 \\
& $100 \%$ & 0.9751 & 0.9535 & 0.9697 \\
\hline \multirow{5}{*}{$50 \%$} & 0 & 0.9767 & 0.9554 & 0.9710 \\
& $25 \%$ & 0.9747 & 0.9531 & 0.9688 \\
& $50 \%$ & 0.9749 & 0.9532 & 0.9687 \\
\hline
\end{tabular}

\subsection{Voltage Reduction Benefit}

Table 21 shows voltage reduction energy savings for all $24 \mathrm{PV}$ scenarios and the base case $(0 \%$ $\mathrm{PV}$ and $0 \% \mathrm{SI}$ ). With only one exception, a higher smart inverter density with PV penetration remaining constant led to larger energy savings, with the highest voltage reduction savings at $100 \%$ smart inverter penetration (Figure 30). With a few exceptions, a higher PV penetration similarly led to larger voltage reduction energy savings (Figure 31). 
Table 21. Voltage Reduction Energy Savings by PV Penetration and Smart Inverter Density for the PG\&E Distribution System ${ }^{2}$

\begin{tabular}{c|c|c|c|c|c}
\hline \multirow{2}{*}{$\begin{array}{l}\text { Voltage Reduction } \\
\text { Energy Savings (\%) }\end{array}$} & \multicolumn{5}{|c}{ Smart Inverter Density } \\
\cline { 2 - 6 } & $\mathbf{0 \%}$ & $\mathbf{2 5 \%}$ & $\mathbf{5 0} \%$ & $\mathbf{1 0 0 \%}$ \\
\hline \multirow{4}{*}{ PV Penetration } & $\mathbf{0 \%}$ & 3.86 & & & \\
\cline { 3 - 6 } & $\mathbf{5 \%}$ & 3.84 & 3.96 & 3.94 & 3.97 \\
\cline { 2 - 6 } & $\mathbf{1 0 \%}$ & 3.88 & 3.97 & 3.99 & 4.02 \\
\cline { 3 - 6 } & $\mathbf{2 0 \%}$ & 3.83 & 3.95 & 3.98 & 4.06 \\
\cline { 3 - 6 } & $\mathbf{3 0} \%$ & 3.84 & 3.97 & 4.03 & 4.08 \\
\hline & $\mathbf{5 0 \%}$ & 3.85 & 4.02 & 4.08 & 4.09 \\
\hline & $\mathbf{1 0 0 \%}$ & 3.89 & 4.15 & 4.18 & 4.30 \\
\hline
\end{tabular}

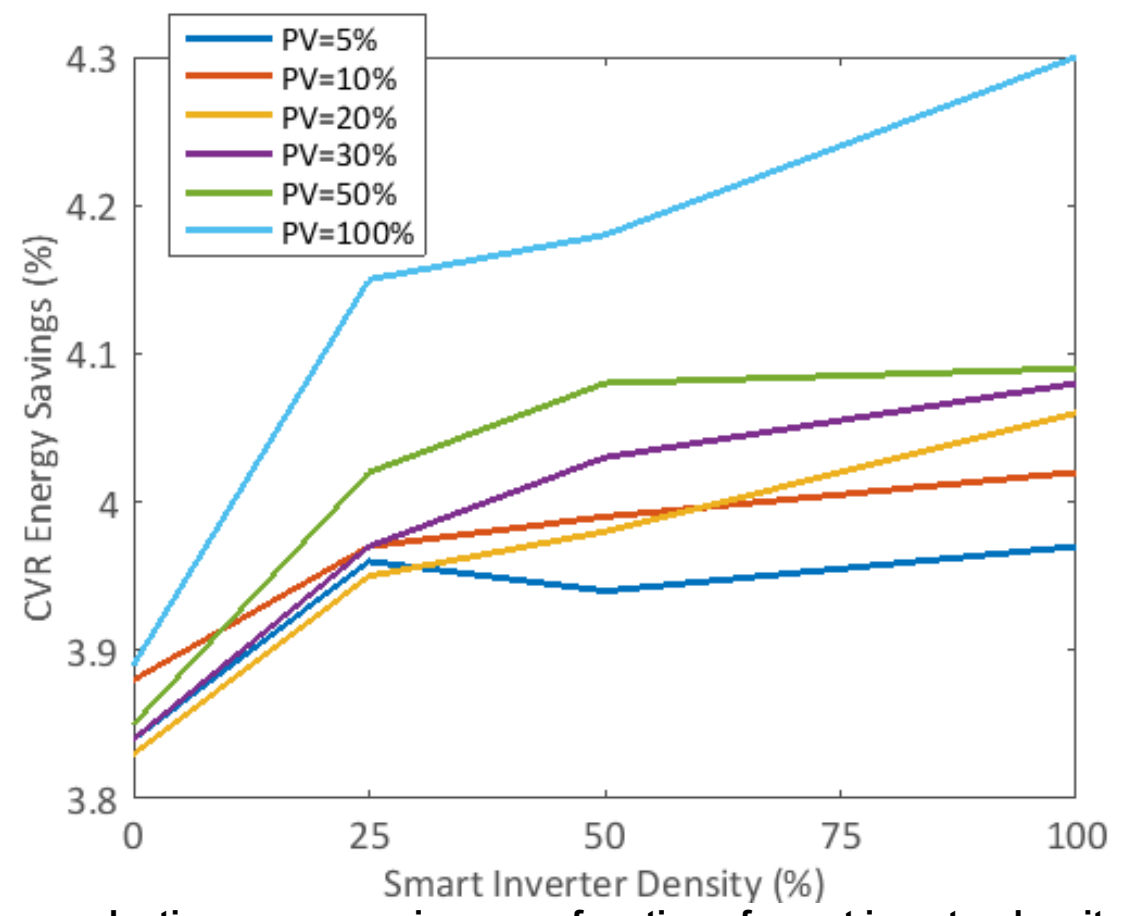

Figure 30. Voltage reduction energy savings as a function of smart inverter density at different PV penetrations for the PG\&E distribution system

\footnotetext{
${ }^{2}$ These savings were based on modeling the loads with a CVR factor of 0.8 . If the CVR factor for the load type studied is known to be higher or lower, an approximately linear increase or decrease in savings is expected.
} 


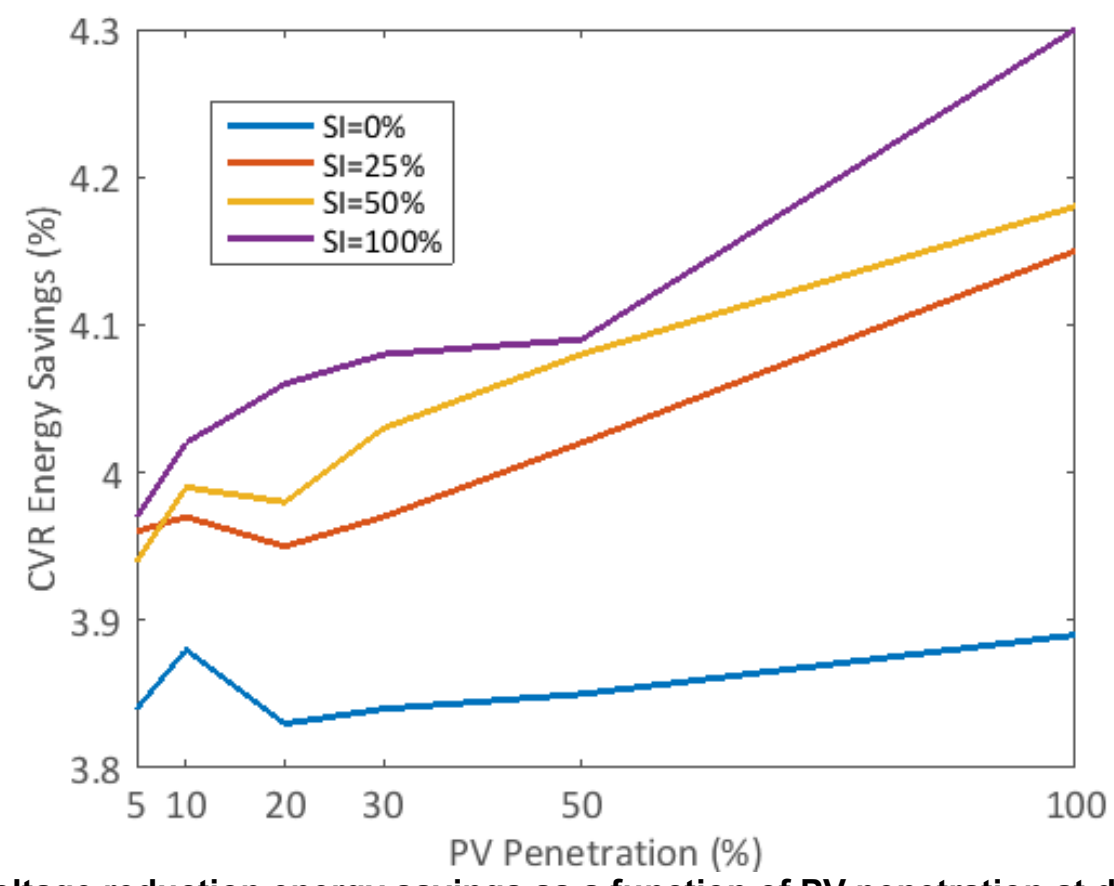

Figure 31. Voltage reduction energy savings as a function of PV penetration at different smart inverter densities for the PG\&E distribution system

\subsection{Power Quality Score}

Table 22 and Table 23 show the results of the power quality indices for all 24 scenarios, with the raw values and interpolated scores respectively. Table 24 shows the combined PQS. Compared with the result in Table 18, the combination of CVR VO being active and smart inverter voltVAR control reduced the score for SCDOI and SRPDI in order to increase energy savings at the load nodes. This indicates more regulator tap changes and increased reactive power demand. The increased reactive power demand occurred because the volt-VAR control of the smart inverter absorbs VARs to lower voltage, leading to a higher metric value of SRPDI. With this voltage reduction, more regulator tap changes have to occur to lower the voltage. These lower scores were a result of this iteration of the VO implementation and different results would most likely occur with a more sophisticated voltage reduction control approach that optimizes for reactive power demand and CVR (commonly referred to as VVO). Further, with VO being active, it was difficult to differentiate PV penetration's and smart inverter density's impact vs. the impact of voltage reduction on the power quality score. 
Table 22. Power Quality Score Raw Values by PV Penetration and Smart Inverter Density for the PG\&E Distribution System

\begin{tabular}{c|c|cccccc}
\hline \multicolumn{2}{c|}{ Scenario } & \multicolumn{6}{c}{ Power Quality Metric Value } \\
\hline $\boldsymbol{P}$ S & $\begin{array}{c}\text { Smart } \\
\text { Inverter }\end{array}$ & SAVMVI & SAVFI & SAVUI & SRPDI & SELI & SCDOI \\
\hline \multirow{5}{*}{$5 \%$} & 0 & 0 & 0.003187 & 0.000899 & 2.355898 & 0.011054 & 0.369626 \\
& $25 \%$ & 0 & 0.003094 & 0.000867 & 2.433243 & 0.011116 & 0.379836 \\
& $50 \%$ & 0 & 0.003045 & 0.000877 & 2.499562 & 0.011147 & 0.372700 \\
& $100 \%$ & 0 & 0.003012 & 0.000853 & 2.507397 & 0.011220 & 0.371395 \\
\hline \multirow{5}{*}{$10 \%$} & 0 & 0 & 0.003228 & 0.000883 & 2.321062 & 0.010917 & 0.375758 \\
& $25 \%$ & 0 & 0.003130 & 0.000859 & 2.616518 & 0.011015 & 0.387358 \\
& $50 \%$ & 0 & 0.003017 & 0.000832 & 2.699033 & 0.011104 & 0.383129 \\
& $100 \%$ & 0 & 0.003140 & 0.000814 & 3.041552 & 0.011280 & 0.416530 \\
\hline \multirow{5}{*}{$20 \%$} & 0 & 0 & 0.003306 & 0.000971 & 2.165262 & 0.010408 & 0.369203 \\
& $25 \%$ & 0 & 0.003135 & 0.000920 & 2.500720 & 0.010603 & 0.385538 \\
& $50 \%$ & 0 & 0.003233 & 0.000895 & 2.884725 & 0.010783 & 0.422101 \\
& $100 \%$ & 0 & 0.003097 & 0.000769 & 2.982243 & 0.010970 & 0.433139 \\
\hline \multirow{5}{*}{$30 \%$} & 0 & 0 & 0.003267 & 0.000956 & 2.169961 & 0.010161 & 0.368812 \\
& $25 \%$ & 0 & 0.003122 & 0.000882 & 2.531137 & 0.010381 & 0.395571 \\
& $50 \%$ & 0 & 0.003144 & 0.000786 & 2.879758 & 0.010649 & 0.431185 \\
& $100 \%$ & 0 & 0.002936 & 0.000707 & 3.318986 & 0.010638 & 0.433578 \\
\hline \multirow{5}{*}{$50 \%$} & 0 & 0 & 0.003336 & 0.000859 & 2.144614 & 0.009609 & 0.386896 \\
& $25 \%$ & 0 & 0.003070 & 0.000806 & 2.903859 & 0.010081 & 0.430450 \\
& $50 \%$ & 0 & 0.002926 & 0.000655 & 3.313129 & 0.010144 & 0.447462 \\
& $100 \%$ & 0 & 0.003094 & 0.000637 & 3.853966 & 0.010425 & 0.462477 \\
\hline \multirow{5}{*}{$100 \%$} & 0 & 0 & 0.003152 & 0.000818 & 2.427623 & 0.009863 & 0.384538 \\
& $25 \%$ & 0 & 0.002886 & 0.000627 & 3.312363 & 0.010456 & 0.467544 \\
& $50 \%$ & 0 & 0.003010 & 0.000619 & 3.872574 & 0.010750 & 0.481332 \\
& $100 \%$ & 0 & 0.003204 & 0.000632 & 4.470404 & 0.011101 & 0.484133 \\
\hline
\end{tabular}


Table 23. Power Quality Score by PV Penetration and Smart Inverter Density for the PG\&E Distribution System

\begin{tabular}{c|c|ccccccc}
\hline \multicolumn{8}{c|}{ Scenario } & \multicolumn{7}{c}{ Individual Score for Each Power Quality Metric } \\
\hline \multirow{2}{*}{$\boldsymbol{P V}$} & SI & SAVMVI & SAVFI & SAVUI & SRPDI & SELI & SCDOI & PQS \\
\hline \multirow{5}{*}{$5 \%$} & 0 & 10 & 8.93770 & 9.865131 & 9.127445 & 8.771792 & 4.774612 & 8.579448 \\
& $25 \%$ & 10 & 8.96860 & 9.869876 & 9.098799 & 8.764933 & 4.261214 & 8.493905 \\
& $50 \%$ & 10 & 8.98486 & 9.868471 & 9.074236 & 8.761498 & 4.533881 & 8.537158 \\
& $100 \%$ & 10 & 8.99615 & 9.872054 & 9.071334 & 8.753319 & 4.620638 & 8.552249 \\
\hline \multirow{5}{*}{$10 \%$} & 0 & 10 & 8.92406 & 9.867559 & 9.140348 & 8.786995 & 4.894330 & 8.602217 \\
& $25 \%$ & 10 & 8.95669 & 9.871084 & 9.030919 & 8.776115 & 4.615800 & 8.541769 \\
& $50 \%$ & 10 & 8.99421 & 9.875193 & 9.000358 & 8.766254 & 4.746100 & 8.563686 \\
& $100 \%$ & 10 & 8.95338 & 9.877901 & 8.873499 & 8.746618 & 4.684940 & 8.522723 \\
\hline \multirow{5}{*}{$20 \%$} & 0 & 10 & 8.89816 & 9.854283 & 9.198051 & 8.843520 & 4.957999 & 8.625336 \\
& $25 \%$ & 10 & 8.95509 & 9.861985 & 9.073807 & 8.821913 & 4.581602 & 8.549068 \\
& $50 \%$ & 10 & 8.92237 & 9.865741 & 8.931583 & 8.801895 & 4.576629 & 8.516371 \\
& $100 \%$ & 10 & 8.96764 & 9.884720 & 8.895466 & 8.781145 & 4.446355 & 8.495888 \\
\hline \multirow{5}{*}{$30 \%$} & 0 & 10 & 8.91086 & 9.856541 & 9.196311 & 8.871009 & 4.936592 & 8.628554 \\
& $25 \%$ & 10 & 8.95949 & 9.867678 & 9.062542 & 8.846588 & 4.517642 & 8.542324 \\
& $50 \%$ & 10 & 8.95208 & 9.882143 & 8.933423 & 8.816825 & 4.419546 & 8.500670 \\
& $100 \%$ & 10 & 9.02142 & 9.893942 & 8.770746 & 8.818044 & 4.273169 & 8.462887 \\
\hline \multirow{5}{*}{$50 \%$} & 0 & 10 & 8.88791 & 9.871162 & 9.205699 & 8.932372 & 4.616096 & 8.585540 \\
& $25 \%$ & 10 & 8.97673 & 9.879119 & 8.924497 & 8.879936 & 4.211338 & 8.478604 \\
& $50 \%$ & 10 & 9.02461 & 9.901700 & 8.772915 & 8.872940 & 3.944095 & 8.419378 \\
& $100 \%$ & 10 & 8.96858 & 9.904431 & 8.572605 & 8.841658 & 3.797983 & 8.347544 \\
\hline \multirow{5}{*}{$100 \%$} & 0 & 10 & 8.94947 & 9.877371 & 9.100880 & 8.904071 & 4.356616 & 8.531401 \\
& $25 \%$ & 10 & 9.03808 & 9.905996 & 8.773199 & 8.838230 & 3.361451 & 8.319493 \\
& $50 \%$ & 10 & 8.99677 & 9.907116 & 8.565713 & 8.805588 & 3.184977 & 8.243362 \\
& $100 \%$ & 10 & 8.93193 & 9.905145 & 8.344295 & 8.766571 & 3.310989 & 8.209822 \\
\hline \multirow{5}{*}{} & & & & & & & &
\end{tabular}

Table 24. Power Quality Score Summary for the PG\&E Distribution System

\begin{tabular}{|c|c|c|c|c|c|}
\hline \multirow{2}{*}{\multicolumn{2}{|c|}{ PQS }} & \multicolumn{4}{|c|}{ Smart Inverter Density } \\
\hline & & $0 \%$ & $25 \%$ & $50 \%$ & $100 \%$ \\
\hline \multirow{7}{*}{$\begin{array}{c}\text { PV } \\
\text { Penetration }\end{array}$} & $0 \%$ & 8.67 & & & \\
\hline & $5 \%$ & 8.58 & 8.49 & 8.54 & 8.55 \\
\hline & $10 \%$ & 8.6 & 8.54 & 8.56 & 8.52 \\
\hline & $20 \%$ & 8.63 & 8.55 & 8.52 & 8.5 \\
\hline & $30 \%$ & 8.63 & 8.54 & 8.5 & 8.46 \\
\hline & $50 \%$ & 8.59 & 8.48 & 8.42 & 8.35 \\
\hline & $100 \%$ & 8.53 & 8.32 & 8.24 & 8.21 \\
\hline
\end{tabular}

\subsubsection{Power Quality Score without CVR VO}

The previous simulation results showed that VO can help save energy, but may come at the expense of power quality, depending on the VO implementation. In order to assess PV penetration's and smart inverter density's impact on PQS, absent VO, more simulation scenarios were studied. 
The purpose of this additional study was to investigate how smart inverters can improve distribution system power quality. The previous VVC was selected to achieve maximum voltage reduction energy savings and was based on a lower system voltage. In this study, various VVC centers, including $0.96,0.97,0.98,0.99,1.0,1.01$ and $1.02 \mathrm{pu}$, were analyzed to find the curve that achieved the highest power quality score. The results are shown in Figure 32. A PV penetration and smart inverter density of 50\% was used. Compared with base case, no PV score of 9.32, all non-VO scenarios with PV and smart inverters had higher power quality scores, with a band center of $1.0 \mathrm{pu}$ yielding the highest power quality. This new curve center of $1.0 \mathrm{pu}$ was used for all new scenarios.

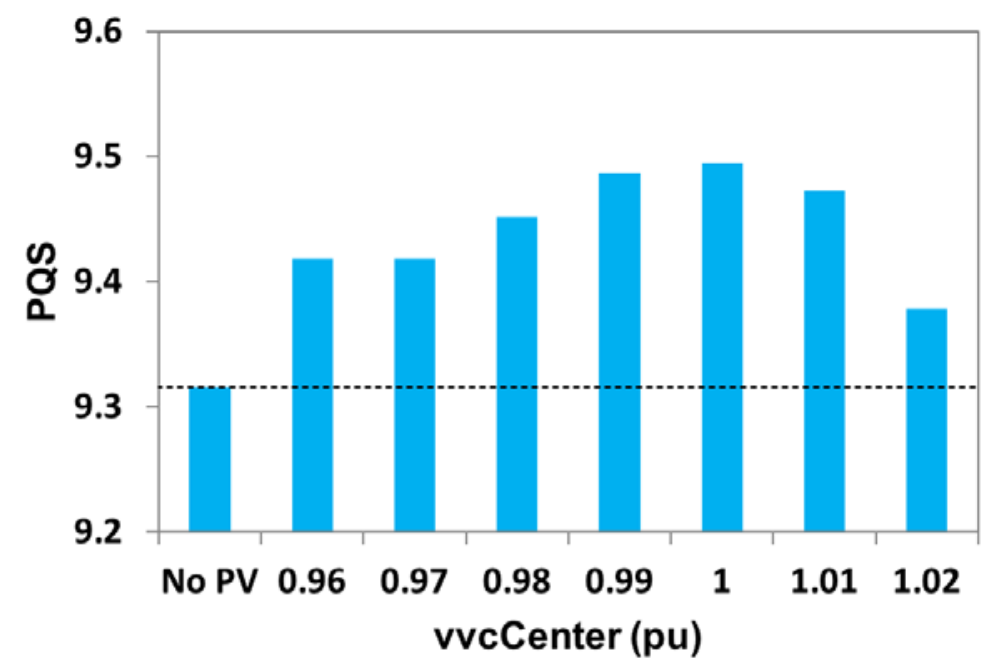

Figure 32. PQS results with different VVC centers for the PG\&E distribution system

Table 25 and Table 26 show the results of the power quality indices for all 24 non-VO scenarios, with the raw values and interpolated scores respectively. Table 27 shows the combined PQS. The combined PQS improves for all scenarios with smart inverters except for the $100 \% \mathrm{PV}$ penetration and $100 \%$ smart inverter density. A higher PV penetration typically resulted in an increase in voltage fluctuation and voltage regulator tap changes when compared to the base case in Table 18. As a result, the individual scores of SAVFI and SCDOI decreased. However, reactive power demand and system losses typically improved with increasing PV penetration and the individual scores of SRPDI and SELI increased. SAVUI did not have a consistent pattern and SAVMVI remained at a perfect 10 across all scenarios, signifying no steady-state voltage issues. There were minimal capacitor switching operations observed in these simulations because the system voltages stayed within the acceptable range of the capacitor settings. Therefore, the $\mathrm{SCDOI}_{\text {Cap }}$ was close to 10 for all scenarios without VO.

The decrease in the metric for SCDOI could have possibly improved under different LTC settings. The settings were not made for all of the PV penetration and smart inverter scenarios studied. The time left in the project did not allow running a sensitivity analysis on the LTC settings, but there was potential to demonstrate a power quality improvement over base case across al PV penetration scenarios studied. 
Table 25. Power Quality Score Raw Values without CVR VO for the PG\&E Distribution System

\begin{tabular}{c|c|cccccc}
\hline \multicolumn{2}{c|}{ Scenario } & \multicolumn{7}{c}{ Power Quality Metric Value } \\
\hline $\boldsymbol{P V}$ & $\begin{array}{c}\text { Smart } \\
\text { Inverter }\end{array}$ & SAVMVI & SAVFI & SAVUI & SRPDI & SELI & SCDOI \\
\hline \multirow{5}{*}{$5 \%$} & 0 & 0 & 0.001446 & 0.000892 & 6.233543 & 0.012022 & 0.030625 \\
& $25 \%$ & 0 & 0.001432 & 0.000869 & 6.045961 & 0.012106 & 0.030625 \\
& $50 \%$ & 0 & 0.001428 & 0.000912 & 5.868666 & 0.012130 & 0.030625 \\
& $100 \%$ & 0 & 0.001409 & 0.000906 & 5.499959 & 0.012226 & 0.030738 \\
\hline \multirow{5}{*}{$10 \%$} & 0 & 0 & 0.001475 & 0.000877 & 6.232042 & 0.011850 & 0.033637 \\
& $25 \%$ & 0 & 0.001456 & 0.000898 & 5.867772 & 0.011952 & 0.033637 \\
& $50 \%$ & 0 & 0.001436 & 0.000890 & 5.497067 & 0.012048 & 0.033637 \\
& $100 \%$ & 0 & 0.001401 & 0.000942 & 4.909338 & 0.012139 & 0.035600 \\
\hline \multirow{5}{*}{$20 \%$} & 0 & 0 & 0.001545 & 0.000957 & 6.246197 & 0.011403 & 0.041571 \\
& $25 \%$ & 0 & 0.001507 & 0.000972 & 5.508814 & 0.011602 & 0.041571 \\
& $50 \%$ & 0 & 0.001471 & 0.001025 & 4.924446 & 0.011688 & 0.043721 \\
& $100 \%$ & 0 & 0.001383 & 0.001049 & 3.521427 & 0.012010 & 0.043657 \\
\hline \multirow{5}{*}{$30 \%$} & 0 & 0 & 0.001601 & 0.000944 & 6.250316 & 0.011191 & 0.046568 \\
& $25 \%$ & 0 & 0.001539 & 0.000963 & 5.194936 & 0.011410 & 0.046568 \\
& $50 \%$ & 0 & 0.001484 & 0.001003 & 4.202135 & 0.011591 & 0.048643 \\
& $100 \%$ & 0 & 0.001398 & 0.000891 & 2.246538 & 0.011683 & 0.054517 \\
\hline \multirow{5}{*}{$50 \%$} & 0 & 0 & 0.001739 & 0.000853 & 6.260133 & 0.010709 & 0.069018 \\
& $25 \%$ & 0 & 0.001643 & 0.001039 & 4.598379 & 0.011010 & 0.071168 \\
& $50 \%$ & 0 & 0.001537 & 0.000845 & 2.269741 & 0.011175 & 0.076825 \\
& $100 \%$ & 0 & 0.001292 & 0.000851 & 1.305563 & 0.011934 & 0.081842 \\
\hline \multirow{5}{*}{$100 \%$} & 0 & 0 & 0.001955 & 0.000813 & 6.229666 & 0.010835 & 0.116033 \\
& $25 \%$ & 0 & 0.001806 & 0.000811 & 2.268107 & 0.011281 & 0.124206 \\
& $50 \%$ & 0 & 0.001584 & 0.000799 & 1.302787 & 0.012014 & 0.128534 \\
& $100 \%$ & 0 & 0.001405 & 0.000847 & 3.600965 & 0.013343 & 0.141353 \\
\hline
\end{tabular}


Table 26. Power Quality Score without CVR VO for the PG\&E Distribution System

\begin{tabular}{c|c|ccccccc}
\hline \multicolumn{7}{c}{ Scenario } & \multicolumn{7}{|c}{ Individual Score for Each Power Quality Metric } \\
\hline \multirow{2}{*}{ PV } & $\begin{array}{c}\text { Smart } \\
\text { Inverte } \\
\end{array}$ & r SAVMVI & SAVFI & SAVUI & SRPDI & SELI & SCDOI & PQS \\
\hline \multirow{5}{*}{$5 \%$} & 0 & 10 & 9.518010 & 9.866181 & 7.691281 & 8.664266 & 9.754999 & 9.249123 \\
& $25 \%$ & 10 & 9.522562 & 9.869613 & 7.760755 & 8.654936 & 9.754999 & 9.260478 \\
& $50 \%$ & 10 & 9.524125 & 9.863171 & 7.826420 & 8.652188 & 9.754999 & 9.270151 \\
& $100 \%$ & 10 & 9.530397 & 9.864072 & 7.962978 & 8.641566 & 9.754100 & 9.292186 \\
\hline \multirow{5}{*}{$10 \%$} & 0 & 10 & 9.508493 & 9.868394 & 7.691836 & 8.683291 & 9.730906 & 9.247153 \\
& $25 \%$ & 10 & 9.514719 & 9.865331 & 7.826751 & 8.671949 & 9.730906 & 9.268276 \\
& $50 \%$ & 10 & 9.521216 & 9.866470 & 7.964049 & 8.661367 & 9.730906 & 9.290668 \\
& $100 \%$ & 10 & 9.533068 & 9.858652 & 8.181727 & 8.651238 & 9.715204 & 9.323315 \\
\hline \multirow{5}{*}{$20 \%$} & 0 & 10 & 9.484979 & 9.856470 & 7.686594 & 8.733024 & 9.667434 & 9.238084 \\
& $25 \%$ & 10 & 9.497712 & 9.854239 & 7.959698 & 8.710888 & 9.667434 & 9.281662 \\
& $50 \%$ & 10 & 9.509701 & 9.846221 & 8.176131 & 8.701348 & 9.650234 & 9.313939 \\
& $100 \%$ & 10 & 9.538922 & 9.842607 & 8.695768 & 8.665503 & 9.650741 & 9.398924 \\
\hline \multirow{5}{*}{$30 \%$} & 0 & 10 & 9.466408 & 9.858346 & 7.685068 & 8.756526 & 9.627459 & 9.232301 \\
& $25 \%$ & 10 & 9.487086 & 9.855479 & 8.075950 & 8.732203 & 9.627459 & 9.296363 \\
& $50 \%$ & 10 & 9.505465 & 9.849548 & 8.443654 & 8.712074 & 9.610859 & 9.353600 \\
& $100 \%$ & 10 & 9.533975 & 9.866342 & 9.167949 & 8.701905 & 9.563864 & 9.472339 \\
\hline \multirow{5}{*}{$50 \%$} & 0 & 10 & 9.420267 & 9.872121 & 7.681432 & 8.810115 & 9.447859 & 9.205299 \\
& $25 \%$ & 10 & 9.452470 & 9.844155 & 8.296897 & 8.776651 & 9.430660 & 9.300139 \\
& $50 \%$ & 10 & 9.487796 & 9.873197 & 9.159355 & 8.758339 & 9.385404 & 9.444015 \\
& $100 \%$ & 10 & 9.569262 & 9.872425 & 9.516458 & 8.673960 & 9.345265 & 9.496228 \\
\hline \multirow{5}{*}{$100 \%$} & 0 & 10 & 9.348167 & 9.878056 & 7.692716 & 8.796074 & 9.071739 & 9.131125 \\
& $25 \%$ & 10 & 9.398028 & 9.878316 & 9.159960 & 8.746538 & 9.006350 & 9.364865 \\
& $50 \%$ & 10 & 9.472064 & 9.880189 & 9.517486 & 8.665063 & 8.971729 & 9.417755 \\
& $100 \%$ & 10 & 9.531609 & 9.872876 & 8.666309 & 8.517394 & 8.869174 & 9.242894 \\
\hline
\end{tabular}

Table 27. Power Quality Score Summary without CVR VO for the PG\&E Distribution System

\begin{tabular}{|c|c|c|c|c|c|}
\hline \multirow{2}{*}{\multicolumn{2}{|c|}{ PQS }} & \multicolumn{4}{|c|}{ Smart Inverter Density } \\
\hline & & $0 \%$ & $25 \%$ & $50 \%$ & $100 \%$ \\
\hline \multirow{7}{*}{$\begin{array}{c}\text { PV } \\
\text { Penetration }\end{array}$} & $0 \%$ & 9.25 & & & \\
\hline & $5 \%$ & 9.25 & 9.26 & 9.27 & 9.29 \\
\hline & $10 \%$ & 9.25 & 9.27 & 9.29 & 9.32 \\
\hline & $20 \%$ & 9.24 & 9.28 & 9.31 & 9.40 \\
\hline & $30 \%$ & 9.23 & 9.30 & 9.35 & 9.47 \\
\hline & $50 \%$ & 9.21 & 9.30 & 9.44 & 9.50 \\
\hline & $100 \%$ & 9.13 & 9.36 & 9.42 & 9.24 \\
\hline
\end{tabular}




\section{Discussions}

This section provides a discussion on a number of important topics and takeaways that are worth noting in this study. This includes the importance of using the same limits for normalizing the power quality metrics; considerations on the impacts of the different characteristics of HECO's and PG\&E's distribution systems on the results; and the amount of PV curtailment observed when prioritizing reactive over active power with smart inverter volt-VAR control. The following sections will discuss each of these topics in detail.

\subsection{Normalizing and Weighting of Power Quality Metrics}

The overall PQS was calculated from six individual power quality scores. Each individual score for SAVMVI, SAVFI, SAVUI, SRPDI, SCDOI and SELI had a value ranging from 0 to 10. These were calculated from the raw scores based on their maximum and minimum limits. For example, to calculate the score for the voltage fluctuation metric SAVFI, the average fluctuation from the entire annual simulation was divided by $3 \%$, a recognized industry limit for fluctuation, and then scaled to a value out of 10 . An average fluctuation of $3 \%$ or greater would result in a score of 0 , whereas a $0 \%$ average voltage fluctuation would result in a score of 10 .

In this study, three different types of limits were used to normalize each of the six metrics to a score of 10, including theoretical limits, industry standard limits, and practical limits. Each of the six individual scores, were given equal weighting when calculating the overall PQS.

1. Theoretical limits. These are theoretical limits set by the physical limits of the system and/or device and are typically the highest. As a result, they will lead to the most optimistic PQS metric. The metric that used theoretical limits in this study was the voltage unbalance index (SAVUI). A theoretical maximum limit of $6.78 \%$ was determined. However, an industry standards limit of 3\% could have been used instead. By dividing by a smaller number, SAVUI would have had a wider range to vary, giving more weight to any increase or decrease in voltage unbalance.

2. Industry standard limits. These limits are based on industry standards and will lead to a more moderate PQS. The indices that used standard limits in this study were the voltage magnitude (SAVMVI), the voltage fluctuation (SAVFI), and the reactive power (SRPDI) indices. For example, SAVFI was based on a 3\% voltage fluctuation. 3\% was chosen based on industry standards for rapid voltage change (RVC). While RVC and voltage fluctuation are different, this standard provided a reasonably proxy to normalize voltage fluctuation.

3. Practical limits. These limits are based on practical references that were found from various sources as well as practical experience. They will lead to the lowest PQS index. The indices that used standard limits in this study were the device operations metric (SCDOI), for voltage regulators and capacitors, and the loss metric (SELI). For example, the practical maximum value for SCDOI for the regulator was 30 operations per day, which was a practical number of possible operations given in [16]. However, the theoretical maximum number of operations for a voltage regulator is 32 operations per hour (768 per day). While this is incredibly unlikely, it indicates the wide range of results that can occur during normalization. On average SCDOI was the metric most impacted by CVR and PV, but it was also normalized with one of the smallest maximum values. 
This suggests that the normalization could also have contributed to the lower score. Another example of a practical maximum is SELI. The maximum value used was $9 \%$, which was based on a small selection of distribution feeders in the EPRI Green Circuits study [8].

To have an ideal PQS from averaged metrics, each metric should be normalized to a score of 10 using the same methodology or standard for maximum and minimum value limits. However, three different types of limits were used in this study due to the limited industry standard information available for defining all six metrics. Thus, due to the different types of limits used in the individual scores, the PQS, despite being an average of six metrics, was not in reality weighted evenly between the six metrics. For example, for the individual score for voltage unbalance, SAVUI, to have a score of zero, the annual average unbalance would have to reach $6.67 \%$. This value of unbalance occurring is practically impossible. In reality, motors on the distribution system would self-protect and trip offline or have detrimental impacts. ANSI C84.12011 for example recommends that voltage unbalance "should be limit to 3\%" [6]. By contrast, the device operations individual score was based on a practical limit. For regulator SCDOI to have a score of zero, each device would need to tap 30 times per day. While this may seem like a lot of operations depending on the utility, it certainly is more likely to occur than a $6.67 \%$ voltage unbalance. As a result, SAVUI would likely have scores between 5.5 (when system average unbalance is $3 \%$ ) and 10 based on the theoretical limits, but SCDOI would likely have scores between 0 and 10 based on the practical limits. This was seen in the results of this study for both the HECO and PG\&E results, but identified too late in the study process to correct. Therefore, while the six individual scores were averaged, in reality the maximum limits used for normalizing the individual scores unintentionally added additional weighting in the calculation of the overall PQS. Specifically, device operation (including capacitor and regulator) and loss indices were more-heavily weighted due to the use of practical limits, skewing the general PQS. Utilizing a consistent type of limit or weighting the limits differently is of interest for future studies.

\subsection{Distribution Systems Comparison: Hawaiian Electric Companies and Pacific Gas and Electric Company}

Although the trends of the results were generally consistent between HECO's and PG\&E's distribution systems, there were differences in the magnitudes of the results. The differences likely are a result of the different characteristics of the two distribution systems. When implementing the VO scheme without PV or smart inverters, the energy savings of PG\&E was $3.86 \%$ while for HECO they were $1.51 \%$. It was theorized that the difference in scores can be partially attributed to:

- Number of capacitors. While the PG\&E distribution system had 7 capacitors, the HECO distribution system had none. The presence of the capacitors likely had a large impact on two evaluation criteria: energy savings and the reactive power score, SRPDI.

The extra capacitors on PG\&E feeder helped achieve higher energy savings, as they were used to flatten the voltage profile. With a flatter voltage profile, the substation LTC could tap lower, thus more energy savings was obtained. On the HECO distribution system, where no capacitor exists, the voltage profile naturally decreased when moving away 
from the substation. This limited the amount that the LTC could tap down, but also provided a greater opportunity for smart inverter volt-VAR control.

In this iteration of the $\mathrm{VO}$ scheme, smart inverters absorb reactive power to further lower the voltage. When capacitors existed, they could help provide a significant amount of this reactive power instead of the substation. When no capacitors were present that reactive power had to be supplied by the substation. Therefore, the SRPDI was lower for the HECO VO simulations when compared to the PG\&E VO simulations.

- Random PV and smart inverter placement. As seen in the HECO Case Study section, there were a few nodes that had a lower voltage, likely caused by the presence of larger loads.

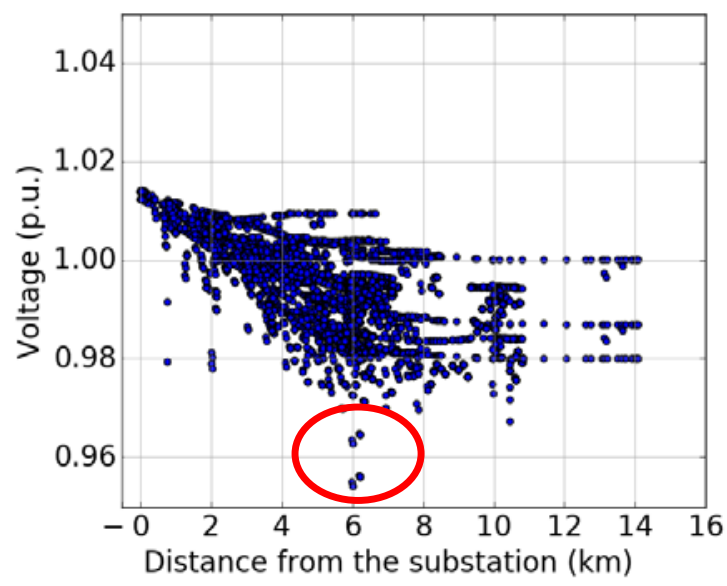

Figure 33. Low-voltage nodes on the HECO distribution system

These low voltage nodes limited the number of taps that the substation LTC could be lowered and thus limited the energy savings. As the PV systems and smart inverter locations were randomly assigned, it's possible that these nodes did not have PV in any of the PV penetration scenarios. If PV had been targeted at these locations, with or without a smart inverter, the voltage at this node would have increased on average. The resulting rise in voltage, at this specific location, potentially would have allowed for the LTC to tap lower, thus allowing for energy savings across the distribution system.

- System base voltage level. While the PG\&E distribution system operated on a $21 \mathrm{kV}$ voltage base, the HECO system operated on a $12 \mathrm{kV}$ voltage base. The higher voltage likely contributed to higher energy savings for PG\&E's distribution system compared to HECO's. This phenomenon was noted in PNNL's "Evaluation of Conservation Voltage Reduction on a National Level", as the study demonstrated that energy savings from voltage reduction were higher on "heavily loaded, higher voltage feeders" [5].

Another interesting insight can come out of this distribution system characteristic. While the highest voltage reduction energy savings resulted with the higher distribution voltage, the highest energy savings improvement with PV and smart inverters resulted with the lower distribution voltage. At the time of PNNL's study, smart inverter volt-VAR control was not considered as an option to augment voltage reduction programs. If smart inverters were included in future studies, the results could be significantly different as 
smart inverters have the potential to unlock increased voltage reduction energy savings not previously possible without traditional mitigation.

\subsection{Photovoltaic Curtailment}

Given that the inverters were sized smaller than the PV system and that the smart inverter's VVC was simulated with VAR priority, the amount of active power curtailment could be a concern. Table 28 and Table 29 show the generation curtailment percentage for VO for the PG\&E and HECO distribution systems respectively. For the same PV penetration level, there is a slight increase in the amount of curtailment among different smart inverter densities. The percentage of PV curtailment is less than $0.7 \%$ for all scenarios in the PG\&E system model and less than $0.3 \%$ for all scenarios with smart inverter density less than 50\%. The percentage of PV curtailment is less than $1.3 \%$ for all scenarios in the HECO system model. Compared with the extra voltage reduction energy savings achieved, the PV curtailment is relatively small.

Table 28. Percentage of PV Curtailment with CVR VO for the PG\&E Distribution System

\begin{tabular}{c|c|c|c|c|c}
\hline \multicolumn{3}{c|}{\begin{tabular}{c} 
PV Active Power \\
\multicolumn{2}{c}{ Curtailment (\%) }
\end{tabular}} & \multicolumn{4}{|c}{ Smart Inverter Density (\%) } \\
\cline { 2 - 6 } & $\mathbf{0}$ & $\mathbf{2 5}$ & $\mathbf{5 0}$ & $\mathbf{1 0 0}$ \\
\hline \multirow{4}{*}{$\begin{array}{c}\text { PV } \\
\begin{array}{c}\text { Petration } \\
(\%)\end{array}\end{array}$} & $\mathbf{5}$ & 0.0 & & & \\
\cline { 2 - 6 } & $\mathbf{1 0}$ & 0.0 & 0.1 & 0.3 & 0.7 \\
\cline { 2 - 6 } & $\mathbf{2 0}$ & 0.0 & 0.2 & 0.3 & 0.5 \\
\cline { 2 - 6 } & $\mathbf{3 0}$ & 0.0 & 0.2 & 0.3 & 0.6 \\
\cline { 2 - 6 } & $\mathbf{5 0}$ & 0.0 & 0.1 & 0.3 & 0.3 \\
\cline { 2 - 6 } & $\mathbf{1 0 0}$ & 0.0 & 0.2 & 0.2 & 0.1 \\
\hline
\end{tabular}

\begin{tabular}{|c|}
\hline Scale \\
\hline 0.0 \\
0.5 \\
1.0 \\
1.5 \\
\hline
\end{tabular}

Table 29. Percentage of PV Curtailment with CVR VO for the HECO Distribution System

\begin{tabular}{c|c|c|c|c|c|c}
\hline \multicolumn{3}{c|}{$\begin{array}{c}\text { PV Active Power } \\
\text { Curtailment (\%) }\end{array}$} & \multicolumn{5}{|c}{ Smart Inverter Density (\%) } \\
\cline { 2 - 7 } & $\mathbf{0}$ & $\mathbf{2 5}$ & $\mathbf{5 0}$ & $\mathbf{7 5}$ & $\mathbf{1 0 0}$ \\
\hline \multirow{4}{*}{\begin{tabular}{c} 
PV \\
\cline { 2 - 7 } \\
\cline { 2 - 7 }
\end{tabular}} & $\mathbf{5 0}$ & 0.00 & & & & \\
\cline { 2 - 7 } & $\mathbf{7 5}$ & 0.00 & 0.42 & 0.67 & 0.78 & 0.92 \\
\cline { 2 - 7 } & $\mathbf{1 0 0}$ & 0.00 & 0.39 & 0.55 & 0.59 & 0.68 \\
\cline { 2 - 7 } & $\mathbf{1 2 5}$ & 0.00 & 0.42 & 0.56 & 0.67 & 0.79 \\
\cline { 2 - 7 } & $\mathbf{1 5 0}$ & 0.00 & 0.24 & 0.23 & 0.25 & 0.68 \\
\hline
\end{tabular}

Scale

0.0

0.5

1.0

1.5 


\section{Conclusion}

This report assessed the impact that distributed PV with smart inverter volt-VAR control can have on voltage reduction energy savings and power quality. A methodology to implement CVR with VO was developed that controls the substation LTC and, when available, switched capacitor banks. Smart inverters participated in the program by using their autonomous volt-VAR control. Voltage reduction energy savings were measured, and a power quality scoring methodology was proposed and utilized to define the relative power quality using a single index or PQS. The CVR VO and PQS methodologies were coded into an open-source software package that can be utilized by utilities and other parties for further study and analysis. The report included two case studies, one each from two distribution systems - HECO and PG\&E - to assess the impact of different PV penetrations and smart inverter densities on voltage reduction energy savings and the PQS. High-level summaries of smart inverter impacts are shown in Table 30 and Table 31. The values shown in these two tables represent the results obtained at two extremes among all various PV and smart inverter penetration scenarios studied: the lowest non-zero PV penetration and non-zero smart inverter density, and the highest PV penetration and highest smart inverter density.

Table 30. Summary of the Voltage Reduction Energy Savings from the HECO and PG\&E Case Studies

\begin{tabular}{|c|c|c|c|c|c|}
\hline Scenario $^{a}$ & Feeder & $\begin{array}{c}\text { PV } \\
\text { Penetration }\end{array}$ & $\begin{array}{c}\text { SI } \\
\text { Density }\end{array}$ & $\begin{array}{c}\text { Voltage } \\
\text { Reduction } \\
\text { Energy } \\
\text { Savings (\%) }\end{array}$ & $\begin{array}{l}\text { Additional Energy Savings } \\
\text { from Smart Inverters }(\%)^{\mathbf{b}}\end{array}$ \\
\hline \multirow{4}{*}{$\begin{array}{c}\text { Highest } \\
\text { PV Penetration and } \\
\text { SI Density }\end{array}$} & \multirow{2}{*}{ HECO } & \multirow{2}{*}{$150 \%$} & $0 \%$ & 1.82 & \multirow{2}{*}{+1.06} \\
\hline & & & $100 \%$ & 2.88 & \\
\hline & \multirow{2}{*}{ PG\&E } & \multirow{2}{*}{$100 \%$} & $0 \%$ & 3.89 & \multirow{2}{*}{+0.41} \\
\hline & & & $100 \%$ & 4.30 & \\
\hline \multirow{4}{*}{$\begin{array}{c}\text { Lowest } \\
\text { PV Penetration and } \\
\text { SI Density }\end{array}$} & \multirow{2}{*}{ HECO } & \multirow{2}{*}{$50 \%$} & $0 \%$ & 1.59 & \multirow{2}{*}{+0.30} \\
\hline & & & $25 \%$ & 1.89 & \\
\hline & \multirow{2}{*}{ PG\&E } & \multirow{2}{*}{$5 \%$} & $0 \%$ & 3.84 & \multirow{2}{*}{+0.12} \\
\hline & & & $25 \%$ & 3.96 & \\
\hline
\end{tabular}

Table 31. Summary of the PQS from the HECO and PG\&E Case Studies without CVR VO

\begin{tabular}{|c|c|c|c|c|c|}
\hline Scenario $^{a}$ & Feeder & $\begin{array}{c}\text { PV } \\
\text { Penetration }\end{array}$ & $\begin{array}{c}\text { SI } \\
\text { Density }\end{array}$ & PQS & $\begin{array}{l}\text { PQS Improvement from } \\
\text { Smart Inverters }\end{array}$ \\
\hline \multirow{4}{*}{$\begin{array}{c}\text { Highest } \\
\text { PV Penetration } \\
\text { and SI Density }\end{array}$} & \multirow{2}{*}{ HECO } & \multirow{2}{*}{$150 \%$} & $0 \%$ & 8.59 & \multirow{2}{*}{+0.10} \\
\hline & & & $100 \%$ & 8.69 & \\
\hline & \multirow{2}{*}{ PG\&E } & \multirow{2}{*}{$100 \%$} & $0 \%$ & 9.13 & \multirow{2}{*}{+0.11} \\
\hline & & & $100 \%$ & 9.24 & \\
\hline \multirow{4}{*}{$\begin{array}{l}\text { Lowest } \\
\text { PV Penetration } \\
\text { and SI Density }\end{array}$} & \multirow{2}{*}{ HECO } & \multirow{2}{*}{$50 \%$} & $0 \%$ & 8.98 & \multirow{2}{*}{+0.26} \\
\hline & & & $100 \%$ & 9.24 & \\
\hline & \multirow{2}{*}{ PG\&E } & \multirow{2}{*}{$5 \%$} & $0 \%$ & 9.25 & \multirow{2}{*}{+0.01} \\
\hline & & & $25 \%$ & 9.26 & \\
\hline
\end{tabular}

a "Highest" and "lowest" refer to the highest and lowest non-zero PV penetration and smart inverter density.

${ }^{b}$ The numbers shown in this column were calculated by taking the difference between the non-SI case $(0 \%)$ and the SI case.

The key findings from this report include: 
1. A VO scheme that flattens and lowers the distribution system voltage profile by controlling a substation LTC tap and switching capacitors can reduce overall energy consumption. Without PV, voltage reduction energy savings of $1.51 \%$ and $3.86 \%$ were achieved for the HECO and PG\&E distribution system models, respectively. ${ }^{3}$ The PG\&E distribution system had higher energy savings, most likely a result of the higher distribution system voltage, urban environment, and the presence of seven capacitors. The capacitors were utilized to help flatten the voltage profile and allow for the voltage regulator to tap further down.

2. In some cases, randomly distributed PV without smart inverters can still increase voltage reduction energy savings. Generally, the HECO distribution system showed an approximately linear correlation in which voltage reduction energy savings increased with increasing PV penetration without smart inverters (Figure 24); however, this was not the case with the PG\&E system. Increased PV penetrations without smart inverters had almost no impact, positive or negative, on voltage reduction energy savings for the PG\&E system (Figure 31). ${ }^{4}$ In the case of PG\&E system, the increased voltage was either insubstantial or mitigated by the control scheme. In cases when randomly distributed PV without smart inverters does not increase voltage reduction energy savings, savings may still be possible if PV installations are targeted at low-voltage. This is an area of future research.

3. Voltage reduction energy savings increased with autonomous smart inverter volt-VAR control (Figure 23 and Figure 30). Smart inverters with a lower VVC band center allowed the tap position of the substation LTC to be further lowered compared to cases without smart inverters. This resulted in a lower distribution system voltage profile and increased voltage reduction energy savings. Compared to the no-PV base case, the HECO system saw a $1.06 \%$ increase in voltage reduction energy savings with $150 \% \mathrm{PV}$ penetration and $100 \%$ smart inverter density. The PG\&E system saw a $0.41 \%$ increase in voltage reduction energy savings with 100\% PV penetration and 100\% smart inverter density.

4. This current implementation of the VO scheme had only one decision lever: lowering the voltages at all loads as much as possible. The best smart inverter VVC was determined based on this rule. As a result, this had the unintended consequence of increasing reactive power demand at the substation as the smart inverters absorbed reactive power to lower the voltage. Future areas of research could include optimizing reactive power as well to determine the potential energy savings of autonomous smart inverter control with voltVAR optimization. One possible way to execute this would be to use the aggregate distribution system's energy usage as a decision lever and appropriately control for the loss reductions made possible by distributed PV without voltage reduction.

\footnotetext{
${ }^{3}$ These savings were based on modeling the loads with a CVR factor of 0.8. If the CVR factor for the load type studied is known to be higher or lower, an approximately linear increase or decrease in savings is expected.

${ }^{4}$ This is interesting because one might expect voltage reduction energy savings to diminish at increased PV penetrations. Voltage may increase downstream when reverse power flow occurs, causing the opposite effect of CVR. This is most likely a result of a combination of factors that not only includes the voltage reduction scheme, but also the way in which the PV was distributed and the secondary system modeled.
} 
5. Because voltage reduction energy savings were prioritized over the PQS, the implementation of the VO scheme lowered certain power quality scoring metrics, including SCDOI and SRPDI, causing an overall lower PQS. Compared to the base case, the combination of VO being active and smart inverter volt-VAR control increased both reactive power demand and regulator tap changes. The increased reactive power demand occurred because the volt-VAR control of the smart inverter absorbs VARs to lower voltage, leading to a lower SRPDI score. With this voltage reduction, more regulator tap changes occurred to lower the voltage and increase the voltage reduction energy savings. While an optimal solution for voltage reduction and PQS was not identified in this study, this report was a result of the present iteration of the voltage reduction implementation, and different results would most likely occur with a different control approach that cooptimizes for regulator tap changes, reactive power demand, and voltage reduction. Also, as previously discussed, the definition of the maximum and minimum value of each individual score can influence the overall PQS. The interpolation of SCDOI from its raw value to its score may have inadvertently given more weight to the SCDOI metric, further lowering the PQS. Future studies should interpolate all components of the PQS by either theoretical maximum limits or more practical limits based on real-world experiences.

6. Overall, without VO, smart inverters had a positive impact on the PQS. In the PG\&E model, there was a PQS increase compared to the base case in almost all smart inverter scenarios studied. In the HECO model with smart inverters, all metrics except SCDOI generally showed a power quality improvement compared to the base case, indicating that distributed PV with smart inverters have the potential to improve power quality. The decrease in the metric for SCDOI could have possibly improved under different LTC settings. The settings used were not optimized for every PV penetration and smart inverter scenario studied. Future studies could run sensitivity analyses on the LTC settings to further demonstrate how smart inverters can improve power quality. 


\section{References}

1. A. Bokhari, et al. "Combined Effect of CVR and DG Penetration in the Voltage Profile of Low-Voltage Secondary Distribution Networks," IEEE Transactions on Power Delivery, 31:1 (Feb. 2016): 286-293.

2. R. Moghe, D. Tholomier, D. Divan, J. Schatz, and D. Lewis, "Grid Edge Control: A New Approach for volt-VAR Optimization" (paper presented at the 2016 IEEE/PES Transmission and Distribution Conference and Exposition [T\&D], Dallas, Texas).

3. S. R. Abate, T. E. McDermott, M. Rylander and J. Smith, "Smart Inverter Settings for Improving Distribution Feeder Performance" (paper presented at the 2015 IEEE Power \& Energy Society General Meeting, Denver, Colorado, July).

4. Electric Power Research Institute, Analysis to Inform CA Grid Integration: Methods and Default Settings to Effectively Use Advanced Inverter Functions in the Distribution System (Technical Report 3002007139) (Palo Alto, CA: 2015).

5. K.P. Schneider, F.K. Tuffner, J.C. Fuller, and R. Singh, Evaluation of Conservation Voltage Reduction (CVR) on a National Level (Technical Report) (Richland, WA: 2010). http://www.pnl.gov/main/publications/external/technical reports/PNNL-19596.pdf.

6. American National Standards Institute, C84.1-2011: American National Standard for Electric Power Systems and Equipment—Voltage Ratings (60 Hertz), 2011.

7. Z. Wang and J. Wang, "Review on Implementation and Assessment of Conservation Voltage Reduction," IEEE Transactions on Power Systems 29:3 (May 2014): 1,306-1,315.

8. Electric Power Research Institute, Green Circuits: Distribution Efficiency Case Studies (Technical Report 1023518) (Palo Alto, CA: 2011).

9. Institute of Electrical and Electronics Engineers, Standard 1547a-2014: IEEE Standard for Interconnecting Distributed Resources with Electric Power Systems-Amendment 1, May 2014.

10. Electric Power Research Institute, Modeling High-Penetration PV for Distribution Interconnection Studies: Smart Inverter Function Modeling in OpenDSS, Rev. 2 (Technical Report 3002002271) (Palo Alto, CA: 2013).

11. "OpenDSS," Electric Power Research Institute. https://sourceforge.net/projects/electricdss/.

12. Institute of Electrical and Electronics Engineers, Standard 1453-2015: IEEE Recommended Practice for the Analysis of Fluctuating Installations on Power Systems, September 2015.

13. Electric Power Research Institute, Stochastic Analysis to Determine Feeder Hosting Capacity for Distributed Solar PV (Technical Report 1026640) (Palo Alto, CA: 2012). 
14. Electric Power Research Institute, Impact of High-Penetration PV on Distribution System Performance (Technical Report 1021982) (Palo Alto, CA: 2011).

15. Institute of Electrical and Electronics Engineers, Standard 1250-2011: IEEE Guide for Identifying and Improving Voltage Quality in Power Systems, March 2011.

16. J. H. Harlow, Electric Power Transformer Engineering (CRC Press LLC: 2004).

17. Institute of Electrical and Electronics Engineers, Standard C57.12.00-2010: IEEE Standard for General Requirements for Liquid-Immersed Distribution, Power, and Regulating Transformers, September 2010.

18. "SynerGi," DNV-GL. https://www.dnvgl.com/services/power-distribution-system-andelectrical-simulation-software-synergi-electric-5005.

19. "CYMDIST," EATON, http://www.cyme.com/software/cymdist/.

20. Conversion Tools: Synergi2DSS, CYME2DSS, GOS2DSS, GIS2DSS, Synergi2GLM and CYME2GLM, Software Record SWR-16-28, National Renewable Energy Laboratory Alliance for Sustainable Energy LLC, Feb. 2016.

21. Global Energy Partners, LLC, Distribution Efficiency Initiative-Market Progress Evaluation Report, No.1 (Technical Report \#E05-139) (Walnut Creek, CA: May 2005).

22. National Rural Electric Cooperative Association, Costs and Benefits of Conservation Voltage Reduction - CVR Warrants Careful Examination, Final Report (Technical Report) (Arlington, VA: May 2014).

23. P. Kundur, Power System Stability and Control (New York: McGraw-Hill, Inc., 1994, pp. 257-263).

24. R. Preiss and V. Warnock, "Impact of Voltage Reduction on Energy and Demand," IEEE Transactions on Power Apparatus and Systems (1978): 1,665-1,671. 